Pure and Applied Mathematics Quarterly

Volume 6, Number 3

(Special Issue: In honor of

Joseph J. Kohn, Part 1 of 2)

915-982, 2010

\title{
Infinitesimal Isospectral Deformations of Symmetric Spaces: Quotients of the Special Unitary Group
}

\author{
Jacques Gasqui and Hubert Goldschmidt \\ To J. J. Kohn on his 75-th birthday
}

\begin{abstract}
We show that the reduced spaces of the special unitary group $S U(n)$ and the symmetric space $S U(2 n) / S p(n)$, with $n \geq 3$, possesses nontrivial infinitesimal isospectral deformations. For the reduced space $X$ of the unitary group $S U(n)$, we also prove a related result: in all degrees $p \geq 2$, there exist symmetric $p$-forms on $X$ which satisfy the Guillemin condition and are not symmetrized covariant derivatives of symmetric $(p-1)$-forms, unless $n=p=3$.
\end{abstract}

Keywords: symmetric space, special unitary group, Radon transform, infinitesimal isospectral deformation, symmetric form, Guillemin condition

\section{INTRODUCTION}

In this paper, we pursue our study of the infinitesimal deformations of the symmetric spaces of compact type, which are both irreducible and reduced, undertaken in [3], [4] and [5]. Motivated by a result due to Guillemin [7], we introduced the space $I(X)$ of infinitesimal isospectral deformations of a Riemannian symmetric space $(X, g)$ of compact type. If $I(X)$ vanishes, we say that $(X, g)$ is rigid in the sense of Guillemin; in this case, we know that every isospectral deformation of the metric $g$ is trivial to first-order, and so the space $X$ is spectrally rigid to first-order.

Received June 1, 2007.

1991 Mathematics Subject Classification. 44A12, 53C35, 58A10, 58J53. 
The homogeneous polynomials of degree 3 on the Lie algebra of the unitary group $S U(n)$ allow us to construct non-trivial infinitesimal isospectral deformations of the reduced Lagrangian Grassmannian, which is a quotient of the symmetric space $S U(n) / S O(n)$. The same method is used here to produce such deformations of a symmetric space belonging to one of the two other classes of irreducible symmetric spaces of compact type, which are irreducible, reduced and quotients of the unitary group, namely the following families, where the integer $n$ is $\geq 3$ :

(i) the reduced space of the special unitary group $G=S U(n)$;

(ii) the reduced space of the symmetric space $S U(2 n) / S p(n)$.

The reduced space of the special unitary group $G=S U(n)$, with $n \geq 3$, viewed as a symmetric space is the quotient group $G / S$, where $S$ is the center of $G$. The latter group is isomorphic to the adjoint group of $\mathfrak{s u}(n)$ and is called the reduced unitary group. The reduced space of the symmetric space $X=S U(2 n) / S p(n)$, with $n \geq 3$, is the quotient of $X$ by the action of the center of the group $S U(2 n)$ on $X$.

As in [3], we say that a symmetric $p$-form $u$ on a symmetric space $(X, g)$ satisfies the Guillemin condition if, for every maximal flat totally geodesic torus $Z$ contained in $X$ and for all parallel vector fields $\zeta$ on $Z$, the integral

$$
\int_{Z} u(\zeta, \zeta, \ldots, \zeta) d Z
$$

vanishes, where $d Z$ is the Riemannian measure of $Z$. The kernel $\mathcal{N}_{p}$ of the Radon transform for $p$-forms consists precisely of those forms satisfying the Guillemin condition.

Let $\left\{g_{t}\right\}$ be a family of Riemannian metrics on $X$, with $g_{0}=g$; assume that $\left\{g_{t}\right\}$ is an isospectral deformation of $g$ (i.e., that the spectrum of the Laplacian of the metric $g_{t}$ is independent of $t$ ). Guillemin proved, using the methods he introduced in [7], that the corresponding infinitesimal deformation $h=\frac{d}{d t} g_{t \mid t=0}$ of the metric $g$ belongs to the kernel $\mathcal{N}_{2}$. If $\varphi_{t}$ is a one-parameter family of diffeomorphisms of $X$, the family $\left\{\varphi_{t}^{*} g\right\}$ is a trivial isospectral deformation; in fact, the space $\mathcal{L}_{2}$ of Lie derivatives of the metric $g$ is a subspace of $\mathcal{N}_{2}$. This leads us to define the space $I(X)$ of infinitesimal isospectral deformations as the 
orthogonal complement of $\mathcal{L}_{2}$ in $\mathcal{N}_{2}$. Thus we have the orthogonal decomposition

$$
\mathcal{N}_{2}=\mathcal{L}_{2} \oplus I(X),
$$

and we denote by $P$ the orthogonal projection of $\mathcal{N}_{2}$ onto $I(X)$. If $I(X)$ vanishes, the infinitesimal deformation $h$ is a Lie derivative of the metric and the deformation $\left\{g_{t}\right\}$ is trivial to first-order.

Let $X$ be a symmetric space belonging to one of the families (i) or (ii). Its universal cover is the symmetric space corresponding to the Riemannian symmetric pair $(\tilde{G}, K)$, which is either $\left(G \times G, G^{*}\right)$ or $(G, S p(n))$, where $G=S U(n)$ for the first pair and $G=S U(2 n)$ for the second one, with $n \geq 3$, and where $G^{*}$ is the diagonal of $G \times G$. For $p \geq 2$, the symmetric space $X$ carries a natural symmetric $\tilde{G}$-invariant $p$-form $\sigma_{p}$ which is induced by the $G$-invariant homogeneous polynomial $Q_{p}$ on the Lie algebra $\mathfrak{g}_{0}$ of $G$ defined by

$$
Q_{p}(A)=(-i)^{p} \operatorname{Tr} A^{p},
$$

for all $A \in \mathfrak{g}_{0}$. In fact, the form $\sigma_{2}$ is equal to the Riemannian metric $g$ of $X$ and the form $\sigma_{3}$ is up to a constant the only $\tilde{G}$-invariant symmetric 3 -form on $X$ (see $[4, \S 2])$.

The form $\sigma_{p}$ induces an injective mapping $\tilde{\sigma}_{p}$ from the space of 1-forms on $X$ to the space of symmetric $(p-1)$-forms on $X$. We show that a 1-form $\theta$ on $X$ satisfies the Guillemin condition if and only if the symmetric $(p-1)$-form $\tilde{\sigma}_{p}(\theta)$ satisfies the Guillemin condition. In particular, the mapping $\tilde{\sigma}_{3}$ sends the space of 1-forms on $X$ into the space of symmetric 2-forms on $X$. We consider the $G$-module $C_{\mathbb{R}}^{\infty}(X)$ of real-valued functions on $X$; if $f$ is an element of $C_{\mathbb{R}}^{\infty}(X)$, the symmetric 2 -form $\tilde{\sigma}_{3}(d f)$ satisfies the Guillemin condition. We prove that the space $\mathcal{F}_{X}^{\prime}$ of functions $f \in C_{\mathbb{R}}^{\infty}(X)$ for which the symmetric 2-form $\tilde{\sigma}_{3}(d f)$ is a Lie derivative of the metric $g$ is the sum of two irreducible $G$-submodules of $C_{\mathbb{R}}^{\infty}(X)$, one of which is the space of constant functions. Thus if $\mathcal{F}_{X}$ is the orthogonal complement of $\mathcal{F}_{X}^{\prime}$ in $C_{\mathbb{R}}^{\infty}(X)$, the sum

$$
\mathcal{L}_{2} \oplus \tilde{\sigma}_{3}\left(d \mathcal{F}_{X}\right)
$$

is direct. The mapping

$$
P \cdot \tilde{\sigma}_{3} \cdot d: \mathcal{F}_{X} \rightarrow I(X)
$$

is therefore injective, and we have thus constructed an explicit infinite-dimensional subspace of $I(X)$. 
Here we also examine a generalization of our problem, which is not related to isospectral deformations and involves symmetric forms of arbitrary degree on a reduced symmetric space of compact type $X$. A symmetric $p$-form on $X$ which is the symmetrized covariant derivative of a symmetric $(p-1)$-form satisfies the Guillemin condition. Verifying that the only symmetric $p$-forms which satisfy the Guillemin condition are precisely the symmetrized covariant derivatives of symmetric $(p-1)$-forms is an injectivity question for Radon transforms which generalizes the Guillemin rigidity problem for $X$. Indeed, for $p=2$, a positive answer to this question is equivalent to the Guillemin rigidity of $X$. For the real projective spaces, this verification was carried out in all degrees (see $\S 3$, Chapter III of [3]). In [3] and [4], we showed that the answer to this question is also positive for 1-forms on the reduced space of an irreducible Grassmannian and on the reduced space of the Lagrangian Grassmannian $S U(4) / S O(4)$.

We show that the answer to this question is negative for the reduced space $X=S U(n) / S$, with $n \geq 3$, in all degrees $p \geq 2$ unless $n=p=3$. We introduce explicit subspaces $\mathcal{F}_{1}$ and $\mathcal{F}_{2}$ of the space of real-valued functions on $X$ which are infinite-dimensional and orthogonal to the subspace of constant functions. Let $p \geq 2$ be a given integer and suppose that $(n, p) \neq(3,3)$; if $p$ is even (resp. odd) and $f$ is an element of the space $\mathcal{F}_{1}$ (resp. the space $\mathcal{F}_{2}$ ), the symmetric $p$-form $\tilde{\sigma}_{p+1}(d f)$, which satisfies the Guillemin condition, is not the symmetrized covariant derivative of a symmetric $(p-1)$-form. When $p=2$, we recover the fact that the space $I(X)$ does not vanish; in fact, the space $\mathcal{F}_{1}$ is a subspace of $\mathcal{F}_{X}$. When $n=3$, the symmetric form $\sigma_{4}$ is a multiple of the symmetric product $g \cdot g$; thus in this case, if $f$ is an arbitrary function on $X$, it is easily seen that the 3 -form $\tilde{\sigma}_{4}(d f)$ is a multiple of the symmetrized covariant derivative of the 2 -form $f g$. Therefore when $n=p=3$, our methods do not give an answer to our question. It is precisely for this reason that we are obliged to distinguish between the cases when $p$ is even or odd.

The harmonic analysis of the special unitary group plays an important role in the proofs of all our results. As above we view the symmetric space $G=S U(n)$ as a homogeneous space of the product $\tilde{G}=G \times G$ by its diagonal subgroup $G^{*}$. We endow the space $C^{\infty}(G)$ of complex-valued functions on $G$ with the $\tilde{G}$-module structure induced by the action of $\tilde{G}$ on its homogeneous space $G$ and with the $G$-module structure induced by the left action of $G$ on itself. We determine the highest weight vectors of certain isotypic components of $C^{\infty}(G)$ with respect to 
its two module structures and also find expressions for the highest weight vectors of certain isotypic components of the space of 1 -forms viewed as a $\tilde{G}$-module. These descriptions are one of the main ingredients of the proofs of our results for the spaces of the family (i).

The proofs of our results concerning the spaces of the family (ii) follow the same lines as those found in [5] for the Lagrangian Grassmannians. However some differences arise from the fact that the rank of $X$ is not equal to the rank of $G$.

We now briefly describe the contents of the various sections of this paper. Chapter I is devoted to all our results concerning the special unitary group $G=S U(n)$ and the symmetric space $X$ which is equal to the reduced space of $G$. In $\S 1$, we introduce objects, notation and terminology associated with Riemannian manifolds and symmetric spaces. In $\S 2$, we present properties of the mapping $\tilde{\sigma}_{p}^{\prime}$, induced by the invariant polynomial $Q_{p}$ on the Lie algebra of $G$, from the space of 1 -forms to the space of symmetric $(p-1)$-forms on $G$. In $\S 3$, we define the explicit functions on $G$ which are required for our results on the harmonic analysis of the group $G$; Lemma 3.2 provides us with a crucial relation involving the mapping $\tilde{\sigma}_{3}$ and some of these functions. In $\S 4$, we undertake a study of the harmonic analysis of the group $G$ and, in particular, of the isotypic components of the space $C^{\infty}(G)$ of functions and the space of complex symmetric $p$-forms on $G$. An analysis of the isotypic components of the space of 1-forms on $G$ and their weight spaces allows us to demonstrate Lemmas 5.5 and 5.7; on the other hand, Lemma 3.2 gives us Lemma 5.6. The proofs of our main results (Propositions 6.1-6.3) concerning symmetric $p$-forms on the group $G$ require both Proposition 8.1 of [5] and Lemmas 5.6, 5.7 and 6.5. The main results concerning isospectral deformations and symmetric $p$-forms on $X$ described above (Theorems 7.4 and 7.5) are consequences of these propositions.

In Chapter II, we study the symmetric space $Y=S U(2 n) / S p(n)$ and its reduced space. In $\S 8$, we present a branching law and the decomposition of a space of tensors into irreducible $S p(n)$-modules. In $\S 9$, we examine properties of the mapping $\tilde{\sigma}_{p}$, induced by the polynomial $Q_{p}$ on the Lie algebra of the group $G=S U(2 n)$, from the space of 1 -forms to the space of symmetric $(p-1)$-forms on $Y$; here, we introduce an explicit maximal flat totally geodesic torus of this symmetric space. In $\S 10$, we define $S p(n)$-invariant functions on the group $G$ 
which give rise to highest weight vectors of the space $C^{\infty}(Y)$ of functions on $Y$ viewed as a $G$-module. The proofs of our results concerning $Y$ and its reduced space are carried out in $\S \S 11-12$ in a way which is completely parallel to the one used in [5] to prove the analogous results for the special Lagrangian Grassmannian $S U(n) / S O(n)$ and its reduced space. Lemmas 11.2-11.4 are crucial ingredients for our proof of the non-triviality of the space of infinitesimal isospectral deformations of the reduced space of $Y$ (Theorem 12.2); we note that the proof of Lemma 11.2 requires Proposition 8.1 of [5].

This paper may be considered as the continuation of [5]. It can be read independently of [5], although, as we have seen above, we use certain results from that paper.

\section{Contents}

Introduction 915

$\begin{array}{ll}\text { CHAPTER I: The special unitary group } & 921\end{array}$

1. Riemannian manifolds 921

2. The special unitary group $\quad 924$

3. Functions on the special unitary group $\quad 929$

4. Highest weight vectors and functions on $S U(n) \quad 933$

5. Highest weight vectors and forms of degree one on $S U(n) \quad 939$

6. Isospectral deformations of the special unitary group 943

7. The reduced unitary group $\quad 950$

CHAPTER II: The symmetric space $S U(2 n) / S p(n) \quad 953$

8. Branching laws and the decomposition of a space of tensors 953

9. The symmetric space $S U(2 n) / S p(n) \quad 960$

10. Functions on the symmetric space $S U(2 n) / S p(n) \quad 966$

11. Isospectral deformations of $S U(2 n) / S p(n) \quad 972$

12. The reduced space of $S U(2 n) / S p(n) \quad 979$

$\begin{array}{ll}\text { References } & 982\end{array}$ 


\section{CHAPTER I: THE SPECIAL UNITARY GROUP}

\section{Riemannian MANiFOLDS}

Let $X$ be a differentiable manifold, whose tangent and cotangent bundles we denote by $T=T_{X}$ and $T^{*}=T_{X}^{*}$, respectively. Let $C^{\infty}(X)\left(\operatorname{resp} . C_{\mathbb{R}}^{\infty}(X)\right)$ be the space of complex-valued (resp. real-valued) functions on $X$. Let $\mathbb{R}(X)$ denote the subspace of $C_{\mathbb{R}}^{\infty}(X)$ consisting of the constant functions on $X$. Let $E$ be a vector bundle over $X$; we denote by $E_{\mathbb{C}}$ its complexification, by $\mathcal{E}$ the sheaf of sections of $E$ over $X$ and by $C^{\infty}(E)$ the space of global sections of $E$ over $X$. By $\otimes^{k} E, S^{l} E$ and $\bigwedge^{j} E$, we shall mean the $k$-th tensor product, the $l$-th symmetric product and the $j$-th exterior product of the vector bundle $E$, respectively. We shall identify $S^{k} T^{*}$ and $\bigwedge^{k} T^{*}$ with sub-bundles of $\otimes^{k} T^{*}$ as in $\S 1$, Chapter I of [3]. In particular, if $\alpha, \beta \in T^{*}$, the symmetric product $\alpha \cdot \beta$ is identified with the element $\alpha \otimes \beta+\beta \otimes \alpha$ of $\otimes^{2} T^{*}$. If $u$ is a section of $S^{p} T^{*}$ over $X$, we consider the morphism of vector bundles

$$
u^{b}: T \rightarrow S^{p-1} T^{*}
$$

defined by

$$
\left(u^{b} \xi\right)\left(\eta_{1}, \ldots, \eta_{p-1}\right)=u\left(\xi, \eta_{1}, \ldots, \eta_{p-1}\right),
$$

for $\xi, \eta_{1}, \ldots, \eta_{p-1} \in T$.

Let $g$ be a Riemannian metric on $X$. We denote by $g^{\sharp}: T^{*} \rightarrow T$ the inverse of the isomorphism $g^{b}: T \rightarrow T^{*}$. If $u$ is a section of $S^{p} T^{*}$ over $X$, we consider the morphism of vector bundles

$$
\tilde{u}=u^{b} \cdot g^{\sharp}: T^{*} \rightarrow S^{p-1} T^{*} .
$$

We also consider the scalar products on the spaces of sections $C^{\infty}(X), C^{\infty}(T)$ and $C^{\infty}\left(S^{2} T^{*}\right)$, defined in terms of the Riemannian measure of $X$ and the scalar products on the vector bundles $T$ and $S^{2} T^{*}$ induced by the metric $g$. We denote by $C_{\mathbb{R}, 0}^{\infty}(X)$ the orthogonal complement of the subspace $\mathbb{R}(X)$ of $C_{\mathbb{R}}^{\infty}(X)$.

Let $\nabla$ be the Levi-Civita connection of $(X, g)$. For $p \geq 0$, we consider the symmetrized covariant derivative

$$
D^{p}: S^{p} \mathcal{T}^{*} \rightarrow S^{p+1} \mathcal{T}^{*}
$$


which is the first-order differential operator defined by

$$
\left(D^{p} u\right)\left(\xi_{1}, \ldots, \xi_{p+1}\right)=\frac{1}{p+1} \sum_{j=1}^{p+1}(\nabla u)\left(\xi_{j}, \xi_{1}, \ldots, \hat{\xi}_{j}, \ldots, \xi_{p+1}\right),
$$

for $u \in S^{p} \mathcal{T}^{*}$ and $\xi_{1}, \ldots, \xi_{p+1} \in T$. If $p \geq 1$ and $\omega_{1}, \ldots, \omega_{p}$ are elements of $\mathcal{T}^{*}$ satisfying $D^{1} \omega_{j}=0$, for $1 \leq j \leq p$, we easily verify that

$$
D^{p}\left(\omega_{1} \cdot \ldots \cdot \omega_{p}\right)=0 .
$$

The operator $D^{0}$ is equal to the exterior differential operator $d$ on functions. The Hessian Hess $f$ of a real-valued function $f$ on $X$ is equal to $D^{1} d f=\nabla d f$. On the other hand, the Killing operator

$$
D_{0}: \mathcal{T} \rightarrow S^{2} \mathcal{T}^{*}
$$

of $(X, g)$, which sends a vector field $\xi$ into the Lie derivative $\mathcal{L}_{\xi} g$ of $g$ along $\xi$, and the operator $D^{1}$ are related by the formula

$$
\frac{1}{2} D_{0} \xi=D^{1} g^{b}(\xi)
$$

for $\xi \in \mathcal{T}$. We easily see that

$$
D^{p}(f u)=\frac{1}{p+1} d f \cdot u+f D^{p} u
$$

for all $f \in C_{\mathbb{R}}^{\infty}(X)$ and $u \in C^{\infty}\left(S^{p} T^{*}\right)$. We consider the symmetric 4-form $\sigma=g \cdot g$ on $X$ and the morphism $\tilde{\sigma}: T^{*} \rightarrow S^{3} T^{*}$ which it determines; we have

$$
\frac{1}{2} \sigma\left(\xi_{1}, \xi_{2}, \xi_{3}, \xi_{4}\right)=g\left(\xi_{1}, \xi_{2}\right) g\left(\xi_{3}, \xi_{4}\right)+g\left(\xi_{1}, \xi_{3}\right) g\left(\xi_{2}, \xi_{4}\right)+g\left(\xi_{1}, \xi_{4}\right) g\left(\xi_{2}, \xi_{3}\right),
$$

for all $\xi_{1}, \xi_{2}, \xi_{3}, \xi_{4} \in T$. Hence for all $\varphi \in C^{\infty}\left(T^{*}\right)$, we obtain

$$
\tilde{\sigma}(\varphi)=2 \varphi \cdot g
$$

then for all $f \in C_{\mathbb{R}}^{\infty}(X)$, by means of formula (1.3) we easily see that

$$
6 D^{2}(f g)=\tilde{\sigma}(d f) .
$$

We also consider the divergence operator

$$
\operatorname{div}: S^{2} \mathcal{T}^{*} \rightarrow \mathcal{T}^{*},
$$

as defined in $\S 1$, Chapter I of [3]; we recall that the formal adjoint of $D_{0}$ is equal to $2 g^{\sharp} \cdot \operatorname{div}: S^{2} \mathcal{T}^{*} \rightarrow \mathcal{T}$. When $X$ is compact, since the operator $D_{0}$ is elliptic, we therefore have the orthogonal decomposition

$$
C^{\infty}\left(S^{2} T^{*}\right)=D_{0} C^{\infty}(T) \oplus\left\{h \in C^{\infty}\left(S^{2} T^{*}\right) \mid \operatorname{div} h=0\right\}
$$


given by the relation (1.11) of [3]; we denote by

$$
P: C^{\infty}\left(S^{2} T^{*}\right) \rightarrow\left\{h \in C^{\infty}\left(S^{2} T^{*}\right) \mid \operatorname{div} h=0\right\}
$$

the projection determined by the decomposition (1.5).

We now suppose that $X$ is a symmetric space of compact type. We know that there is a Riemannian symmetric pair $(G, K)$ of compact type, where $G$ is a compact, semi-simple Lie group and $K$ is a closed subgroup of $G$, such that the space $X$ is isometric to the homogeneous space $G / K$ endowed with a $G$-invariant metric. We shall identify $X$ with $G / K$. The spaces $C_{\mathbb{R}}^{\infty}(X)$ and $C^{\infty}(X)$ and the spaces $C^{\infty}\left(S^{p} T^{*}\right)$ and $C^{\infty}\left(S^{p} T_{\mathbb{C}}^{*}\right)$ of symmetric $p$-forms on $X$ inherit structures of $G$-modules from the action of $G$ on $X$. Let $\Gamma$ denote the dual of the group $G$, that is, the set of equivalence classes of irreducible $G$-modules over $\mathbb{C}$ and let $F$ be a $G$-invariant complex sub-bundle of $S^{p} T_{\mathbb{C}}^{*}$. If $\gamma$ is an element of $\Gamma$, we denote by $C_{\gamma}^{\infty}(X)$ and $C_{\gamma}^{\infty}(F)$ the isotypic components of the $G$-modules $C^{\infty}(X)$ and $C^{\infty}(F)$, respectively, corresponding to $\gamma$.

We say that a symmetric $p$-form $u$ on $X$ satisfies the Guillemin condition if, for every maximal flat totally geodesic torus $Z$ contained in $X$ and for all parallel vector fields $\zeta$ on $Z$, the integral

$$
\int_{Z} u(\zeta, \zeta, \ldots, \zeta) d Z
$$

vanishes, where $d Z$ is the Riemannian measure of $Z$. For $p \geq 0$, we consider the $G$-submodule $\mathcal{N}_{p}$ of $C^{\infty}\left(S^{p} T^{*}\right)$ consisting of all symmetric $p$-forms satisfying the Guillemin condition; we recall that $D_{0} C^{\infty}(T)$ is a $G$-submodule of $\mathcal{N}_{2}$ and, more generally, we have

$$
D^{p-1} C^{\infty}\left(S^{p-1} T^{*}\right) \subset \mathcal{N}_{p},
$$

for $p \geq 1$ (see Lemma 2.10 of [3]). In this paper, we are interested in knowing when equality holds in the above inclusion, i.e., when we have the equality

$$
D^{p-1} C^{\infty}\left(S^{p-1} T^{*}\right)=\mathcal{N}_{p} .
$$

We define the space of infinitesimal isospectral deformations of $g$ by

$$
I(X)=\left\{h \in \mathcal{N}_{2} \mid \operatorname{div} h=0\right\} .
$$

From the decomposition (1.5), we obtain the orthogonal decomposition

$$
\mathcal{N}_{2}=D_{0} C^{\infty}(T) \oplus I(X) ;
$$


moreover, the orthogonal projection of $\mathcal{N}_{2}$ onto $I(X)$ is equal to the restriction of the projection $P$ to $\mathcal{N}_{2}$. Thus the vanishing of the space $I(X)$ is equivalent to the fact that the space $X$ is rigid in the sense of Guillemin; by (1.2), we see that it is also equivalent to the fact that the equality (1.6) holds for $p=2$. Moreover if there exists a symmetric 2-form on $X$ belonging to $\mathcal{N}_{2}$ which is not equal to a Lie derivative of the metric $g$, the space $I(X)$ does not vanish.

Let $\sigma$ be a $G$-invariant symmetric $(p+1)$-form on $X$, where $p$ is an integer $\geq 2$; clearly, $\sigma$ is parallel and so we have $\nabla \sigma=0$. The morphisms

$$
\sigma^{b}: T \rightarrow S^{p} T^{*}, \quad \tilde{\sigma}: T^{*} \rightarrow S^{p} T^{*}
$$

induced by $\sigma$ are $G$-equivariant; if $X$ is irreducible and $\sigma$ is non-zero, they are monomorphisms of vector bundles.

Assume moreover that the following is true: if a 1-form $\varphi$ on $X$ satisfies the Guillemin condition, the symmetric $p$-form $\tilde{\sigma}(\varphi)$ also satisfies the Guillemin condition. Then if $f$ is an element of $C_{\mathbb{R}}^{\infty}(X)$, the symmetric $p$-form $\tilde{\sigma}(d f)$ satisfies the Guillemin condition. If there exists a non-zero subspace $\mathcal{F}$ of $C_{\mathbb{R}, 0}^{\infty}(X)$ such that the relation

$$
D^{p-1} C^{\infty}\left(S^{p-1} T^{*}\right) \cap \tilde{\sigma} d \mathcal{F}=\{0\}
$$

holds, then

$$
D^{p-1} C^{\infty}\left(S^{p-1} T^{*}\right) \oplus \tilde{\sigma} d \mathcal{F}
$$

is a subspace of $\mathcal{N}_{p}$ and the equality (1.6) does not hold.

We now suppose that $p=2$. If $P$ is the orthogonal projection corresponding to the decomposition (1.5), the mapping

$$
P_{\sigma}: P \tilde{\sigma} d: C_{\mathbb{R}}^{\infty}(X) \rightarrow I(X)
$$

is well-defined. Clearly, if $f$ is an element of $C_{\mathbb{R}}^{\infty}(X)$, then $\tilde{\sigma} d f$ is a Lie derivative of the metric if and only if $P_{\sigma} f=0$.

\section{The SPECIAL Unitary GRouP}

Let $n \geq 3$ be a given integer. Let $X=G$ be the special unitary group $S U(n)$. If $B$ denotes the Killing form of the Lie algebra $\mathfrak{g}_{0}=\mathfrak{s u}(n)$, we endow $X$ with the bi-invariant Riemannian metric $g_{0}$ induced by $-B$. As usual, we identify the $G$-module $\mathfrak{g}_{0}$ with the tangent space of $X$ at the identity element $e_{0}=I_{n}$ of $G$. We consider the involutive automorphism $s$ of the group $\tilde{G}=G \times G$ which 
sends $\left(g_{1}, g_{2}\right)$ into $\left(g_{2}, g_{1}\right)$. The fixed point set of $s$ is the diagonal subgroup $G^{*}$ of $G \times G$; thus the pair $\left(\tilde{G}, G^{*}\right)$ is a Riemannian symmetric pair. Since the homogeneous space $\tilde{G} / G^{*}$ is diffeomorphic to the group $G$ under the mapping $\tilde{G} / G^{*} \rightarrow G$, sending $\left(g_{1}, g_{2}\right) G^{*}$ into $g_{1} g_{2}^{-1}$, where $g_{1}, g_{2} \in G$, we may identify $X$ with the homogeneous space $\tilde{G} / G^{*}$. Then the action of the group $\tilde{G}$ on the space $X$ is given by

$$
\left(g_{1}, g_{2}\right) \cdot a=g_{1} a g_{2}^{-1}
$$

for all $g_{1}, g_{2}, a \in G$; it induces $\tilde{G}$-module structures on the spaces $C^{\infty}(G)$ and $C^{\infty}\left(S^{p} T_{\mathbb{C}}^{*}\right)$. A symmetric form on $X$ is $\tilde{G}$-invariant if and only if it is bi-invariant under the action of $G$. Thus the metric $g_{0}$ on $X$ is $\tilde{G}$-invariant and the manifold $X$ endowed with this metric is an irreducible symmetric space.

If $k \geq 1$ is a given integer, we consider the space $M_{k}$ of all $k \times k$ complex matrices. For $1 \leq j, k \leq n$, let $E_{j k}=\left(c_{l r}\right)$ be the element of $M_{n}$ determined by $c_{j k}=1$ and $c_{l r}=0$ whenever $(l, r) \neq(j, k)$. If $1 \leq j, k \leq n$ and $1 \leq l \leq n-1$ are integers, with $j \neq k$, the matrices

$$
A_{j k}=E_{j k}-E_{k j}, \quad B_{j k}=i\left(E_{j k}+E_{k j}\right), \quad C_{l}=i\left(E_{l l}-E_{l+1, l+1}\right)
$$

of $M_{n}$ belong to $\mathfrak{g}_{0}$; in fact, the set of all these matrices $\left\{A_{j k}, B_{j k}, C_{l}\right\}$, with $1 \leq j<k \leq n$ and $1 \leq l \leq n-1$, form a basis of $\mathfrak{g}_{0}$. For $1 \leq j \leq n$, we consider the element

$$
\tilde{C}_{j}=\frac{1}{n}\left(\sum_{k=j}^{n-1}(n-k) C_{k}-\sum_{k=1}^{j-1} k C_{k}\right)
$$

of $\mathfrak{g}_{0}$.

For $p \geq 2$, the homogeneous polynomial $Q_{p}$ on $\mathfrak{g}_{0}$ defined by

$$
Q_{p}(\xi)=(-i)^{p} \operatorname{Tr} \xi^{p},
$$

for all $\xi \in \mathfrak{g}_{0}$, is $G$-invariant, non-zero and real-valued; therefore it gives rise to a non-zero bi-invariant symmetric $p$-form $\sigma_{p}^{\prime}$ on $X$. It is well-known that the algebra of all $G$-invariant polynomials on $\mathfrak{g}_{0}$ is generated by the polynomials $Q_{p}$, with $2 \leq p \leq n$, and that these polynomials are algebraically independent. We know that the metric $g_{0}$ is equal to the symmetric 2 -form $2 n \cdot \sigma_{2}^{\prime}$ and that $\sigma_{3}^{\prime}$ is up to a constant the unique bi-invariant symmetric 3 -form on $X$ (see $[4, \S 2]$ ).

We shall always consider the symmetric space $X=S U(n)$, with $n \geq 3$, endowed with the Riemannian metric $g^{\prime}=\sigma_{2}^{\prime}$. We easily verify that the product of 
matrices $C_{j} \cdot C_{k}$ is equal to 0 , for all $1 \leq j, k \leq n-1$, with $j<k+1$, and hence that

$$
g^{\prime}\left(C_{j}, C_{j}\right)=2, \quad g^{\prime}\left(C_{l}, C_{l+1}\right)=-1, \quad g^{\prime}\left(C_{k}, C_{q}\right)=0,
$$

for all $1 \leq j, k, q \leq n-1$ and $1 \leq l \leq n-2$, with $q \geq k+2$.

We identify an element of $\mathfrak{g}_{0}$ with the left-invariant vector field on $G$ that it determines. Throughout the remainder of this section, by $C_{j}, \tilde{C}_{l}, A_{k l}$ and $B_{k l}$ we shall often mean the left-invariant vector fields on $G$ determined by the corresponding elements of $\mathfrak{g}_{0}$. Let $\left\{\omega_{0}, \omega_{j}, \omega_{j k}, \varpi_{j k}\right\}$, with $1 \leq j, k \leq n$, be the left-invariant 1-forms on $G$ determined by

$$
\omega_{0}=\omega_{n}=\varpi_{j j}=0, \quad \omega_{j k}=-\omega_{k j}, \quad \varpi_{j k}=\varpi_{k j},
$$

for $1 \leq j, k \leq n$, and

$$
\begin{aligned}
& \omega_{j}\left(C_{l}\right)=\delta_{j l}, \quad \omega_{j k}\left(C_{l}\right)=0, \quad \varpi_{j k}\left(C_{l}\right)=0, \\
& \omega_{j}\left(A_{r s}\right)=0, \quad \omega_{j k}\left(A_{r s}\right)=\delta_{j r} \delta_{k s}, \quad \varpi_{j k}\left(A_{r s}\right)=0, \\
& \omega_{j}\left(B_{r s}\right)=0, \quad \omega_{j k}\left(B_{r s}\right)=0, \quad \varpi_{j k}\left(B_{r s}\right)=\delta_{j r} \delta_{k s},
\end{aligned}
$$

for all $1 \leq l \leq n-1$ and $1 \leq j, k, r, s \leq n$, with $j<k$ and $r<s$. Then $\left\{\omega_{l}, \omega_{j k}, \varpi_{j k}\right\}$, with $1 \leq l \leq n-1$ and $1 \leq j<k \leq n$, is a basis of the space $\mathcal{A}$ of left-invariant 1 -forms on $G$. If $p$ is an integer $\geq 1$, we view the $p$-th symmetric power $S^{p} \mathcal{A}$ of $\mathcal{A}$ as a subspace of $C^{\infty}\left(S^{p} T^{*}\right)$. Since the left-invariant vector fields on $G$ are Killing vector fields, by (1.2) we know that an arbitrary element $\omega$ of $\mathcal{A}$ satisfies $D^{1} \omega=0$. According to the relation (1.1), an element $u$ of $S^{p} \mathcal{A}$ satisfies $D^{p} u=0$. Therefore, if $f$ is a real-valued function on $G$ and $u$ is an element of $S^{p} \mathcal{A}$, by (1.3) we see that

$$
(p+1) D^{p}(f u)=d f \cdot u .
$$

For $p \geq 3$, we consider the monomorphism

$$
\tilde{\sigma}_{p}^{\prime}: T^{*} \rightarrow S^{p-1} T^{*}
$$

induced by the symmetric $p$-form $\sigma_{p}^{\prime}$. We shall write $\sigma^{\prime}=\sigma_{3}^{\prime}$ and $\tilde{\sigma}^{\prime}=\tilde{\sigma}_{3}^{\prime}$. According to $[5, \S 3]$, if $\varphi$ is an element of $C^{\infty}\left(T^{*}\right)$, we know that

$$
\tilde{\sigma}_{p}^{\prime}(\varphi)\left(C_{j_{1}}, C_{j_{2}}, \ldots, C_{j_{p-1}}\right)=0
$$

for $1 \leq j_{1}, j_{2}, \ldots, j_{p-1} \leq n-1$, with $j_{1}>j_{2}+1$, and that

$$
\tilde{\sigma}_{p}^{\prime}(\varphi)\left(C_{j}, \ldots, C_{j}\right)=\varphi\left(\tilde{C}_{j}\right)+(-1)^{p+1} \varphi\left(\tilde{C}_{j+1}\right),
$$


for all $1 \leq j \leq n-1$; moreover, for all $1 \leq j \leq n-2$ and $1 \leq k \leq p-1$, we have

$$
\tilde{\sigma}_{p}^{\prime}(\varphi)\left(C_{j}, \ldots, C_{j}, C_{j+1}, \ldots, C_{j+1}\right)=(-1)^{k} \varphi\left(\tilde{C}_{j+1}\right),
$$

if the vector field $C_{j}$ appears $k$ times in the left-hand side of this equation. Furthermore, if $p$ is odd, for $1 \leq j<k \leq n$, we also know that

$$
\tilde{\sigma}_{p}^{\prime}(\varphi)\left(A_{j k}, \ldots, A_{j k}\right)=\tilde{\sigma}_{p}^{\prime}(\varphi)\left(B_{j k}, \ldots, B_{j k}\right)=\varphi\left(\tilde{C}_{j}+\tilde{C}_{k}\right) .
$$

The following lemma is a direct consequence of the definitions of the forms $\sigma_{2}^{\prime}$ and $\sigma_{3}^{\prime}$.

Lemma 2.1. Let $A, B, C$ be elements of $\mathfrak{g}_{0}$ and $c \in \mathbb{R}$ satisfying

$$
A \cdot B+B \cdot A=i c C .
$$

If $\hat{A}, \hat{B}$ and $\hat{C}$ are the left-invariant vector fields on $G$ corresponding to $A, B$ and $C$, respectively, then we have

$$
\tilde{\sigma}^{\prime}(\varphi)(\hat{A}, \hat{B})=\frac{c}{2} \varphi(\hat{C}),
$$

for all $\varphi \in C^{\infty}\left(T^{*}\right)$.

Let $\mathcal{U}$ be the set of vectors $\left\{A_{j k}, B_{j k}\right\}$ of $\mathfrak{g}_{0}$, with $1 \leq j<k \leq n$. If $A, B$ are elements of $\mathcal{U}$, with $A \neq B$, and $1 \leq l \leq n-1$ is a given integer, then we easily verify that the relation (2.7) holds, where $C$ is equal to an element of $\mathcal{U}$ and $c=0$ or \pm 1 , and that

$$
A \cdot C_{l}+C_{l} \cdot A=i c A
$$

as elements of $M_{n}$, where $c=0$ or \pm 1 . By this remark, Lemma 2.1 and the relations $(2.3)-(2.6)$, we see that

$$
2 \tilde{\sigma}^{\prime}\left(\omega_{1}+\omega_{n-1}\right)=\omega_{1}^{2}-\omega_{n-1}^{2}+\sum_{j=1}^{n}\left(\omega_{1 j}^{2}+\varpi_{1 j}^{2}-\omega_{j n}^{2}-\varpi_{j n}^{2}\right) .
$$

For $1 \leq j \leq n-1$, since we have

$$
C_{j} \cdot A_{j, j+1}+A_{j, j+1} \cdot C_{j}=0
$$

as elements of $M_{n}$, by Lemma 2.1 we see that

$$
\tilde{\sigma}^{\prime}(\varphi)\left(C_{j}, A_{j, j+1}\right)=0,
$$


for all $\varphi \in C^{\infty}\left(T^{*}\right)$. From Lemma 2.1 and the previous remarks, we also deduce that

$$
\begin{aligned}
2 \tilde{\sigma}^{\prime}\left(\omega_{j k}\right)=\left(\omega_{j}-\omega_{j-1}+\omega_{k}\right. & \left.-\omega_{k-1}\right) \cdot \omega_{j k} \\
& +\sum_{l=1}^{n}\left(\omega_{j l} \cdot \varpi_{k l}-\omega_{k l} \cdot \varpi_{j l}\right), \\
2 \tilde{\sigma}^{\prime}\left(\varpi_{j k}\right)=\left(\omega_{j}-\omega_{j-1}+\omega_{k}-\omega_{k-1}\right) \cdot \varpi_{j k} & \\
& +\sum_{l=1}^{n}\left(\omega_{j l} \cdot \omega_{k l}+\varpi_{j l} \cdot \varpi_{k l}\right),
\end{aligned}
$$

for all $1 \leq j<k \leq n$.

When $n=3$, we know that the homogeneous polynomials $Q_{2}$ and $Q_{3}$ are algebraically independent generators of the algebra of $G$-invariant polynomials on $\mathfrak{g}_{0}$; hence the symmetric 4 -form $\sigma_{4}^{\prime}$ is a multiple of $\sigma_{2}^{\prime} \cdot \sigma_{2}^{\prime}=g^{\prime} \cdot g^{\prime}$. In fact, we verify that

$$
12 \sigma_{4}^{\prime}=g^{\prime} \cdot g^{\prime}
$$

From the preceding formula and (1.4), we deduce the following:

Lemma 2.2. When $n=3$, we have

$$
D^{2}\left(f g^{\prime}\right)=2 \tilde{\sigma}_{4}^{\prime}(d f)
$$

for all $f \in C_{\mathbb{R}}^{\infty}(X)$.

We now consider the mapping

$$
\iota^{\prime}: \mathbb{R}^{n-1} \rightarrow G
$$

of $[5, \S 3]$, which sends $\theta=\left(\theta_{1}, \ldots, \theta_{n-1}\right) \in \mathbb{R}^{n-1}$ into the diagonal matrix

$$
\iota^{\prime}(\theta)=\operatorname{diag}\left(e^{i x_{1}}, \ldots, e^{i x_{n}}\right)
$$

of $G$, where

$$
x_{1}=\theta_{1}, \quad x_{j}=\theta_{j}-\theta_{j-1}, \quad x_{n}=-\theta_{n-1},
$$

for $2 \leq j \leq n-1$. If $\left\{e_{1}^{\prime}, \ldots, e_{n-1}^{\prime}\right\}$ is the standard basis of $\mathbb{R}^{n-1}$ and $\Lambda^{\prime}$ is the lattice of $\mathbb{R}^{n-1}$ generated by the basis $\left\{2 \pi e_{j}^{\prime}\right\}_{1 \leq j \leq n-1}$ of $\mathbb{R}^{n-1}$, the mapping $\iota^{\prime}$ induces by passage to the quotient an imbedding

$$
\iota^{\prime}: \mathbb{R}^{n-1} / \Lambda^{\prime} \rightarrow G .
$$


The image of the mappings $\iota^{\prime}$ is the maximal torus $H$ of the group $G$ which consists of all diagonal matrices of $G$ and is therefore a maximal flat totally geodesic torus of $G$ viewed as a symmetric space. Clearly we have $\iota^{\prime}(0)=e_{0}$.

We consider the standard coordinate system $\left(\theta_{1}, \ldots, \theta_{n-1}\right)$ on $\mathbb{R}^{n-1}$ and endow this space with the Riemannian metric

$$
\tilde{g}=\sum_{j=1}^{n-1} d \theta_{j} \cdot d \theta_{j}-\sum_{j=1}^{n-2} d \theta_{j} \cdot d \theta_{j+1} .
$$

For $1 \leq j \leq n-1$, we consider the vector field $\xi_{j}=\partial / \partial \theta_{j}$ on $\mathbb{R}^{n-1}$. In [5, $\left.\S 3\right]$, we saw that the vector field $\zeta_{j}^{\prime}$ on $H$, determined by

$$
\iota_{*}^{\prime}\left(\xi_{j}(\theta)\right)=\zeta_{j}^{\prime}\left(\iota^{\prime}(\theta)\right)
$$

for $\theta \in \mathbb{R}^{n-1}$, is equal to the restriction of the vector field $C_{j}$ to $H$ and that the mapping $\iota^{\prime}: \mathbb{R}^{n-1} \rightarrow H$ is an isometric imbedding; moreover, we proved the following result given by Lemma 3.1 of [5]:

Lemma 2.3. Let $n, p \geq 3$ be given integers and let $X$ be the symmetric space $S U(n)$. A 1-form $\varphi$ on $X$ satisfies the Guillemin condition if and only if the symmetric $(p-1)$-form $\tilde{\sigma}_{p}^{\prime}(\varphi)$ on $X$ satisfies the Guillemin condition.

\section{Functions on the special unitary group}

Let $k \geq 1$ be a given integer. For $1 \leq j, l \leq k$, we denote by $z_{j l}$ the function on the space of matrices $M_{k}$ which sends a matrix of $M_{k}$ into its $(j, l)$-th entry. We also consider the complex-valued function $\Delta_{j l}^{(k)}$ on $M_{k}$ defined as follows. If $k=1$, the function $\Delta_{11}^{(1)}$ is identically equal to 1 ; if $k \geq 2$, the value of the function $\Delta_{j l}^{(k)}$ at a matrix $A \in M_{k}$ is the cofactor of the entry $z_{j l}(A)$ in $A$, which is equal to $(-1)^{j+l}$ times the determinant of the $(k-1) \times(k-1)$ matrix obtained from $A$ by deleting its $j$-th row and its $l$-th column. We note that, if $A \in M_{k}$ is a

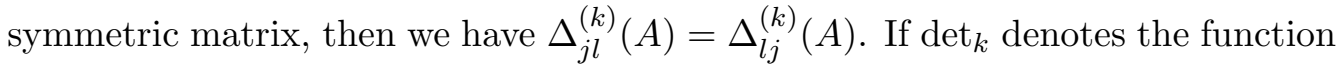
on $M_{k}$ which sends a matrix of $M_{k}$ into its determinant, we recall that

$$
\sum_{r=1}^{k} z_{j r} \Delta_{l r}^{(k)}=\sum_{r=1}^{k} z_{r j} \Delta_{r l}^{(k)}=\delta_{j l} \operatorname{det}_{k}
$$

for all $1 \leq j, l \leq k$. Thus we obtain the relation

$$
\frac{\partial}{\partial z_{j l}} \operatorname{det}_{k}=\Delta_{j l}^{(k)}
$$


for all $1 \leq j, l \leq k$.

We consider the group $G=S U(n)$, with $n \geq 3$, as a real submanifold of the complex manifold $M_{n}$. The left action and the right action of the group $G$ on the manifold $M_{n}$ induce morphism $\Phi$ and $\Phi^{\prime}$, respectively, from $\mathfrak{g}_{0}$ to the Lie algebra of vector fields on $M_{n}$ which are tangent to the submanifold $G$ of $M_{n}$. The mappings $\Phi$ and $\Phi^{\prime}$ extend to $\mathbb{C}$-linear morphisms from the complexification $\mathfrak{g}$ of $\mathfrak{g}_{0}$ to the space of all complex vector fields on $M_{n}$. For $\xi \in \mathfrak{g}_{0}$, the restriction of $-\Phi(\xi)$ (resp. of $\Phi^{\prime}(\xi)$ ) to $G$ is the right-invariant (resp. left-invariant) vector field on $G$ whose value at $e_{0}$ is the vector $\xi$ of $\mathfrak{g}_{0}=T_{e_{0}}(G)$; thus we have $\Phi(\xi)\left(e_{0}\right)=-\Phi^{\prime}(\xi)\left(e_{0}\right)$.

The functions $\left\{z_{j k}\right\}$ on $M_{n}$ defined above, with $1 \leq j, k \leq n$, form a holomorphic coordinate system for $M_{n}$. For $1 \leq j, k \leq n$, we consider the complex vector fields

$$
\xi_{j k}=\sum_{l=1}^{n} z_{j l} \frac{\partial}{\partial z_{k l}}, \quad \xi_{j k}^{\prime}=\sum_{l=1}^{n} z_{l j} \frac{\partial}{\partial z_{l k}}
$$

on $M_{n}$. For $1 \leq j, k \leq n$, with $j \neq k$, and $1 \leq l \leq n-1$, we verify that

$$
\begin{gathered}
\Phi\left(A_{j k}\right)=\bar{\xi}_{j k}-\bar{\xi}_{k j}+\xi_{j k}-\xi_{k j}, \quad \Phi\left(B_{j k}\right)=i\left(\bar{\xi}_{j k}+\bar{\xi}_{k j}-\xi_{j k}-\xi_{k j}\right), \\
\Phi\left(C_{l}\right)=i\left(\bar{\xi}_{l l}-\bar{\xi}_{l+1, l+1}+\xi_{l+1, l+1}-\xi_{l l}\right), \\
\Phi^{\prime}\left(A_{j k}\right)=\xi_{j k}^{\prime}-\xi_{k j}^{\prime}+\bar{\xi}_{j k}^{\prime}-\bar{\xi}_{k j}^{\prime}, \quad \Phi^{\prime}\left(B_{j k}\right)=i\left(\xi_{j k}^{\prime}+\xi_{k j}^{\prime}-\bar{\xi}_{j k}^{\prime}-\bar{\xi}_{k j}^{\prime}\right), \\
\Phi^{\prime}\left(C_{l}\right)=i\left(\xi_{l l}^{\prime}-\xi_{l+1, l+1}^{\prime}-\bar{\xi}_{l l}^{\prime}+\bar{\xi}_{l+1, l+1}^{\prime}\right) .
\end{gathered}
$$

If $1 \leq j, k \leq n$, with $j \neq k$, since $E_{j k}$ is equal to $\frac{1}{2}\left(A_{j k}-i B_{j k}\right)$, the complex vector fields $\eta_{j k}=\Phi\left(E_{j k}\right)$ and $\eta_{j k}^{\prime}=\Phi^{\prime}\left(E_{j k}\right)$ on $M_{n}$ are given by

$$
\eta_{j k}=\bar{\xi}_{j k}-\xi_{k j}, \quad \eta_{j k}^{\prime}=\xi_{j k}^{\prime}-\bar{\xi}_{k j}^{\prime},
$$

and so we have

$$
\bar{\eta}_{j k}=-\eta_{k j}
$$

Let $1 \leq k \leq n-1$ be a given integer. We denote by $J_{k}$ the set of all sequences $\left\{j_{1}, \ldots, j_{k}\right\}$ of integers satisfying $1 \leq j_{1}<\ldots<j_{k} \leq n$. If $\alpha=\left\{j_{1}, \ldots, j_{k}\right\}$ is an element of $J_{k}$, we consider the $M_{k}$-valued functions

$$
F_{j_{1} \ldots j_{k}}=\left(z_{l j_{r}}\right)_{1 \leq l, r \leq k}, \quad F_{j_{1} \ldots j_{k}}^{\prime}=\left(z_{j_{r} l}\right)_{1 \leq l, r \leq k}
$$


on $M_{n}$ and the complex-valued functions

$$
\phi_{\alpha}=\phi_{j_{1} \ldots j_{k}}=\operatorname{det} F_{j_{1} \ldots j_{k}}, \quad \phi_{\alpha}^{\prime}=\phi_{j_{1} \ldots j_{k}}=\operatorname{det} F_{j_{1} \ldots j_{k}}^{\prime}
$$

on $M_{n}$. For $1 \leq j, l \leq n$, we consider the function $\Delta_{j l}=\Delta_{j l}^{(n)}$ on $M_{n}$; clearly, we have

$$
\phi_{j}=z_{1 j}, \quad \phi_{j}^{\prime}=z_{j 1}, \quad \phi_{j_{1} \ldots j_{n-1}}=(-1)^{n+l} \Delta_{n l}, \quad \phi_{j_{1} \ldots j_{n-1}}^{\prime}=(-1)^{n+l} \Delta_{l n},
$$

where $j_{r}=r$, for $1 \leq r \leq l-1$, and $j_{r}=r+1$, for $l \leq r \leq n-1$. For $1 \leq j, k \leq n$, by (3.1) we easily verify that the equality

$$
\Delta_{j k}=\bar{z}_{j k},
$$

holds on the group $G$.

For $1 \leq k \leq n-1$, we write

$$
\phi_{(k)}=\phi_{\alpha}, \quad \phi_{(k)}^{\prime}=\phi_{\alpha}^{\prime},
$$

where $\alpha=\{n-k+1, \ldots, n\}$; in particular, we have

$$
\phi_{(1)}=z_{1 n}, \quad \phi_{(1)}^{\prime}=z_{n 1} .
$$

It is easily seen that the exterior power of a special unitary matrix is again special unitary; from the considerations of $[1, \S 6$, no. 3] and the formulas (9) and (11) of $[1, \S 6$, no. 4$]$, we then obtain the equality

$$
\phi_{(n-k)}^{\prime}=(-1)^{k(n+k)} \bar{\phi}_{(k)}
$$

on the group $G$. We note that the preceding relation, with $n=1$ or $n-1$, is given by (3.4).

Lemma 3.1. Let $1 \leq k \leq n-1$ and $1 \leq j, l \leq n$ be given integers. If $1 \leq j_{1}<\cdots<j_{k} \leq n$ are given integers, the equalities

$$
\xi_{j l} \phi_{j_{1} \ldots j_{k}}=\xi_{j l}^{\prime} \phi_{j_{1} \ldots j_{k}}=0
$$

hold on $M_{n}$ when $l>k$; the equalities

$$
\xi_{j l} \phi_{j_{1} \ldots j_{k}}=\delta_{j l} \phi_{j_{1} \ldots j_{k}}, \quad \xi_{j l}^{\prime} \phi_{j_{1} \ldots j_{k}}^{\prime}=\delta_{j l} \phi_{j_{1} \ldots j_{k}}^{\prime}
$$

hold on $M_{n}$ when $1 \leq j, l \leq k$. 
Proof. The first set of equalities are immediate. If $1 \leq j, l \leq k$, according to (3.1) and (3.2) we see that

$$
\xi_{j l} \phi_{j_{1} \ldots j_{k}}=\sum_{r=1}^{k} z_{j j_{r}} \Delta_{l r}^{(k)}\left(F_{j_{1} \ldots j_{k}}\right)=\sum_{r=1}^{k}\left(\Delta_{l r}^{(k)} z_{j r}\right)\left(F_{j_{1} \ldots j_{k}}\right)=\delta_{j l} \phi_{j_{1} \ldots j_{k}} ;
$$

a similar computation gives us the last equality of the lemma.

Let $1 \leq k \leq n-1$ and $1 \leq j<l \leq n$ be given integers. The preceding lemma implies that

$$
\eta_{l j} \phi_{j_{1} \ldots j_{k}}=-\xi_{j l} \phi_{j_{1} \ldots j_{k}}=\eta_{j l}^{\prime} \phi_{j_{1} \ldots j_{k}}^{\prime}=\xi_{j l}^{\prime} \phi_{j_{1} \ldots j_{k}}^{\prime}=0,
$$

for all integers $1 \leq j_{1}<\cdots<j_{k} \leq n$, and hence that

$$
\Phi\left(C_{j}\right) \phi_{j_{1} \ldots j_{k}}=-i \delta_{j k} \phi_{j_{1} \ldots j_{k}}, \quad \Phi^{\prime}\left(C_{j}\right) \phi_{j_{1} \ldots j_{k}}^{\prime}=i \delta_{j k} \phi_{j_{1} \ldots j_{k}}^{\prime}
$$

whenever $1 \leq j \leq n-1$.

Let $1 \leq k \leq n-1$ and $1 \leq j_{1}<\cdots<j_{k} \leq n$ be given integers; let $\left\{r_{1}, \ldots, r_{n-k}\right\}$ be the integers which do not belong to the set $\left\{j_{1}, \ldots, j_{k}\right\}$ and which satisfy $1 \leq r_{1}<\cdots<r_{n-k} \leq n$. Let $P_{j_{1} \ldots j_{k}}$ be the unique matrix of $M_{n}$ of determinant 1 whose $j_{l}$-th column is equal to the $l$-th column of the identity matrix $I_{n}$, for $1 \leq l \leq k$, and whose $r_{q}$-th column is equal to the $(k+q)$-th column of the identity matrix $I_{n}$, for $1 \leq q \leq n-k-1$, and whose $r_{n-k}$-th column is equal to the $n$-th column of the matrix $\varepsilon I_{n}$, where $\varepsilon= \pm 1$; clearly, $P_{j_{1} \ldots j_{k}}$ is an element of $S U(n)$. Then we see that

$$
\phi_{j_{1} \ldots j_{k}}\left(P_{l_{1} \ldots l_{k}}\right)=\delta_{j_{1} l_{1}} \cdots \delta_{j_{k} l_{k}}
$$

for all integers $1 \leq l_{1}<\cdots<l_{k} \leq n$.

Here and in subsequent sections, we shall consider the restrictions to $G$ of the functions $\phi_{j_{1} \ldots j_{k}}, \phi_{\alpha}, \phi_{j_{1} \ldots j_{k}}^{\prime}$ and $\phi_{\alpha}^{\prime}$ on $M_{n}$, where $\alpha \in J_{k}$, and denote them also by $\phi_{j_{1} \ldots j_{k}}, \phi_{\alpha}, \phi_{j_{1} \ldots j_{k}}^{\prime}$ and $\phi_{\alpha}^{\prime}$, respectively. From (3.9) it follows that the $\left(\begin{array}{l}n \\ k\end{array}\right)$ functions $\left\{\phi_{\alpha}\right\}$ on $G$, with $\alpha \in J_{k}$, are linearly independent; a similar argument shows that the $\left(\begin{array}{l}n \\ k\end{array}\right)$ functions $\left\{\phi_{\alpha}^{\prime}\right\}$ on $G$, with $\alpha \in J_{k}$, are also linearly independent.

For $1 \leq j, k, r, s \leq n$, with $r<s$, and $1 \leq l \leq n-1$, we have

$$
\begin{aligned}
\Phi^{\prime}\left(C_{l}\right) z_{j k} & =i z_{j k}\left(\delta_{k l}-\delta_{k-1, l}\right), \\
\Phi^{\prime}\left(A_{r s}\right) z_{j k} & =\left(\xi_{r s}^{\prime}-\xi_{s r}^{\prime}\right) z_{j k}=z_{j r} \delta_{k s}-z_{j s} \delta_{k r}, \\
\Phi^{\prime}\left(B_{r s}\right) z_{j k} & =i\left(\xi_{r s}^{\prime}+\xi_{s r}^{\prime}\right) z_{j k}=i\left(z_{j r} \delta_{k s}+z_{j s} \delta_{k r}\right) .
\end{aligned}
$$


It follows that the relation

$$
d z_{j k}=i z_{j k}\left(\omega_{k}-\omega_{k-1}\right)+\sum_{l=1}^{n} z_{j l}\left(\omega_{l k}+i \varpi_{l k}\right)
$$

holds on $G$, for $1 \leq j, k \leq n$.

By means of the formulas (2.2), (3.11), (2.8) and (2.10), we verify directly the following result:

Lemma 3.2. We have

$$
i D^{1}\left(z_{n 1} d \bar{z}_{1 n}-\bar{z}_{1 n} d z_{n 1}\right)=\tilde{\sigma}^{\prime} d\left(z_{n 1} \bar{z}_{1 n}\right) .
$$

\section{Highest Weight VeCtors AND FUnCtions ON $S U(n)$}

We consider the maximal torus $H$ of the simple group $G$ introduced in $\S 2$ and its Lie algebra $\mathfrak{h}_{0}$, and also the complexification $\mathfrak{g}=\mathfrak{s} \mathfrak{l}(n, \mathbb{C})$ of the Lie algebra $\mathfrak{g}_{0}$ of $G$. The complexification $\mathfrak{h}$ of $\mathfrak{h}_{0}$ is equal to the Cartan subalgebra of the simple Lie algebra $\mathfrak{g}$ consisting of all diagonal matrices of $\mathfrak{g}$, and the matrices $\left\{C_{1}, \ldots, C_{n-1}\right\}$ form a basis of $\mathfrak{h}_{0}$. For $1 \leq j \leq n$, the linear form $\lambda_{j}: \mathfrak{h} \rightarrow \mathbb{C}$, sending the diagonal matrix with $a_{1}, \ldots, a_{n} \in \mathbb{C}$ as its diagonal entries into $a_{j}$, is purely imaginary on $\mathfrak{h}_{0}$. We write $\alpha_{j}=\lambda_{j}-\lambda_{j+1}$, for $1 \leq j \leq n-1$. Then

$$
\left\{\lambda_{j}-\lambda_{k} \mid 1 \leq j, k \leq n \text { and } j \neq k\right\}
$$

is the system of roots of $\mathfrak{g}$ with respect to $\mathfrak{h}$. As in $[5, \S 5]$, we take $\left\{\alpha_{1}, \ldots, \alpha_{n-1}\right\}$ as a system of simple roots of $\mathfrak{g}$; the corresponding system of positive roots is

$$
\Delta^{+}=\left\{\lambda_{j}-\lambda_{k} \mid 1 \leq j<k \leq n\right\} .
$$

If $\alpha$ is the root $\lambda_{j}-\lambda_{k}$, with $1 \leq j, k \leq n$ and $j \neq k$, the root subspace $\mathfrak{g}_{\alpha}$ corresponding to $\alpha$ is generated by $E_{j k}$ (over $\mathbb{C}$ ). We have the decomposition

$$
\mathfrak{g}=\mathfrak{n}^{-} \oplus \mathfrak{h} \oplus \mathfrak{n}^{+},
$$

where

$$
\mathfrak{n}^{+}=\bigoplus_{\alpha \in \Delta^{+}} \mathfrak{g}_{\alpha}, \quad \mathfrak{n}^{-}=\bigoplus_{\alpha \in \Delta^{+}} \mathfrak{g}_{-\alpha}
$$

The corresponding fundamental weights are

$$
\varpi_{j}=\lambda_{1}+\cdots+\lambda_{j}
$$


with $1 \leq j \leq n-1$; in fact, $\varpi_{j}$ is the highest weight of the irreducible $G$-module $\bigwedge^{j} \mathbb{C}^{n}$, and we have

$$
\varpi_{k}\left(C_{j}\right)=i \delta_{j k},
$$

for $1 \leq j, k \leq n-1$. The unique element $w_{0}$ of the Weyl group of $\mathfrak{g}$ determined

$$
w_{0}\left(\Delta^{+}\right)=-\Delta^{+}
$$

is the involutive automorphism satisfying

$$
w_{0}\left(\varpi_{j}\right)=-\varpi_{n-j},
$$

for $1 \leq j \leq n-1$. A dominant integral form $\lambda$ for $G$ may be written in a unique way

$$
\lambda=\gamma_{r_{1}, \ldots, r_{n-1}}=r_{1} \varpi_{1}+\cdots+r_{n-1} \varpi_{n-1},
$$

where $r_{1}, \ldots, r_{n-1}$ are non-negative integers. Thus the highest weight of an irreducible (complex) $G$-module has a unique expression of this form and we may identify the dual $\Gamma$ of $G$ with the set of all linear forms on $\mathfrak{h}$ which can be written in the form (4.4).

If $\gamma=\gamma_{r_{1}, r_{2}, \ldots, r_{n-1}}$ is an element of $\Gamma$, where $r_{1}, r_{2}, \ldots, r_{n-1}$ are non-negative integers, let $E_{\gamma}$ be an irreducible $G$-module corresponding to $\gamma$. By (4.3), the unique element $\bar{\gamma}$ of $\Gamma$ determined by

$$
w_{0}(\gamma)=-\bar{\gamma}
$$

is equal to $\gamma_{r_{n-1}, \ldots, r_{2}, r_{1}}$; in particular, if $\gamma$ is the element $\varpi_{k}$ of $\Gamma$, we have $\bar{\gamma}=\varpi_{n-k}$. A linear form $\lambda$ on $\mathfrak{h}$ is a weight of the $G$-module $E_{\gamma}$ if and only if $-\lambda$ is a weight of the contragredient $G$-module $E_{\gamma}^{*}$ of $E_{\gamma}$. Therefore the $G$-module $E_{\bar{\gamma}}$ is isomorphic to $E_{\gamma}^{*}$.

We consider the group $\tilde{G}=G \times G$ and its Lie algebra $\tilde{\mathfrak{g}}_{0}$. We identify the complexification $\tilde{\mathfrak{g}}=\mathfrak{g} \oplus \mathfrak{g}$ of $\tilde{\mathfrak{g}}_{0}$ with a subalgebra of $\mathfrak{s l}(2 n, \mathbb{C})$ and consider the Cartan subalgebra $\tilde{\mathfrak{h}}$ of $\tilde{\mathfrak{g}}$ consisting of all its diagonal matrices. For $1 \leq j \leq 2 n$, we consider the linear form $\lambda_{j}: \tilde{\mathfrak{h}} \rightarrow \mathbb{C}$ which sends the diagonal matrix with $a_{1}, \ldots, a_{2 n} \in \mathbb{C}$ as its diagonal entries into $a_{j}$. We write $\alpha_{j}=\lambda_{j}-\lambda_{j+1}$ and $\beta_{j}=\lambda_{j+n}-\lambda_{j+n+1}$, for $1 \leq j \leq n-1$. We take $\left\{\alpha_{1}, \ldots, \alpha_{n-1}, \beta_{1}, \ldots, \beta_{n-1}\right\}$ as a system of simple roots of $\tilde{\mathfrak{g}}$ with respect to $\tilde{\mathfrak{h}}$. We may then identify the dual of the group $\tilde{G}=G \times G$ with the product $\Gamma \times \Gamma$; in fact, if $\gamma_{1}, \gamma_{2}$ are elements of $\Gamma$, then $E_{\gamma_{1}} \otimes E_{\gamma_{2}}$ is an irreducible $\tilde{G}$-module corresponding to $\left(\gamma_{1}, \gamma_{2}\right)$. 
We now consider the $\tilde{G}$-module structures on $C^{\infty}(G)$ and $C^{\infty}\left(S^{p} T_{\mathbb{C}}^{*}\right)$ induced by the action of $\tilde{G}$ on $X$ and the corresponding representations of $\tilde{G}$ or the Lie algebra $\tilde{\mathfrak{g}}$ on these modules. According to the Peter-Weyl theorem, the isotypic component $C_{\left(\gamma^{\prime}, \gamma\right)}^{\infty}(G)$ of the $\tilde{G}$-module $C^{\infty}(G)$ corresponding to the element $\left(\gamma^{\prime}, \gamma\right)$ of $\Gamma \times \Gamma$ vanishes unless $\gamma^{\prime}=\bar{\gamma}$, and is equal to the image of the morphism of $\tilde{G}$-modules

$$
A_{\gamma}: E_{\gamma}^{*} \otimes E_{\gamma} \rightarrow C^{\infty}(G)
$$

defined by

$$
A_{\gamma}(\alpha \otimes v)(g)=\alpha(g v),
$$

for $\alpha \in E_{\gamma}^{*}, v \in E_{\gamma}$ and $g \in G$, when $\gamma^{\prime}=\bar{\gamma}$. Thus for $\gamma \in \Gamma$, the isotypic component $C_{(\bar{\gamma}, \gamma)}^{\infty}(G)$ is an irreducible $\tilde{G}$-submodule of $C^{\infty}(G)$.

The spaces $C^{\infty}(G)$ and $C^{\infty}\left(S^{p} T_{\mathbb{C}}^{*}\right)$ inherit structures of $G$-modules arising from the left (resp. right) action of $G$ on $X$. The corresponding representation $\pi$ (resp. $\pi^{\prime}$ ) of $G$ on $C^{\infty}(G)$ is the left (resp. right) regular representation; we shall also consider the corresponding representation $\left(\pi, C^{\infty}\left(S^{p} T_{\mathbb{C}}^{*}\right)\right)$ (resp. $\left(\pi^{\prime}, C^{\infty}\left(S^{p} T_{\mathbb{C}}^{*}\right)\right)$ ) of $G$ or of the Lie algebra $\mathfrak{g}$ on $C^{\infty}\left(S^{p} T_{\mathbb{C}}^{*}\right)$. If $\xi_{1}, \xi_{2}$ are elements of $\mathfrak{g}_{0}$ and $u$ is an element of $C^{\infty}(G)$ or $C^{\infty}\left(S^{p} T_{\mathbb{C}}^{*}\right)$, we have

$$
\pi\left(\xi_{1}\right) \pi^{\prime}\left(\xi_{2}\right) u=\pi^{\prime}\left(\xi_{2}\right) \pi\left(\xi_{1}\right) u
$$

Moreover, if $\xi \in \mathfrak{g}_{0}$ and $f \in C^{\infty}(G)$, we have

$$
\pi(\xi) f=\Phi(\xi) f, \quad \pi^{\prime}(\xi) f=\Phi^{\prime}(\xi) f
$$

and so the relations

$$
\pi(\xi) \cdot f=\Phi(\xi) \cdot f=-\Phi^{\prime}(\xi) \cdot f=-\pi^{\prime}(\xi) \cdot f=-\xi \cdot f
$$

hold at the point $e_{0}$; in the expression $\xi \cdot f$ of these equalities, $\xi$ is considered as a left-invariant vector field on $G$.

If $\gamma$ is an element of $\Gamma$, from the above remarks concerning the Peter-Weyl theorem it follows that the isotypic component $C_{\gamma}^{\infty}(G)$ of the $G$-module $\left(\pi, C^{\infty}(G)\right)$ corresponding to $\gamma$ is equal to the irreducible $\tilde{G}$-module $C_{(\gamma, \bar{\gamma})}^{\infty}(G)$; it is therefore isomorphic to $k$ copies of $E_{\gamma}$, where the integer $k$ is the dimension of $E_{\gamma}$ (over $\mathbb{C}$ ). Thus the dimension of the weight subspace $\mathcal{C}_{\gamma}$ of the $G$-submodule $C_{\gamma}^{\infty}(G)$ corresponding to its highest weight $\gamma$ is equal to the dimension of $E_{\gamma}$. Clearly, the space $C_{\gamma}^{\infty}(G)=C_{(\gamma, \bar{\gamma})}^{\infty}(G)$ and the weight subspace $\mathcal{C}_{\gamma}$ are $G$-submodules 
of $\left(\pi^{\prime}, C^{\infty}(G)\right)$. The subspace $\mathcal{C}_{\bar{\gamma}}^{\prime}$ of $C^{\infty}(G)$ generated by the highest weight vectors of weight $\bar{\gamma}$ of the representation $\left(\pi^{\prime}, C^{\infty}(G)\right)$ is a $G$-submodule of $C_{\gamma}^{\infty}(G)$ whose dimension is also equal to the dimension of $E_{\gamma}$. We also know that $\mathcal{C}_{\gamma}$ is an irreducible $G$-module whose highest weight is $\bar{\gamma}$ and that $\mathcal{C}_{\bar{\gamma}}^{\prime}$ is an irreducible $G$-submodule of $C_{\gamma}^{\infty}(G)$. Moreover, the intersection

$$
\tilde{\mathcal{C}}_{\gamma}=\mathcal{C}_{\gamma} \cap \mathcal{C}_{\bar{\gamma}}^{\prime}
$$

is the weight subspace of the irreducible $\tilde{G}$-submodule $C_{(\gamma, \bar{\gamma})}^{\infty}(G)$ corresponding to its highest weight $(\gamma, \bar{\gamma})$, and is therefore one-dimensional.

As in $\S 1$, if $\left(\gamma, \gamma^{\prime}\right)$ is an element of $\Gamma \times \Gamma$, we denote by $C_{\left(\gamma, \gamma^{\prime}\right)}^{\infty}\left(S^{p} T_{\mathbb{C}}^{*}\right)$ the isotypic component of the $\tilde{G}$-module $C^{\infty}\left(S^{p} T_{\mathbb{C}}^{*}\right)$ corresponding to $\left(\gamma, \gamma^{\prime}\right)$. We shall denote by $C_{\gamma}^{\infty}\left(S^{p} T_{\mathbb{C}}^{*}\right)$ the isotypic component of the $G$-module $\left(\pi, C^{\infty}\left(S^{p} T_{\mathbb{C}}^{*}\right)\right)$ corresponding to $\gamma$ and by $\mathcal{C}_{\gamma}\left(S^{p} T_{\mathbb{C}}^{*}\right)$ the weight subspace of the $G$-module $C_{\gamma}^{\infty}\left(S^{p} T_{\mathbb{C}}^{*}\right)$ corresponding to its highest weight. The isotypic component $C_{\left(\gamma, \gamma^{\prime}\right)}^{\infty}\left(S^{p} T_{\mathbb{C}}^{*}\right)$ is a $\tilde{G}$-submodule of $C_{\gamma}^{\infty}\left(S^{p} T_{\mathbb{C}}^{*}\right)$ and the weight space $\mathcal{C}_{\left(\gamma, \gamma^{\prime}\right)}\left(S^{p} T_{\mathbb{C}}^{*}\right)$ of the $\tilde{G}$-module $C_{\left(\gamma, \gamma^{\prime}\right)}^{\infty}\left(S^{p} T_{\mathbb{C}}^{*}\right)$ corresponding to its highest weight $\left(\gamma, \gamma^{\prime}\right)$ is contained in the weight space of the $G$-submodule $\mathcal{C}_{\gamma}\left(S^{p} T_{\mathbb{C}}^{*}\right)$ of $\left(\pi^{\prime}, C_{\gamma}^{\infty}\left(S^{p} T_{\mathbb{C}}^{*}\right)\right)$ corresponding to the weight $\gamma^{\prime}$.

We now view the complexification $\mathcal{A}_{\mathbb{C}}$ of the space $\mathcal{A}$ of left-invariant 1-forms on $G$ as a $\tilde{G}$-submodule of $C^{\infty}\left(T_{\mathbb{C}}^{*}\right)$; more generally, if $p$ is an integer $\geq 1$, we view the $p$-th symmetric power $S^{p} \mathcal{A}$ of $\mathcal{A}$ and its complexification $S^{p} \mathcal{A}_{\mathbb{C}}$ as $\tilde{G}$-submodules of $C^{\infty}\left(S^{p} T_{\mathbb{C}}^{*}\right)$. Clearly, the space $\mathcal{A}_{\mathbb{C}}$ is a trivial $G$-submodule of $\left(\pi, C^{\infty}\left(T_{\mathbb{C}}^{*}\right)\right)$ and a $G$-submodule of $\left(\pi^{\prime}, C^{\infty}\left(T_{\mathbb{C}}^{*}\right)\right)$ isomorphic to the irreducible $G$-module $\mathfrak{g}$. Thus the space $S^{p} \mathcal{A}_{\mathbb{C}}$ is a trivial $G$-submodule of $\left(\pi, C^{\infty}\left(S^{p} T_{\mathbb{C}}^{*}\right)\right)$ and is also a $G$-submodule of $\left(\pi^{\prime}, C^{\infty}\left(S^{p} T_{\mathbb{C}}^{*}\right)\right)$.

Let $\gamma$ be an element of $\Gamma$ and $p$ be an integer $\geq 1$. Since the cotangent bundle $T^{*}$ of $G$ is trivial, if $V_{p}$ is the $G$-module equal to the vector space $S^{p} \mathfrak{g}$ endowed with the trivial action of $G$, the isotypic component $C_{\gamma}^{\infty}\left(S^{p} T_{\mathbb{C}}^{*}\right)$ is isomorphic to $C_{\gamma}^{\infty}(G) \otimes V_{p}$. Thus the weight space $\mathcal{C}_{\gamma}\left(S^{p} T_{\mathbb{C}}^{*}\right)$ is equal to $\mathcal{C}_{\gamma} \cdot S^{p} \mathcal{A}_{\mathbb{C}}$ and the weight space $\mathcal{C}_{(\gamma, \bar{\gamma})}\left(S^{p} T_{\mathbb{C}}^{*}\right)$ is contained in the weight space of the $G$-submodule $\mathcal{C}_{\gamma} \cdot S^{p} \mathcal{A}_{\mathbb{C}}$ of $\left(\pi^{\prime}, C_{\gamma}^{\infty}\left(S^{p} T_{\mathbb{C}}^{*}\right)\right)$ corresponding to the weight $\bar{\gamma}$. We shall denote by $\mathcal{W}_{\gamma}$ the weight subspace of the $G$-submodule $\mathcal{C}_{\gamma} \cdot \mathcal{A}_{\mathbb{C}}$ of $\left(\pi^{\prime}, C_{\gamma}^{\infty}\left(T_{\mathbb{C}}^{*}\right)\right)$ corresponding to the weight $\bar{\gamma}$. 
In the remainder of this section, if $\gamma$ is an element of $\Gamma$, we shall always consider the $G$-module structures on $C^{\infty}(G)$ and $C_{\gamma}^{\infty}(G)$ arising from the representation $\pi$ and the $G$-module structure on $\mathcal{C}_{\gamma}$ arising from the representation $\pi^{\prime}$.

If $\gamma \in \Gamma$, a linear form $\lambda$ on $\mathfrak{h}$ is a weight of the $G$-module $C_{\gamma}^{\infty}(G)$ if and only if $-\lambda$ is a weight of the complex conjugate $\overline{C_{\gamma}^{\infty}(G)}$ of the space $C_{\gamma}^{\infty}(G)$. Therefore we have the equalities

$$
C_{\bar{\gamma}}^{\infty}(G)=\overline{C_{\gamma}^{\infty}(G)}
$$

of $G$-modules. If $r \geq 1$ is a given integer, the element $\gamma=r\left(\varpi_{1}+\varpi_{n-1}\right)$ of $\Gamma$ satisfies $\bar{\gamma}=\gamma$; thus according to (4.6), the $G$-module $C_{\gamma}^{\infty}(G)$ is invariant under complex conjugation. In particular, if $\gamma$ is the element $\varpi_{1}+\varpi_{n-1}$ of $\Gamma$ the $G$-module $\mathcal{B}^{\prime}=C_{\gamma}^{\infty}(G)=C_{(\gamma, \gamma)}^{\infty}(G)$ is invariant under complex conjugation and is equal to the complexification of the $G$-submodule

$$
\mathcal{B}_{\mathbb{R}}^{\prime}=\left\{f \in \mathcal{B}^{\prime} \mid f=\bar{f}\right\}
$$

of $C_{\mathbb{R}}^{\infty}(G)$.

Let $1 \leq k \leq n-1$ and $1 \leq j_{1}<\cdots<j_{k} \leq n$ be given integers. From the relations (3.7), (3.8) and (4.2), we infer that the equalities

$$
\begin{aligned}
\Phi\left(C_{j}\right) \phi_{j_{1} \ldots j_{k}}=-\varpi_{k}\left(C_{j}\right) \phi_{j_{1} \ldots j_{k}}, & \Phi\left(E_{r j}\right) \phi_{j_{1} \ldots j_{k}}=0, \\
\Phi^{\prime}\left(C_{j}\right) \phi_{j_{1} \ldots j_{k}}^{\prime}=\varpi_{k}\left(C_{j}\right) \phi_{j_{1} \ldots j_{k}}^{\prime}, & \Phi^{\prime}\left(E_{j r}\right) \phi_{j_{1} \ldots j_{k}}^{\prime}=0
\end{aligned}
$$

hold on $M_{n}$ for all integers $1 \leq j<r \leq n$. From the relations (4.7) and (3.3), it follows that

$$
\begin{aligned}
\pi(\xi) \bar{\phi}_{j_{1} \ldots j_{k}} & =\varpi_{k}(\xi) \bar{\phi}_{j_{1} \ldots j_{k}}, & \pi(\eta) \bar{\phi}_{j_{1} \ldots j_{k}} & =0, \\
\pi^{\prime}(\xi) \phi_{j_{1} \ldots j_{k}}^{\prime} & =\varpi_{k}(\xi) \phi_{j_{1} \ldots j_{k}}^{\prime}, & \pi^{\prime}(\eta) \phi_{j_{1} \ldots j_{k}}^{\prime} & =0,
\end{aligned}
$$

for all $\xi \in \mathfrak{h}_{0}$ and $\eta \in \mathfrak{n}^{+}$. Thus the function $\bar{\phi}_{j_{1} \ldots j_{k}}$ (resp. $\phi_{j_{1} \ldots j_{k}}^{\prime}$ ) is an element of the $G$-module $\mathcal{C}_{\varpi_{k}}$ (resp. $\mathcal{C}_{\varpi_{k}}^{\prime}$ ); moreover according to (4.6), we know that $\phi_{j_{1} \ldots j_{k}}$ (resp. $\left.\bar{\phi}_{j_{1} \ldots j_{k}}^{\prime}\right)$ is an element of $C_{\varpi_{n-k}}^{\infty}(G)$.

We saw that the $\left(\begin{array}{l}n \\ k\end{array}\right)$ functions $\bar{\phi}_{\alpha}$ (resp. $\phi_{\alpha}^{\prime}$ ) on $G$, with $\alpha \in J_{k}$, are linearly independent; since the dimension of the space $\mathcal{C}_{\varpi_{k}}$ (resp. $\mathcal{C}_{\varpi_{k}}^{\prime}$ ) is equal to the dimension of the irreducible $G$-module $\bigwedge^{k} \mathbb{C}^{n}$, which corresponds to the weight $\varpi_{k}$, this set of functions is a basis of the space $\mathcal{C}_{\varpi_{k}}$ (resp. $\mathcal{C}_{\varpi_{k}}^{\prime}$ ).

Let $\gamma=\gamma_{r_{1}, r_{2}, \ldots, r_{n-1}}$ be an element of $\Gamma$, where $r_{1}, \ldots, r_{n-1}$ are non-negative integers. We consider the subspace $\mathcal{P}_{\gamma}$ of $C^{\infty}(G)$ generated (over $\mathbb{C}$ ) by the 
functions

$$
\prod_{k=1}^{n-1} \bar{\phi}_{\alpha_{k, 1}} \ldots \bar{\phi}_{\alpha_{k, r_{k}}}
$$

where $\alpha_{k, 1}, \ldots, \alpha_{k, r_{k}}$ are elements of $J_{k}$, for $1 \leq k \leq n-1$. From the previous observation, it follows that $\mathcal{P}_{\gamma}$ is a $G$-submodule of the irreducible $G$-module $\mathcal{C}_{\gamma}$. Since the module $\mathcal{P}_{\gamma}$ is non-zero, we therefore obtain the equality

$$
\mathcal{C}_{\gamma}=\mathcal{P}_{\gamma}
$$

The next proposition is a direct consequence of the above remarks and the relation (3.6). Here we shall only use this result for elements of $\Gamma$ of the form $s \varpi_{1}+r \varpi_{n-1}$, with $r, s \geq 0$; the corresponding statement is given by Lemma 4.2 and we remark that the proof of this lemma requires only the relations (3.4). However, for the sake of completeness, we present the most general assertion.

Proposition 4.1. For $1 \leq k \leq n-1$, the function $\bar{\phi}_{(k)}$ is a generator of the one-dimensional space

$$
\tilde{\mathcal{C}}_{\varpi_{k}}=\mathcal{C}_{\varpi_{k}} \cap \mathcal{C}_{\varpi_{n-k}}^{\prime}
$$

More generally, if $\gamma=\gamma_{r_{1}, r_{2}, \ldots, r_{n-1}}$ is an element of $\Gamma$, where $r_{1}, \ldots, r_{n-1}$ are non-negative integers, then the function

$$
\phi_{\gamma}=\prod_{k=1}^{n-1} \bar{\phi}_{(k)}^{r_{k}}
$$

on $G$ is a generator of the one-dimensional space $\tilde{\mathcal{C}}_{\gamma}$.

According to (3.6), the function $\phi_{\gamma}$ to $G$ is equal to the product

$$
\prod_{k=1}^{n-1} \phi_{(k)}^{\prime r_{n-k}}
$$

up to a sign.

According to Proposition 4.1 and (3.5), the one-dimensional spaces

$$
\tilde{\mathcal{C}}_{\varpi_{1}}=\mathcal{C}_{\varpi_{1}} \cap \mathcal{C}_{\varpi_{n-1}}^{\prime}, \quad \tilde{\mathcal{C}}_{\varpi_{n-1}}=\mathcal{C}_{\varpi_{n-1}} \cap \mathcal{C}_{\varpi_{1}}^{\prime}
$$

are generated by the functions $\bar{z}_{1 n}$ and $z_{n 1}$, respectively. By (3.4), the functions $\left\{\bar{z}_{1 j}\right\}_{1 \leq j \leq n}$ (resp. $\left\{z_{n j}\right\}_{1 \leq j \leq n}$ ) on $G$ form a basis of the space $\mathcal{C}_{\varpi_{1}}$ (resp. $\mathcal{C}_{\varpi_{n-1}}$ ); moreover, the functions $\left\{z_{j 1}\right\}_{1 \leq j \leq n}$ (resp. $\left\{\bar{z}_{j n}\right\}_{1 \leq j \leq n}$ ) on $G$ form a basis of the space $\mathcal{C}_{\varpi_{1}}^{\prime}$ (resp. $\mathcal{C}_{\varpi_{n-1}}^{\prime}$ ). Thus from the above discussion, we obtain the following: 
Lemma 4.2. Let $\gamma$ be the element $s \varpi_{1}+r \varpi_{n-1}$ of $\Gamma$, where $r, s \geq 0$ are integers. The irreducible $G$-module $\mathcal{C}_{\gamma}$ is generated (over $\mathbb{C}$ ) by the functions

$$
\left\{z_{n j_{1}} z_{n j_{2}} \cdots z_{n j_{r}} \bar{z}_{1 k_{1}} \bar{z}_{1 k_{2}} \cdots \bar{z}_{1 k_{s}}\right\}
$$

with $1 \leq j_{1}, j_{2}, \ldots, j_{r}, k_{1}, k_{2}, \ldots, k_{s} \leq n$ and the function $z_{n 1}^{r} \bar{z}_{1 n}^{s}$ is a highest weight vector of this $G$-module and of the $\tilde{G}$-module $C_{(\gamma, \bar{\gamma})}^{\infty}(G)$.

\section{Highest weight vectors and forms of Degree one on $S U(n)$}

We consider the group $X=G=S U(n)$, with $n \geq 3$. In this section, we shall consider the spaces $C^{\infty}(G)$ and $C^{\infty}\left(S^{p} T_{\mathbb{C}}^{*}\right)$ endowed with their $G$-module structures arising from the right action of $G$ on $X$. For $\gamma \in \Gamma$, we view the weight space $\mathcal{C}_{\gamma}$ as a $G$-submodule of $C^{\infty}(G)$ and the weight space $\mathcal{C}_{\gamma}\left(S^{p} T_{\mathbb{C}}^{*}\right)$ as a $G$-submodule of $C^{\infty}\left(S^{p} T_{\mathbb{C}}^{*}\right)$.

Proposition 5.1. Let $p \geq 2$ be a given integer and let $\gamma$ be a non-zero element of $\Gamma$. Then the following assertions are equivalent:

(i) The equality

$$
D^{p-1} C_{\gamma}^{\infty}\left(S^{p-1} T_{\mathbb{C}}^{*}\right) \cap \tilde{\sigma}_{p+1}^{\prime} d C_{\gamma}^{\infty}(X)=\{0\}
$$

holds.

(ii) The equality

$$
D^{p-1} C_{(\gamma, \bar{\gamma})}^{\infty}\left(S^{p-1} T_{\mathbb{C}}^{*}\right) \cap \tilde{\sigma}_{p+1}^{\prime} d C_{\gamma}^{\infty}(X)=\{0\}
$$

holds.

(iii) If $f$ is a highest weight vector of the $G$-module $\mathcal{C}_{\gamma}$, there does not exist a highest weight vector $u$ of the $\tilde{G}$-module $C_{(\gamma, \bar{\gamma})}^{\infty}\left(S^{p-1} T_{\mathbb{C}}^{*}\right)$ satisfying the relation

$$
D^{p-1} u=\tilde{\sigma}_{p+1}^{\prime}(d f) \text {. }
$$

Proof. We remark that the differential operators $D^{p-1}$ and $\tilde{\sigma}_{p+1}^{\prime} d$ are $\tilde{G}$-invariant. Since $C_{\gamma}^{\infty}(X)$ is equal to $C_{(\gamma, \bar{\gamma})}^{\infty}(X)$, we therefore see that assertions (i) and (ii) are equivalent. Moreover the left-hand side $M$ of the equality (5.2) is a $\tilde{G}$-submodule of $C_{(\gamma, \bar{\gamma})}^{\infty}\left(S^{p} T_{\mathbb{C}}^{*}\right)$. Suppose that the $\tilde{G}$-module $M$ does not vanish and let $w$ be a highest vector of this module; then we may write $w=D^{p-1} u=\tilde{\sigma}_{p+1}^{\prime} d f$, where $f$ and $u$ are highest vectors of the $G$-module $\mathcal{C}_{\gamma}$ and the $\tilde{G}$-module $\mathcal{C}_{(\gamma, \bar{\gamma})}\left(S^{p-1} T_{\mathbb{C}}^{*}\right)$, 
respectively. The equivalence of assertions (ii) and (iii) is a direct consequence of this last observation and the fact that $\tilde{\sigma}_{p+1}^{\prime}$ is a monomorphism.

For $1 \leq k \leq n$, according to (3.10) we see that the elements $z_{n k}$ and $\bar{z}_{1 k}$ of the $G$-module $C^{\infty}(G)$ are vectors of weight $\lambda_{k}$ and $-\lambda_{k}$, respectively. Thus if $r_{1}, \ldots, r_{n}, s_{1}, \ldots, s_{n} \geq 0$ are given integers, the element

$$
q_{r_{1}, \ldots, r_{n}, s_{1}, \ldots, s_{n}}=z_{n 1}^{r_{1}} \cdots z_{n n}^{r_{n}} \bar{z}_{11}^{s_{1}} \cdots \bar{z}_{1 n}^{s_{n}}
$$

of $C^{\infty}(G)$ is of weight $\left(r_{1}-s_{1}\right) \lambda_{1}+\cdots+\left(r_{n}-s_{n}\right) \lambda_{n}$.

For $1 \leq j<k \leq n$, we consider the elements

$$
\theta_{j k}=\omega_{j k}-i \varpi_{j k}, \quad \bar{\theta}_{j k}=\omega_{j k}+i \varpi_{j k}
$$

of $\mathcal{A}_{\mathbb{C}} ;$ then the set of 1 -forms

$$
\left\{\omega_{l}, \theta_{j k}, \bar{\theta}_{j k}\right\},
$$

with $1 \leq l \leq n-1$ and $1 \leq j<k \leq n$, is a basis of the $G$-module $\mathcal{A}_{\mathbb{C}}$. For $1 \leq l \leq n-1$, the element $\omega_{l}$ is a vector of $\mathcal{A}_{\mathbb{C}}$ of weight 0 ; on the other hand, for $1 \leq j<k \leq n$, the elements $\theta_{j k}$ and $\bar{\theta}_{j k}$ are vectors of $\mathcal{A}_{\mathbb{C}}$ of weight $\lambda_{j}-\lambda_{k}$ and $\lambda_{k}-\lambda_{j}$, respectively.

In this section, we shall use the results given by Lemma 4.2 with $r=s \geq 1$. We also remark that

$$
r\left(\varpi_{1}+\varpi_{n-1}\right)=r\left(\lambda_{1}-\lambda_{n}\right) .
$$

The following result is a direct consequence of Lemma 4.2, with $r=s$.

Lemma 5.2. Let $r \geq 1$ and $r_{1}, \ldots, r_{n}, s_{1}, \ldots, s_{n} \geq 0$ be given integers; suppose that $r_{1}+\cdots+r_{n}=s_{1}+\cdots+s_{n}=r$. If the element $q=q_{r_{1}, \ldots, r_{n}, s_{1}, \ldots, s_{n}}$ of $C^{\infty}(G)$ is of weight $r\left(\lambda_{1}-\lambda_{n}\right)$, then $r_{1}=s_{n}=r$ and the function $q$ is equal to $\left(z_{n 1} \bar{z}_{1 n}\right)^{r}$.

We now give an alternate proof of Lemma 5.2. Let $a_{1}, \ldots, a_{n}$ be elements of $\mathbb{C}$ satisfying $a_{1}+\cdots+a_{n}=0$, and consider the element $\xi=\sum_{j=1}^{n} a_{j} E_{j j}$ of the Cartan subalgebra $\mathfrak{h}$. Then we have

$$
\begin{aligned}
\pi^{\prime}(\xi) q & =\left(r_{1} a_{1}+\cdots+r_{n} a_{n}-s_{1} a_{1}-\cdots-s_{n} a_{n}\right) q \\
& =\left(\left(r_{1}-r_{n}-s_{1}+s_{n}\right) a_{1}+\cdots+\left(r_{n-1}-r_{n}-s_{n-1}+s_{n}\right) a_{n-1}\right) q .
\end{aligned}
$$

If the function $q$ is of weight $r\left(\lambda_{1}-\lambda_{n}\right)$, we know that

$$
\pi^{\prime}(\xi) q=r\left(2 a_{1}+a_{2}+\cdots+a_{n-1}\right) q .
$$


Since $a_{1}, \ldots, a_{n-1}$ may be arbitrary elements of $\mathbb{C}$, we deduce the relations

$$
r_{1}-r_{n}-s_{1}+s_{n}=2 r, \quad r_{j}-r_{n}-s_{j}+s_{n}=r,
$$

for $2 \leq j \leq n-1$. By considering the sum of all the equalities (5.3), we see that

$$
r_{1}+\cdots+r_{n-1}-\left(s_{1}+\cdots+s_{n-1}\right)-(n-1)\left(r_{n}-s_{n}\right)=n r .
$$

As we supposed that $r_{1}+\cdots+r_{n}=s_{1}+\cdots+s_{n}=r$, we obtain

$$
s_{n}=r_{n}+r \text {. }
$$

We deduce that $r_{n}=0$ and $s_{n}=r$. This last equality implies that $s_{j}=0$, for $1 \leq j \leq n-1$. The first equation of the system (5.3) tells us that $r_{1}=r$ and hence also that $r_{j}=0$, for $1 \leq j \leq n-1$.

By the methods which we just have used to prove Lemma 5.2 and by examining the action of the Cartan subalgebra $\mathfrak{h}$ on the appropriate weight vectors of $C^{\infty}\left(T_{\mathbb{C}}^{*}\right)$, we are also able to demonstrate the following lemma:

Lemma 5.3. Let $r \geq 1, r_{1}, \ldots, r_{n}, s_{1}, \ldots, s_{n} \geq 0$ be given integers; suppose that $r_{1}+\cdots+r_{n}=s_{1}+\cdots+s_{n}=r$. Let $q$ be the function $q_{r_{1}, \ldots, r_{n}, s_{1}, \ldots, s_{n}}$ and $\varphi$ be a 1 -form on $G$ equal to one of of the forms $\theta_{j k}$, with $1 \leq j<k \leq n$.

(i) The vector $q \varphi$ of $C^{\infty}\left(T_{\mathbb{C}}^{*}\right)$ is of weight $r\left(\lambda_{1}-\lambda_{n}\right)$ if and only if one of the following three assertions holds:

(a) the function $q$ is equal to $z_{n j} z_{n 1}^{r-1} \bar{z}_{1 n}^{r}$ and the 1 -form $\varphi$ is equal to $\theta_{1 j}$, where $2 \leq j \leq n$.

(b) the function $q$ is equal to $z_{n 1}^{r} \bar{z}_{1 k} \bar{z}_{1 n}^{r-1}$ and the 1 -form $\varphi$ is equal to $\theta_{k n}$, where $1 \leq k \leq n-1$.

(c) the function $q$ is equal to $z_{n j} \bar{z}_{1 j}\left(z_{n 1} \bar{z}_{1 n}\right)^{r-1}$ and the 1 -form $\varphi$ is equal to $\theta_{1 n}$, where $2 \leq j \leq n-1$.

(ii) For $1 \leq j<k \leq n$, the element $q \bar{\theta}_{j k}$ of $C^{\infty}\left(T_{\mathbb{C}}^{*}\right)$ is a weight vector whose weight is not equal to $r\left(\lambda_{1}-\lambda_{n}\right)$.

From Lemmas 4.2 and 5.3, we deduce the following result:

Proposition 5.4. Let $\gamma$ be the element $r\left(\varpi_{1}+\varpi_{n-1}\right)$ of $\Gamma$, where $r$ is a given integer $\geq 1$. The 1 -forms

$$
\left\{z_{n j} z_{n 1}^{r-1} \bar{z}_{1 n}^{r} \theta_{1 j}, z_{n 1}^{r} \bar{z}_{1 k} \bar{z}_{1 n}^{r-1} \theta_{k n}, z_{n s} \bar{z}_{1 s}\left(z_{n 1} \bar{z}_{1 n}\right)^{r-1} \theta_{1 n},\left(z_{n 1} \bar{z}_{1 n}\right)^{r} \omega_{l}\right\}
$$


on $G$, with $2 \leq j \leq n, 1 \leq k \leq n-1,2 \leq s \leq n-1$ and $1 \leq l \leq n-1$, generate the weight space $\mathcal{W}_{\gamma}$ of the $G$-submodule $\mathcal{C}_{\gamma} \cdot \mathcal{A}_{\mathbb{C}}$ of $C_{\gamma}^{\infty}\left(T_{\mathbb{C}}^{*}\right)$ corresponding to the weight $\gamma$.

Lemma 5.5. Let $\gamma$ be the element $r\left(\varpi_{1}+\varpi_{n-1}\right)$ of $\Gamma$, where $r$ is an integer $\geq 2$. Let $u$ be a highest weight vector of the $\tilde{G}$-module $C_{(\gamma, \gamma)}^{\infty}\left(T_{\mathbb{C}}^{*}\right)$ and $c$ be an element of $\mathbb{C}$ satisfying the relation

$$
D^{1} u=c \tilde{\sigma}^{\prime} d\left(z_{n 1} \bar{z}_{1 n}\right)^{r}
$$

Then the coefficient $c$ vanishes.

Proof. Throughout this proof, by $C_{l}$ and $A_{j k}$ we shall mean the left-invariant vector fields on $G$ determined by the corresponding elements of $\mathfrak{g}_{0}$. We write $f=z_{n 1} \bar{z}_{1 n}$. By means of (3.11), (2.8) and (2.10), we see that

$$
\begin{gathered}
\left(\tilde{\sigma}^{\prime}\left(d f^{r}\right)\right)\left(C_{1}, C_{1}\right)=-\left(\tilde{\sigma}^{\prime}\left(d f^{r}\right)\right)\left(C_{n-1}, C_{n-1}\right)=i r f^{r}, \\
\left(\tilde{\sigma}^{\prime}\left(d f^{r}\right)\right)\left(C_{k}, C_{l}\right)=0, \quad\left(2 \tilde{\sigma}^{\prime}\left(d f^{r}\right)\right)\left(C_{j}, A_{1 j}\right)=-r f^{r-1} z_{n j} \bar{z}_{1 n},
\end{gathered}
$$

for all $2 \leq j \leq n-1$ and $1 \leq k, l \leq n-1$, with $(k, l) \neq(1,1),(n-1, n-1)$. Since we know that $C_{(\gamma, \gamma)}^{\infty}\left(T_{\mathbb{C}}^{*}\right)$ is a subspace of $\mathcal{W}_{\gamma}$, according to Proposition 5.4 we may write

$$
\begin{aligned}
u=f^{r} & \sum_{l=1}^{n-1} p_{l} \omega_{l} \\
& +f^{r-1}\left(\sum_{j=2}^{n} a_{j} z_{n j} \bar{z}_{1 n} \theta_{1 j}+\sum_{k=1}^{n-1} b_{k} z_{n 1} \bar{z}_{1 k} \theta_{k n}+\sum_{l=2}^{n-1} c_{l} z_{n l} \bar{z}_{1 l} \theta_{1 n}\right),
\end{aligned}
$$

with $p_{l}, a_{j}, b_{k}, c_{l} \in \mathbb{C}$. By $(2.2)$ and (3.11), we obtain

$$
\begin{gathered}
\left(D^{1} u\right)\left(C_{1}, C_{1}\right)=\operatorname{irp}_{1} f^{r}, \quad\left(D^{1} u\right)\left(C_{n-1}, C_{n-1}\right)=i r p_{n-1} f^{r}, \\
\left(2 D^{1} u\right)\left(C_{1}, C_{l}\right)=i r p_{l} f^{r},
\end{gathered}
$$

for $2 \leq l \leq n-2$. According to (5.4) and (5.5) and our hypothesis, we see that

$$
p_{1}=-p_{n-1}=c, \quad p_{l}=0,
$$

for $2 \leq l \leq n-2$. By (2.2), (3.11) and the preceding equalities, we see that

$$
\begin{aligned}
& \left(2 D^{1} u\right)\left(C_{j}, A_{1 j}\right)=\left(i a_{j}+\delta_{j, n-1} r\left(c+i a_{j}\right)\right) f^{r-1} z_{n j} \bar{z}_{1 n}, \\
& \left(2 D^{1} u\right)\left(C_{1}, A_{12}\right)=\left(i(r-2) a_{2}-r c\right) f^{r-1} z_{n 2} \bar{z}_{1 n},
\end{aligned}
$$


for $2 \leq j \leq n-1$. According our hypothesis, from the relations (5.4) and (5.6), with $j=2$, we deduce that

$$
i a_{2}+\delta_{3 n} r\left(c+i a_{2}\right)=-r c
$$

on the other hand, by (2.9), with $j=1$, and (5.6), we also have

$$
i a_{2}(r-2)=r c .
$$

Hence we obtain

$$
i a_{2}(r-1)\left(1+2 \delta_{3 n}\right)=0 .
$$

Therefore since $r \geq 2$, the coefficient $a_{2}$ vanishes, and so does $c$.

Lemma 5.6. We have

$$
\tilde{\sigma}^{\prime} d \mathcal{B}^{\prime} \subset D^{1} C^{\infty}\left(T_{\mathbb{C}}^{*}\right)
$$

Proof. By Lemma 4.2, with $r=s=1$, the function $f=z_{n 1} \bar{z}_{1 n}$ is a highest weight vector of the irreducible $\tilde{G}$-module $\mathcal{B}^{\prime}$. According to Lemma 3.2 , the 2 -form $\tilde{\sigma}^{\prime} d f$ belongs to $D^{1} C^{\infty}\left(T_{\mathbb{C}}^{*}\right)$, and so we obtain the desired inclusion.

Lemma 5.7. Let $r \geq 2$ be a given integer and let $\gamma$ be the element $r\left(\varpi_{1}+\varpi_{n-1}\right)$ of $\Gamma$. Then we have

$$
D^{1} C_{\gamma}^{\infty}\left(T_{\mathbb{C}}^{*}\right) \cap \tilde{\sigma}^{\prime} d C_{\gamma}^{\infty}(X)=\{0\}
$$

Proof. According to Lemma 4.2, the function $\left(z_{n 1} \bar{z}_{1 n}\right)^{r}$ a highest weight vector of the irreducible $G$-module $\mathcal{C}_{\gamma}$. The lemma is a direct consequence of Proposition 5.1, with $p=2$, and Lemma 5.5.

\section{ISOSPECTRAL DEFORMATIONS OF THE SPECIAL UNITARY GROUP}

We consider the subset

$$
\Gamma^{\prime}=\left\{\gamma_{r_{1}, \ldots, r_{n-1}} \mid r_{1}, \ldots, r_{n-1} \in \mathbb{N}, \text { with } r_{1}+\cdots+r_{n-1}>0\right\}
$$

of $\Gamma$ and its subset

$$
\Gamma_{1}=\left\{\gamma_{r_{1}, \ldots, r_{n-1}} \in \Gamma^{\prime} \mid r_{1} r_{n-1}=0\right\} .
$$

Let $\Gamma_{2}$ be the subset of $\Gamma^{\prime}$ defined by

$$
\Gamma_{2}=\left\{\gamma_{r_{1}, \ldots, r_{n-1}} \in \Gamma^{\prime} \mid r_{n-2}=r_{n-1}=0 \text { or } r_{1}=r_{2}=0\right\}
$$


when $n \geq 4$ and by

$$
\Gamma_{2}=\left\{\gamma_{r_{1}, r_{2}} \in \Gamma^{\prime} \mid r_{1}, r_{2}>0 \text { and } r_{1} \neq r_{2}\right\}
$$

when $n=3$. By (4.3), if $\gamma \in \Gamma$ belongs to $\Gamma_{1}$ (resp. to $\Gamma_{2}$ ), then so does $\bar{\gamma}$. For $j=1,2$, the $G$-submodule $\mathcal{F}_{j}$ of $C^{\infty}(G)$ equal to the closure of the subspace

$$
\bigoplus_{\gamma \in \Gamma_{j}} C_{\gamma}^{\infty}(G)
$$

in $C^{\infty}(G)$ is infinite-dimensional. By (4.6), we know that $\mathcal{F}_{j}$ is invariant under conjugation; hence the $G$-module $\mathcal{F}_{j}$ is equal to the complexification of the infinite-dimensional $G$-submodule

$$
\mathcal{F}_{j, \mathbb{R}}=\left\{f \in \mathcal{F}_{j} \mid f=\bar{f}\right\}
$$

of $C_{\mathbb{R}}^{\infty}(G)$.

In this section, we suppose that the symmetric space $X$ is the group $G=$ $S U(n)$, with $n \geq 3$. Here we shall consider $C^{\infty}(G)$ and $C^{\infty}\left(S^{p} T_{\mathbb{C}}^{*}\right)$ endowed with their structures of $G$-module arising from the left action of $G$ on $X$. Most of this section is devoted to the proof of the following two propositions.

Proposition 6.1. Let $X$ be the symmetric space $S U(n)$, with $n \geq 3$. Let $p \geq 2$ be a given integer.

(i) If $p$ is an even integer, the relation (5.1) holds for all elements $\gamma$ of $\Gamma_{1}$.

(ii) If either $n \geq 4$ or $n=3$ and $p$ is an odd integer $\geq 5$, the relation (5.1) holds for all elements $\gamma$ of $\Gamma_{2}$.

Proposition 6.2. Let $X$ be the symmetric space $S U(n)$, with $n \geq 3$. We have

$$
D_{0} C^{\infty}(T) \cap \tilde{\sigma}^{\prime} d C_{\mathbb{R}}^{\infty}(X)=\tilde{\sigma}^{\prime} d \mathcal{B}_{\mathbb{R}}^{\prime}
$$

We consider the orthogonal complement $\mathcal{F}$ of the finite-dimensional subspace $\mathcal{F}^{\prime}=\mathbb{R}(G) \oplus \mathcal{B}_{\mathbb{R}}^{\prime}$ of $C_{\mathbb{R}}^{\infty}(G)$. According to Lemma 2.3, we know that the mapping

$$
P_{\sigma^{\prime}}=P \tilde{\sigma}^{\prime} d: C_{\mathbb{R}}^{\infty}(X) \rightarrow I(X)
$$

is well-defined. Proposition 6.2 tells us that the kernel of $P_{\sigma^{\prime}}$ is the finitedimensional space $\mathcal{F}^{\prime}$ and that the mapping $P_{\sigma^{\prime}}: \mathcal{F} \rightarrow I(X)$ is injective.

From Proposition 6.1, we deduce 
Proposition 6.3. Let $X$ be the symmetric space $S U(n)$, with $n \geq 3$. Let $p \geq 2$ be a given integer.

(i) If $p$ is an even integer, we have

$$
D^{p-1} C^{\infty}\left(S^{p-1} T^{*}\right) \cap \tilde{\sigma}_{p+1}^{\prime} d \mathcal{F}_{1, \mathbb{R}}=\{0\} .
$$

(ii) If either $n \geq 4$ or $n=3$ and $p$ is an odd integer $\geq 5$, we have

$$
D^{p-1} C^{\infty}\left(S^{p-1} T^{*}\right) \cap \tilde{\sigma}_{p+1}^{\prime} d \mathcal{F}_{2, \mathbb{R}}=\{0\} .
$$

For the proof of Proposition 6.3,(ii), with $n=3$, we shall require the following lemma:

Lemma 6.4. Let $p \geq 5$ be a given odd integer and let $a, b>0$ be given real numbers satisfying $a \neq b$. Let $\left\{\varepsilon_{1}, \ldots, \varepsilon_{p+1}\right\}$ be the standard basis of $\mathbb{R}^{p+1}$. The vectors $\left\{\xi_{1}, \ldots, \xi_{p+1}\right\}$ of $\mathbb{R}^{p+1}$ given by

$$
\begin{gathered}
\xi_{1}=p \varepsilon_{1}-3 \varepsilon_{p+1}, \quad \xi_{p}=p \varepsilon_{p}-3 \varepsilon_{p+1}, \\
\xi_{k}=(k-1) b \varepsilon_{k-1}+(p-k+1) a \varepsilon_{k}+(-1)^{k}(a-b) \varepsilon_{p+1},
\end{gathered}
$$

for $2 \leq k \leq p$, form a basis of $\mathbb{R}^{p+1}$.

Proof. Let $c_{1}, \ldots, c_{p+1}$ be real numbers and consider the vector

$$
\xi=c_{1} \xi_{1}+\cdots+c_{p+1} \xi_{p+1}
$$

of $\mathbb{R}^{p+1}$. Then we have $\xi=v_{1} \varepsilon_{1}+\cdots+v_{p+1} \varepsilon_{p+1}$, where the coefficients $v_{1}, \ldots, v_{p+1}$ are given by

$$
\begin{gathered}
v_{1}=p c_{1}+b c_{2}, \quad v_{k}=(p-k+1) a c_{k}+k b c_{k+1}, \quad v_{p}=a c_{p}+p c_{p+1}, \\
v_{p+1}=-3\left(c_{1}+c_{p+1}\right)+(a-b) \sum_{k=2}^{p}(-1)^{k} c_{k},
\end{gathered}
$$

for $2 \leq k \leq p$. We now suppose that $\xi$ vanishes. From the previous formulas, we deduce that

$$
b^{k-1} c_{k}=(-1)^{k-1}\left(\begin{array}{c}
p \\
k-1
\end{array}\right) a^{k-2} c_{1}, \quad b^{p-1} c_{p+1}=-a^{p-1} c_{1},
$$

for $2 \leq k \leq p$; then we obtain

$$
b^{p-1} v_{p+1}=\left(3\left(a^{p-1}-b^{p-1}\right)-(a-b) \sum_{k=2}^{p}\left(\begin{array}{c}
p \\
k-1
\end{array}\right) a^{k-2} b^{p-k}\right) c_{1} .
$$


Since $p \geq 5$, we know that the integer

$$
u_{k}=3-\left(\begin{array}{l}
p \\
k
\end{array}\right)
$$

is negative, for $1 \leq k \leq p$; from the equality (6.2), it follows that

$$
a b^{p} v_{p+1}=c_{1}(a-b) \sum_{k=1}^{p-1} u_{k} a^{p-k} b^{k} .
$$

Our hypothesis says that $v_{p+1}$ vanishes and so the previous equation implies that $c_{1}$ also vanishes. According to the formulas (6.1), it follows that $c_{k}=0$, for $2 \leq k \leq p+1$. Thus the vectors $\left\{\xi_{1}, \ldots, \xi_{p+1}\right\}$ are linearly independent.

Throughout this section, by $C_{j}, \tilde{C}_{l}$ and $A_{k l}$ we shall always mean the leftinvariant vector fields on $G$ determined by the corresponding elements of $\mathfrak{g}_{0}$.

Let $r_{1}, \ldots, r_{n-1} \geq 0$ be given integers which are not all zero, and let $\gamma$ be the element $\gamma_{r_{1}, \ldots, r_{n-1}}$ of $\Gamma$. We set

$$
d_{k}=\frac{1}{n}\left(\sum_{j=k}^{n-1}(n-j) r_{j}-\sum_{j=1}^{k-1} j r_{j}\right),
$$

for $1 \leq k \leq n$. We note that the sum $d_{n-1}+d_{n}$ is always negative (see $[5, \S 8]$ ).

Let $f$ be a highest weight vector of the $G$-module $C_{\gamma}^{\infty}(G)$. Then by (4.2) and (4.5), we have

$$
\left(C_{l} f\right)\left(e_{0}\right)=-i r_{l} f\left(e_{0}\right), \quad\left(\tilde{C}_{j} f\right)\left(e_{0}\right)=-i d_{j} f\left(e_{0}\right),
$$

for all $1 \leq l \leq n-1$ and $1 \leq j \leq n$.

Lemma 6.5. Let $p \geq 2$ be a given integer. Let $r_{1}, \ldots, r_{n-1} \geq 0$ be given integers which are not all zero and let $\gamma$ be the element $\gamma_{r_{1}, \ldots, r_{n-1}}$ of $\Gamma^{\prime}$. Suppose that one of the following conditions holds:

(i) $p$ is an even integer and $r_{n-1}=0$;

(ii) $n \geq 4$ and $r_{n-2}=r_{n-1}=0$;

(iii) $p=2$ and $\left(r_{1}, \ldots, r_{n-1}\right) \neq(r, 0, \ldots, 0, r)$, with $r \geq 1$;

(iv) $n$ is equal to 3 and $p$ is an odd integer $\geq 5$, and $r_{1}, r_{2}$ are distinct positive integers. 
Let $f$ be a highest weight vector of the $G$-module $C_{\gamma}^{\infty}(G)$. Let $u$ be an element of the subspace $\mathcal{C}_{\gamma} \cdot S^{p-1} \mathcal{A}_{\mathbb{C}}$ of $C^{\infty}\left(S^{p-1} T_{\mathbb{C}}^{*}\right)$ and $c$ be an element of $\mathbb{C}$ satisfying the relation

$$
p D^{p-1} u=c \tilde{\sigma}_{p+1}^{\prime}(d f) .
$$

Then the coefficient $c$ vanishes.

Proof. There exists an element $a \in G$ such that $f(a) \neq 0$; we denote by $R_{a}$ the right action of the element $a$ on $G$. Replacing $f$ by $R_{a}^{*} f$, if necessary, we may assume that $f\left(e_{0}\right) \neq 0$. We choose a basis $\left\{f_{1}, \ldots, f_{q}\right\}$ of $\mathcal{C}_{\gamma}$, with $f_{1}=f$ and $f_{s}\left(e_{0}\right)=0$, for all $2 \leq s \leq q$. Then the element $u$ of $C^{\infty}\left(S^{p-1} T_{\mathbb{C}}^{*}\right)$ can be written in the form

$$
u=\sum_{s=1}^{q} f_{s} u_{s}
$$

where $u_{1}, \ldots, u_{q}$ are elements of $S^{p-1} \mathcal{A}_{\mathbb{C}}$. From (6.4) and (2.2), we obtain the relation

$$
\sum_{s=1}^{q} d f_{s} \cdot u_{s}=c \tilde{\sigma}_{p+1}^{\prime}(d f) .
$$

We first suppose that condition (i) holds. For $1 \leq s \leq q$, by Lemma 5.1,(i) of [5], we know that $\pi\left(A_{n-1, n}\right) f_{s}=0$. Then by (4.5), we know that $\left(A_{n-1, n} f_{s}\right)\left(e_{0}\right)=0$; thus the relation

$$
\left(d f_{s} \cdot u_{s}\right)\left(A_{n-1, n}, \ldots, A_{n-1, n}\right)=0
$$

holds at $e_{0}$. According to (2.6) and (6.3), the equality

$$
\tilde{\sigma}_{p+1}^{\prime}(d f)\left(A_{n-1, n}, \ldots, A_{n-1, n}\right)=-i\left(d_{n-1}+d_{n}\right) f
$$

holds at $e_{0}$. As we remarked above, the number $d_{n-1}+d_{n}$ is negative. Therefore the equalities (6.5)-(6.7) imply that the coefficient $c$ vanishes. Next, we suppose that condition (ii) holds. For $1 \leq s \leq q$, by (6.3) we know that

$$
\left(C_{n-2} f_{s}\right)\left(e_{0}\right)=\left(C_{n-1} f_{s}\right)\left(e_{0}\right)=0 ;
$$

thus the relation

$$
\left(d f_{s} \cdot u_{s}\right)\left(C_{n-2}, C_{n-1}, \ldots, C_{n-1}\right)=0
$$

holds at $e_{0}$. According to (2.5) and (6.3), the equality

$$
\tilde{\sigma}_{p+1}^{\prime}(d f)\left(C_{n-2}, C_{n-1}, \ldots, C_{n-1}\right)=i d_{n-1} f
$$


holds at $e_{0}$. Under our hypotheses, we have

$$
d_{n-1}=-\sum_{j=1}^{n-3} j r_{j}
$$

and so the number $d_{n-1}$ is negative. Therefore the equalities (6.5), (6.8) and (6.9) imply that the coefficient $c$ vanishes. Next, we suppose that condition (iii) holds. We may write

$$
u_{1}=\sum_{j=1}^{n-1} a_{j} \omega_{j}+u^{\prime},
$$

where $a_{j} \in \mathbb{C}$ and $u^{\prime}$ is an element of $\mathcal{A}_{\mathbb{C}}$ satisfying $u^{\prime}\left(C_{j}\right)=0$, for all $1 \leq j \leq n-1$. Then by (6.3), for all $1 \leq j, k \leq n-1$ and $2 \leq s \leq q$, the equalities

$$
\left(d f_{1} \cdot u_{1}\right)\left(C_{j}, C_{k}\right)=-i\left(a_{j} r_{k}+a_{k} r_{j}\right) f, \quad\left(d f_{s} \cdot u_{s}\right)\left(C_{j}, C_{k}\right)=0
$$

hold at the point $e_{0} \in G$. By (2.3)-(2.5) and (6.3), we see that

$$
\tilde{\sigma}_{3}^{\prime}(d f)\left(C_{j}, C_{k}\right)\left(e_{0}\right)=0,
$$

for all $1 \leq j, k \leq n-1$, whenever $k \geq j+2$, and that the equalities

$$
\tilde{\sigma}_{3}^{\prime}(d f)\left(C_{j}, C_{j}\right)=-i\left(d_{j}+d_{j+1}\right) f, \quad \tilde{\sigma}_{3}^{\prime}(d f)\left(C_{k}, C_{k+1}\right)=i d_{k+1} f
$$

hold at $e_{0}$, for all $1 \leq j \leq n-1$ and $1 \leq k \leq n-2$. Since $f\left(e_{0}\right) \neq 0$, the integers $r_{1}, \ldots, r_{n-1}$ and the complex numbers $a_{1}, \ldots, a_{n-1}$ satisfy the hypotheses of Proposition 8.1 of [5]. Now Proposition 8.1,(i) of [5] gives us the vanishing of the constant $c$. Finally, we suppose that condition (iv) holds. We may write

$$
u_{1}=\sum_{k=0}^{p-1} \frac{a_{k}}{k !(p-k-1) !} \omega_{1}^{k} \cdot \omega_{2}^{p-k-1}+u^{\prime},
$$

where $a_{0}, \ldots, a_{p-1} \in \mathbb{C}$ and $u^{\prime}$ is an element of $S^{p-1} \mathcal{A}_{\mathbb{C}}$ satisfying

$$
u^{\prime}\left(C_{1}, \ldots, C_{1}, C_{2}, \ldots, C_{2}\right)=0
$$

if the vector field $C_{1}$ appears $k$ times in the left-hand side of the last equation, with $0 \leq k \leq p-1$. If $\varphi$ is an element of $T_{e_{0}}^{*}$, we easily verify that

$$
\begin{gathered}
\left(\varphi \cdot u_{1}\right)\left(C_{1}, \ldots, C_{1}\right)=p a_{p-1} \varphi\left(C_{1}\right), \quad\left(\varphi \cdot u_{1}\right)\left(C_{2}, \ldots, C_{2}\right)=p a_{0} \varphi\left(C_{2}\right), \\
\left(\varphi \cdot u_{1}\right)\left(C_{1}, \ldots, C_{1}, C_{2}, \ldots, C_{2}\right)=k a_{k-1} \varphi\left(C_{1}\right)+(p-k) a_{k} \varphi\left(C_{2}\right),
\end{gathered}
$$


if the vector field $C_{1}$ appears $k$ times in the left-hand side of the last equation, with $1 \leq k \leq p-1$. Also according to (2.4) and (2.5), for $j=1,2$, the equalities

$$
\begin{aligned}
\tilde{\sigma}_{p+1}^{\prime}(\varphi)\left(C_{j}, \ldots, C_{j}\right) & =\varphi\left(C_{j}\right) \\
\tilde{\sigma}_{p+1}^{\prime}(\varphi)\left(C_{1}, \ldots, C_{1}, C_{2}, \ldots, C_{2}\right) & =\frac{(-1)^{k}}{3} \varphi\left(C_{2}-C_{1}\right)
\end{aligned}
$$

hold at $e_{0}$, if the vector field $C_{1}$ appears $k$ times in the left-hand side of the last equation, with $1 \leq k \leq p-1$. For $2 \leq s \leq q$, by (6.3) the equality

$$
\left(d f_{s} \cdot u_{s}\right)\left(C_{1}, \ldots, C_{1}, C_{2}, \ldots, C_{2}\right)=0
$$

holds at the point $e_{0}$, if the vector field $C_{1}$ appears $k$ times in the left-hand side of this equation, with $0 \leq k \leq p$. Since $f\left(e_{0}\right)$ is non-zero, from the relations (6.3) and (6.5) and the preceding equalities we obtain

$$
\begin{gathered}
p a_{p-1} r_{1}=c r_{1}, \quad p a_{0} r_{2}=c r_{2}, \\
(p-k) r_{1} a_{p-k-1}+k r_{2} a_{p-k}=(-1)^{k+1} c^{\prime}\left(r_{2}-r_{1}\right),
\end{gathered}
$$

for $1 \leq k \leq p-1$, where $c^{\prime}=c / 3$. If $\left\{\xi_{1}, \ldots, \xi_{p+1}\right\}$ are the vectors of $\mathbb{R}^{p+1}$ associated in Lemma 6.4 with the integer $p$ and the positive real numbers $a=r_{1}$ and $b=r_{2}$, the previous equalities say that the vector $v=\left(a_{p-1}, \ldots, a_{1}, a_{0}, c^{\prime}\right)$ of $\mathbb{R}^{p+1}$ is orthogonal to $\xi_{k}$ (with respect to the standard Euclidean scalar product on $\mathbb{R}^{p+1}$ ), for all $1 \leq k \leq p+1$. Since the integers $r_{1}$ and $r_{2}$ are distinct positive integers, by Lemma 6.4 the vector $v$ vanishes and hence so does $c$.

Let $p \geq 2$ be a given integer. We remind the reader that the weight space $\mathcal{C}_{(\gamma, \bar{\gamma})}\left(S^{p-1} T_{\mathbb{C}}^{*}\right)$ is a subspace of $\mathcal{C}_{\gamma} \cdot S^{p-1} \mathcal{A}_{\mathbb{C}}$. We now begin our proof of Proposition 6.1. Let $r_{1}, \ldots, r_{n-1} \geq 0$ be given integers, which are not all zero, and $\gamma$ be the element $\gamma_{r_{1}, \ldots, r_{n-1}}$ of $\Gamma^{\prime}$. If condition (i), (ii) or (iv) of Lemma 6.5 holds, the equality (5.1) for $\gamma$ is a consequence of Proposition 5.1 and Lemma 6.5; by means of the relation (4.6), we see that the equality (5.1) also holds for $\bar{\gamma}$. Thus by (4.3), we know that (5.1) is true when $p$ is even and $r_{1}=0$, or when $n \geq 4$ and $r_{1}=r_{2}=0$. This completes the proof of Proposition 6.1.

We now turn to the proof of Proposition 6.2. By formula (1.2), to prove this proposition it suffices to show that

$$
D^{1} C^{\infty}\left(T_{\mathbb{C}}^{*}\right) \cap \tilde{\sigma}^{\prime} d C^{\infty}(X)=\tilde{\sigma}^{\prime} d \mathcal{B}^{\prime} .
$$


Since the differential operators $D^{1}$ and $\tilde{\sigma}^{\prime} d$ are homogeneous, according to Proposition 2.1 of [3] and Lemma 5.6 the preceding equality holds if and only if the relation (5.7) holds for all $\gamma \in \Gamma$, with $\gamma \neq \gamma_{1,0, \ldots, 0,1}$. If $\gamma \in \Gamma$ is equal to 0 , we have $d C_{\gamma}^{\infty}(X)=\{0\}$, and so the equality (5.7) holds. Let $r_{1}, \ldots, r_{n-1} \geq 0$ be given integers, which are not all zero and satisfy

$$
\left(r_{1}, \ldots, r_{n-1}\right) \neq(1,0, \ldots, 0,1)
$$

and consider the element $\gamma=\gamma_{r_{1}, \ldots, r_{n-1}}$ of $\Gamma^{\prime}$. When $\gamma \neq \gamma_{r, 0, \ldots, 0, r}$, with $r \geq 2$, the relation (5.7) is a direct consequence of Proposition 5.1, with $p=2$, and Lemma 6.5,(iii). When $\gamma=\gamma_{r, 0, \ldots, 0, r}$, with $r \geq 2$, the equality (5.7) is given by Lemma 5.7. Therefore the equality (5.7) holds for all $\gamma \in \Gamma$, with $\gamma \neq \gamma_{1,0, \ldots, 0,1}$, and so we have completed the proof of Proposition 6.2.

When $n=3$, according to Lemma 2.2, we have the inclusion

$$
\tilde{\sigma}_{4}^{\prime} d C_{\mathbb{R}}^{\infty}(X) \subset D^{2} C^{\infty}\left(T^{*}\right) .
$$

Therefore when $n=p=3$, if $\gamma$ is an arbitrary element of $\Gamma^{\prime}$, the relation (5.1) does not hold, and the assertion of Lemma 6.5 is not true.

\section{THE REDUCED UNITARY GROUP}

The center of $G=S U(n)$ is the cyclic subgroup $S$ of order $n$ equal to

$$
\left\{e^{2 i k \pi / n} I_{n} \mid 0 \leq k \leq n-1\right\} .
$$

If $E$ is a $G$-module, we denote by $E^{S}$ the $G$-submodule of $E$ consisting of all $S$-invariant elements of $E$. Let $\check{\Gamma}$ be the subset of $\Gamma$ consisting of all elements $\gamma_{r_{1}, \ldots, r_{n-1}}$ of $\Gamma$, where $r_{1}, \ldots, r_{n-1}$ are non-negative integers satisfying the relation

$$
r_{1}+2 r_{2}+\cdots+(n-1) r_{n-1} \equiv 0 \bmod n .
$$

If $\gamma$ is an element of $\Gamma$, from Lemma 5.1,(ii) of [5] we deduce that $C_{\gamma}^{\infty}(G)$ is a $G$-submodule of $C^{\infty}(G)^{S}$ if and only if $\gamma$ belongs to $\check{\Gamma}$.

The group $\check{G}=G / S$ is a symmetric space of compact type, which is the reduced space of the symmetric space $G$ and which we call the reduced unitary group; it is isomorphic to the adjoint group of $\mathfrak{s u}(n)$ (see $\S 9$, Chapter VII of [8]). In this section, we suppose that the symmetric space $X$ is the group $\breve{G}$. 
We consider the natural projection $\pi: G \rightarrow \check{G}$. If $\gamma$ is an element of $\Gamma$, we know that the isomorphism $\pi^{*}: C^{\infty}(\breve{G}) \rightarrow C^{\infty}(G)^{S}$ induces an isomorphism of $G$-modules

$$
\pi^{*}: C_{\gamma}^{\infty}(\check{G}) \rightarrow C_{\gamma}^{\infty}(G)^{S}
$$

of $G$-modules; according to Lemma 5.1,(ii) of [5], we know that

$$
C_{\gamma}^{\infty}(G)^{S}=C_{\gamma}^{\infty}(G)
$$

if and only if $\gamma$ belongs to $\check{\Gamma}$.

We consider the element $\gamma=\varpi_{1}+\varpi_{n-1}$ of $\check{\Gamma}$. We just saw that

$$
\mathcal{B}^{\prime S}=\mathcal{B}^{\prime}
$$

Therefore $\check{\mathcal{B}}=C_{\gamma}^{\infty}(\check{G})$ is isomorphic to $\mathcal{B}^{\prime}$ and invariant under conjugation; thus $\check{\mathcal{B}}$ is equal to the complexification of the subspace

$$
\check{\mathcal{B}}_{\mathbb{R}}=\{f \in \check{\mathcal{B}} \mid f=\bar{f}\}
$$

of $C_{\mathbb{R}}^{\infty}(\check{G})$ and the mapping $\pi$ induces an isomorphism $\pi^{*}: \check{\mathcal{B}}_{\mathbb{R}} \rightarrow \mathcal{B}_{\mathbb{R}}^{\prime}$.

For $j=1,2$, we consider the subset $\check{\Gamma}_{j}=\Gamma_{j} \cap \check{\Gamma}$ of $\Gamma$; let $\check{\mathcal{F}}_{j}$ denote the $G$-submodule of $C^{\infty}(\check{G})$ equal to the closure of the subspace

$$
\bigoplus_{\gamma \in \check{\Gamma}_{j}} C_{\gamma}^{\infty}(\check{G})
$$

in $C^{\infty}(\check{G})$. If $f$ is an element of $C^{\infty}(\check{G})$, then $f$ belongs to $\check{\mathcal{F}}_{j}$ if and only if $\pi^{*} f \in \mathcal{F}_{j}$; thus the $G$-module $\check{\mathcal{F}}_{j}$ is equal to the complexification of the infinitedimensional $G$-submodule

$$
\check{\mathcal{F}}_{j, \mathbb{R}}=\left\{f \in \check{\mathcal{F}}_{j} \mid f=\bar{f}\right\}
$$

of $C_{\mathbb{R}}^{\infty}(\check{G})$.

For $p \geq 2$, the $G$-invariant symmetric $p$-form $\sigma_{p}^{\prime}$ on $G$ induces a $G$-invariant symmetric $p$-form $\sigma_{p}^{\prime \prime}$ on $\breve{G}$ satisfying

$$
\pi^{*} \sigma_{p}^{\prime \prime}=\sigma_{p}^{\prime}
$$

We endow the symmetric space $X=\check{G}$ with the metric $\sigma_{2}^{\prime \prime}$. For $p \geq 3$, we consider the monomorphism of vector bundles

$$
\tilde{\sigma}_{p}^{\prime \prime}: T^{*} \rightarrow S^{p-1} T^{*}
$$


induced by the symmetric $p$-form $\sigma_{p}^{\prime \prime}$. We write $\sigma^{\prime \prime}=\sigma_{3}^{\prime \prime}$ and $\tilde{\sigma}^{\prime \prime}=\tilde{\sigma}_{3}^{\prime \prime}$. If $\varphi$ is a 1-form on $\breve{G}$, we have

$$
\pi^{*} \tilde{\sigma}_{p}^{\prime \prime}(\varphi)=\tilde{\sigma}_{p}^{\prime}\left(\pi^{*} \varphi\right)
$$

According to Lemma 1.1 of [4] and Lemma 2.3, for all $p \geq 2$, we see that a 1-form $\varphi$ on $\check{G}$ satisfies the Guillemin condition if and only if the symmetric $p$-form $\tilde{\sigma}_{p+1}^{\prime \prime}(\varphi)$ on $\breve{G}$ satisfies the Guillemin condition.

If $p \geq 2$ is a given integer and $\gamma$ is an element of $\Gamma$, we consider the relation

$$
D^{p-1} C_{\gamma}^{\infty}\left(S^{p-1} T_{\mathbb{C}}^{*}\right) \cap \tilde{\sigma}_{p+1}^{\prime \prime} d C_{\gamma}^{\infty}(X)=\{0\} .
$$

By Proposition 6.1 and the relation (7.2), we obtain the following result:

Proposition 7.1. Let $X$ be the symmetric space $S U(n) / S$, with $n \geq 3$. Let $p \geq 2$ be a given integer.

(i) If $p$ is an even integer, the relation (7.3) holds for all elements $\gamma$ of $\check{\Gamma}_{1}$.

(ii) If either $n \geq 4$ or $n=3$ and $p$ is an odd integer $\geq 5$, the relation (7.3) holds for all elements $\gamma$ of $\check{\Gamma}_{2}$.

From Proposition 7.1, we deduce

Proposition 7.2. Let $X$ be the symmetric space $S U(n) / S$, with $n \geq 3$. Let $p \geq 2$ be a given integer.

(i) If $p$ is an even integer, we have

$$
D^{p-1} C^{\infty}\left(S^{p-1} T^{*}\right) \cap \tilde{\sigma}_{p+1}^{\prime \prime} d \check{\mathcal{F}}_{1, \mathbb{R}}=\{0\} .
$$

(ii) If either $n \geq 4$ or $n=3$ and $p$ is an odd integer $\geq 5$, we have

$$
D^{p-1} C^{\infty}\left(S^{p-1} T^{*}\right) \cap \tilde{\sigma}_{p+1}^{\prime \prime} d \check{\mathcal{F}}_{2, \mathbb{R}}=\{0\} .
$$

From Proposition 6.2 and the relation (7.2), we obtain the following result:

Proposition 7.3. Let $X$ be the symmetric space $S U(n) / S$, with $n \geq 3$. We have

$$
D_{0} C^{\infty}(T) \cap \tilde{\sigma}^{\prime \prime} d C_{\mathbb{R}}^{\infty}(X)=\tilde{\sigma}^{\prime \prime} d \check{\mathcal{B}}_{\mathbb{R}} .
$$

If $P$ denotes the orthogonal projection corresponding to the decomposition (1.5) on the space $Y$, according to Lemma 1.1 of [4] and Lemma 2.3 the mapping

$$
P_{\sigma^{\prime \prime}}=P \tilde{\sigma}^{\prime \prime} d: C_{\mathbb{R}}^{\infty}(X) \rightarrow I(X)
$$


is well-defined. We denote by $\check{\mathcal{F}}$ the orthogonal complement of the finite-dimensional space $\check{\mathcal{F}}^{\prime}=\mathbb{R}(\check{G}) \oplus \check{\mathcal{B}}_{\mathbb{R}}$ in $C_{\mathbb{R}}^{\infty}(\check{G})$. From Proposition 1.2 of [4] and Proposition 7.3, we obtain:

Theorem 7.4. The reduced unitary group $X=\check{G}=S U(n) / S$ is not rigid in the sense of Guillemin. If $f$ is a non-zero element of $\check{\mathcal{F}}$, then the symmetric 2-form $\tilde{\sigma}^{\prime \prime}(d f)$ on $X$ satisfies the Guillemin condition and is not a Lie derivative of the metric. Moreover, the kernel of the mapping (7.4) is the finite-dimensional space $\mathbb{R}(X) \oplus \check{\mathcal{B}}_{\mathbb{R}}$.

According to the observations made in $\S 1$, we see that Proposition 7.2 implies that the equality (1.6), with $p \geq 2$, does not hold when $n \geq 4$ or $p$ is even. Thus we have proved the following result:

Theorem 7.5. Let $X$ be the symmetric space $S U(n) / S$, with $n \geq 3$, and let $p \geq 2$ be a given integer. The equality (1.6) does not hold whenever $(n, p) \neq(3,3)$. If $f$ is a non-zero real-valued function on $X$ which belongs to $\check{\mathcal{F}}_{1, \mathbb{R}}\left(\right.$ resp. to $\left.\check{\mathcal{F}}_{2, \mathbb{R}}\right)$ and $p$ is an even integer (resp. $n \geq 4$ or $n=3$ and $p$ is an odd integer $\geq 5$ ), then the symmetric $p$-form $\tilde{\sigma}_{p+1}^{\prime \prime}(d f)$ on $X$ satisfies the Guillemin condition and does not belong to the space $D^{p-1} C^{\infty}\left(S^{p-1} T^{*}\right)$.

\section{CHAPTER II: The SYMMETRIC SPACE $S U(2 n) / S p(n)$}

\section{Branching LAWS AND the DECOMPOSition of A SPACE of TENSORS}

If $V$ is a complex finite-dimensional vector space, we denote by $\bigotimes^{k} V, S^{l} V$ and $\bigwedge^{j} V$ the $k$-th tensor product, the $l$-th symmetric product and the $j$-th exterior product of $V$, respectively; we shall identify $S^{k} V^{*}$ and $\bigwedge^{k} V^{*}$ with subspaces of $\otimes^{k} V^{*}$. Let $n \geq 3$ be a given integer and let $U$ be a complex vector space of dimension $2 n$ endowed with a non-degenerate skew-symmetric bilinear form $q$. We consider the group $S L(U)$ consisting of all automorphisms of $U$ whose determinants are equal to 1 and its subgroup $S p(U)$ consisting of those elements of $S L(U)$ which preserve the bilinear form $q$.

Let $B(U)$ be the subspace of $\bigwedge^{2} U^{*} \otimes \bigwedge^{2} U^{*}$ consisting of those elements $v$ of $\bigwedge^{2} U^{*} \otimes \bigwedge^{2} U^{*}$ which satisfy the first Bianchi identity

$$
v\left(\xi_{1}, \xi_{2}, \xi_{3}, \xi_{4}\right)+v\left(\xi_{2}, \xi_{3}, \xi_{1}, \xi_{4}\right)+v\left(\xi_{3}, \xi_{1}, \xi_{2}, \xi_{4}\right)=0,
$$


for all $\xi_{1}, \xi_{2}, \xi_{3}, \xi_{4} \in U$; it is well-known that $B(U)$ is an irreducible $S L(U)$ submodule of $S^{2}\left(\bigwedge^{2} U^{*}\right)$. The natural monomorphism

$$
\bigwedge^{p} U^{*} \rightarrow \bigwedge^{2} U^{*} \otimes \bigwedge^{p-2} U^{*}
$$

allows us to view $\bigwedge^{p} U^{*}$ as an irreducible $S L(U)$-module of $\bigwedge^{2} U^{*} \otimes \bigwedge^{p-2} U^{*}$. We view $\bigwedge^{4} U^{*}$ is an irreducible $S L(U)$-submodule of $S^{2}\left(\bigwedge^{2} U^{*}\right)$. In fact, it is well-known that $S^{2}\left(\bigwedge^{2} U^{*}\right)$ admits the decomposition

$$
S^{2}\left(\bigwedge^{2} U^{*}\right)=B \oplus \bigwedge^{4} U^{*}
$$

Let $1 \leq k \leq n$ be a given integer. Let $\mathfrak{S}_{2 k}$ be the group of permutations of $\{1,2, \ldots, 2 k\}$; we denote by $\operatorname{sgn} \tau$ the signature of an element $\tau$ of $\mathfrak{S}_{2 k}$. The element $q^{k}$ of $\bigwedge^{2 k} U^{*}$, which is the $k$-th power of $q$, satisfies

$$
q^{k}\left(\xi_{1}, \ldots, \xi_{2 k}\right)=\frac{1}{2^{k}} \sum_{\tau \in \mathfrak{S}_{2 k}} \operatorname{sgn} \tau \cdot \prod_{j=1}^{k} q\left(\xi_{\tau(2 j-1)}, \xi_{\tau(2 j)}\right),
$$

for all $\xi_{1}, \ldots, \xi_{2 k} \in U$.

If $q^{b}: U \rightarrow U^{*}$ is the natural isomorphism determined by $q$ and given by

$$
\left\langle\eta, q^{b}(\xi)\right\rangle=q(\xi, \eta)
$$

for all $\xi, \eta \in U$, we define a mapping $\tau: \bigotimes^{2} U^{*} \rightarrow \mathbb{C}$ by

$$
\tau\left(q^{b}\left(\xi_{1}\right) \otimes q^{b}\left(\xi_{2}\right)\right)=q\left(\xi_{1}, \xi_{2}\right),
$$

for all $\xi_{1}, \xi_{2} \in U$. Let $\left\{v_{1}, \ldots, v_{n}, w_{1}, \ldots, w_{n}\right\}$ be a basis of $U$ satisfying

$$
q\left(v_{j}, v_{k}\right)=q\left(w_{j}, w_{k}\right)=0, \quad q\left(v_{j}, w_{k}\right)=\delta_{j k},
$$

for $1 \leq j, k \leq n$; we easily see that

$$
\tau(\beta)=\sum_{j=1}^{n}\left(\beta\left(v_{j}, w_{j}\right)-\beta\left(w_{j}, v_{j}\right)\right),
$$

for all $\beta \in \bigotimes^{2} U^{*}$; it follows that $\tau(q)=2 n$.

The restriction

$$
\tau: \bigwedge^{p} U^{*} \rightarrow \bigwedge^{p-2} U^{*}
$$

of the morphism

$$
\tau \otimes \mathrm{id}: \bigwedge^{2} U^{*} \otimes \bigwedge^{p-2} U^{*} \rightarrow \bigwedge^{p-2} U^{*}
$$


to $\bigwedge^{p} U^{*}$ is an epimorphism of $S p(U)$-modules; for $1 \leq p \leq n$, its kernel $\bigwedge_{0}^{p} U^{*}$ is an irreducible $S p(U)$-module (see [2, Theorem 17.5]). The one-dimensional subspace $\{q\}$ of $\bigwedge^{2} U^{*}$ generated by $q$ is a trivial $S p(U)$-module and we have the decomposition

$$
\bigwedge^{2} U^{*}=\bigwedge_{0}^{2} U^{*} \oplus\{q\}
$$

of $\bigwedge^{2} U^{*}$ into irreducible $S p(U)$-modules. We also see that $\bigwedge_{0}^{4} U^{*}$ is an irreducible $S p(U)$-submodule of $S^{2}\left(\bigwedge_{0}^{2} U^{*}\right)$.

We denote by $\tau_{1}$ the morphism (8.3) with $p=4$ and we consider the morphism

$$
\phi: \bigwedge^{2} U^{*} \rightarrow S^{2}\left(\bigwedge^{2} U^{*}\right)
$$

of $S p(U)$-modules defined by

$$
\begin{aligned}
(\phi u)\left(\xi_{1}, \xi_{2}, \xi_{3}, \xi_{4}\right)=n( & q\left(\xi_{1}, \xi_{4}\right) u\left(\xi_{2}, \xi_{3}\right)-q\left(\xi_{2}, \xi_{4}\right) u\left(\xi_{1}, \xi_{3}\right) \\
& \left.-q\left(\xi_{1}, \xi_{3}\right) u\left(\xi_{2}, \xi_{4}\right)+q\left(\xi_{2}, \xi_{3}\right) u\left(\xi_{1}, \xi_{4}\right)\right) \\
+ & 2\left(q\left(\xi_{1}, \xi_{2}\right) u\left(\xi_{3}, \xi_{4}\right)+q\left(\xi_{3}, \xi_{4}\right) u\left(\xi_{1}, \xi_{2}\right)\right),
\end{aligned}
$$

for all $u \in \bigwedge^{2} U^{*}$ and $\xi_{1}, \xi_{2}, \xi_{3}, \xi_{4} \in U$. We easily verify that

$$
\begin{aligned}
\phi(u)\left(v_{k}, w_{k}, v_{k}, w_{k}\right) & =(4-2 n) q\left(v_{k}, w_{k}\right) u\left(v_{k}, w_{k}\right), \\
\tau_{1} \phi(u) & =2 \tau(u) \cdot q,
\end{aligned}
$$

for all $u \in \bigwedge^{2} U^{*}$ and $1 \leq k \leq n$. From (8.6), we deduce that

$$
\phi\left(\bigwedge_{0}^{2} U^{*}\right) \subset S^{2}\left(\bigwedge_{0}^{2} U^{*}\right) .
$$

Let $\left\{\alpha_{1}, \ldots, \alpha_{n}, \beta_{1}, \ldots, \beta_{n}\right\}$ be the basis of $U^{*}$ dual to the basis of $U$ considered above. Then for $1 \leq k \leq n$, by (8.5) we see that $\phi(q)$ is non-zero and that

$$
u=\alpha_{k} \wedge \beta_{k}-(1 / 2 n) q
$$

is an element of $\bigwedge_{0}^{2} U^{*}$ which satisfies $\phi(u)\left(v_{k}, w_{k}, v_{k}, w_{k}\right) \neq 0$; thus $\phi\left(\bigwedge_{0}^{2} U^{*}\right)$ is an irreducible $S p(U)$-submodule of $S^{2}\left(\bigwedge_{0}^{2} U^{*}\right)$.

The restriction

$$
\tau_{1}: B(U) \rightarrow \bigwedge^{2} U^{*}
$$

of the morphism $\tau_{1}$ to $B(U)$ is an epimorphism of $S p(U)$-modules. Indeed, for $1 \leq k \leq n$, we see that $u_{k}=\left(\alpha_{k} \wedge \beta_{k}\right) \otimes\left(\alpha_{k} \wedge \beta_{k}\right)$ is an element of $B(U)$ satisfying $\tau_{1}\left(u_{k}\right)=\alpha_{k} \wedge \beta_{k}$; therefore the vector $q=\sum_{j=1}^{n} \alpha_{j} \wedge \beta_{j}$ and the element $\alpha_{k} \wedge \beta_{k}-(1 / 2 n) q$ of $\bigwedge_{0}^{2} U^{*}$ belong to the image of the mapping (8.7). 
From the decomposition (8.4) and the irreducibility of its summands, we deduce that the morphism (8.7) is surjective. Thus the sequence

$$
0 \rightarrow S^{2}\left(\bigwedge_{0}^{2} U^{*}\right) \rightarrow S^{2}\left(\bigwedge^{2} U^{*}\right) \stackrel{\tau_{1}}{\longrightarrow} \bigwedge^{2} U^{*} \rightarrow 0
$$

is exact. We denote by $\tau_{2}$ and $\tau_{0}$ the restrictions of the morphisms

$$
\text { id } \otimes \tau: \bigwedge^{2} U^{*} \otimes \bigwedge^{2} U^{*} \rightarrow \bigwedge^{2} U^{*}, \quad \text { id } \otimes \tau \otimes \text { id }: U^{*} \otimes \otimes^{2} U^{*} \otimes U^{*} \rightarrow U^{*} \otimes U^{*}
$$

of $S p(U)$-modules to $B(U)$, respectively; we verify that

$$
\tau_{1}(u)=\tau_{2}(u)=-2 \tau_{0}(u),
$$

for all $u \in B(U)$. It follows that the kernel $B^{0}(U)$ of the morphism (8.7) is an irreducible $S p(U)$-module whose dimension is equal to

$$
\operatorname{dim} B^{0}(U)=\operatorname{dim} B(U)-\operatorname{dim} \bigwedge^{2} U^{*}=\frac{1}{3} n(n+1)(2 n+3)(2 n-1)
$$

(see [2, Theorem 17.11]).

We know that $\bigwedge_{0}^{4} U^{*}$ vanishes when $n=3$ and is an irreducible $S p(U)$-module when $n \geq 4$. The one-dimensional subspace $\{\phi(q)\}$ of $S^{2}\left(\bigwedge_{0}^{2} U^{*}\right)$ generated by $\phi(q)$ is a trivial $S p(U)$-submodule. According to the decomposition (8.1) and the exactness of the sequence (8.8), we see that the sum of the dimensions of the $S p(U)$-modules $\{\phi(q)\}, \bigwedge_{0}^{2} U^{*}, \bigwedge_{0}^{4} U^{*}$ and $B^{0}(U)$ is equal to the dimension of $S^{2}\left(\bigwedge_{0}^{2} U^{*}\right)$. The $S p(U)$-modules $\{\phi(q)\}, \bigwedge_{0}^{2} U^{*}, B^{0}(U)$ are irreducible and pairwise non-isomorphic; when $n \geq 4$, the irreducible $S p(U)$-module $\bigwedge_{0}^{4} U^{*}$ is not isomorphic to any one of these modules. Thus we obtain the direct sum decomposition

$$
S^{2}\left(\bigwedge_{0}^{2} U^{*}\right)=\{\phi(q)\} \oplus \phi\left(\bigwedge_{0}^{2} U^{*}\right) \oplus \bigwedge_{0}^{4} U^{*} \oplus B^{0}(U)
$$

of $S^{2}\left(\bigwedge_{0}^{2} U^{*}\right)$ into $S p(n)$-submodules.

In this section, we henceforth suppose that $U=\mathbb{C}^{2 n}$ and that $q$ is the standard non-degenerate skew-symmetric bilinear form defined by

$$
q(z, w)=\left\langle z, z^{\prime}\right\rangle=\sum_{k=1}^{n}\left(z_{k} z_{n+k}^{\prime}-z_{n+k} z_{k}^{\prime}\right),
$$

where $z=\left(z_{1}, \ldots, z_{2 n}\right)$ and $z^{\prime}=\left(z_{1}^{\prime}, \ldots, z_{2 n}^{\prime}\right)$ are vectors of $U=\mathbb{C}^{2 n}$. We consider the $2 n \times 2 n$ matrix

$$
J_{n}=\left(\begin{array}{cc}
0 & I_{n} \\
-I_{n} & 0
\end{array}\right)
$$


where $I_{n}$ is the unit matrix of order $n$. Let $G^{\prime}$ be the group $S L(2 n, \mathbb{C})$; its subgroup $K^{\prime}=S p(n, \mathbb{C})$, which consists of all elements of $G^{\prime}$ preserving the bilinear form $q$, is the set of fixed points of the automorphism of $S L(2 n, \mathbb{C})$ sending the matrix $A$ into $J_{n} \cdot\left({ }^{t} A\right)^{-1} \cdot J_{n}^{-1}$.

For $1 \leq k \leq n$, the $l$-th exterior power $\bigwedge^{k} \mathbb{C}^{2 n}$ of $\mathbb{C}^{2 n}$ is an irreducible $G^{\prime}$-module, and we saw above that its $K^{\prime}$-submodule $\bigwedge_{0}^{k} \mathbb{C}^{2 n}$ is irreducible.

If $E$ is an $G^{\prime}$-module, we denote by $E^{K^{\prime}}$ the subspace of $E$ consisting of all the $K^{\prime}$-invariant elements of $E$. Then the multiplicity of an irreducible $K^{\prime}$-module $F$ in the decomposition of $E$ viewed as an $K^{\prime}$-module is equal to $\operatorname{dim} \operatorname{Hom}_{K^{\prime}}(F, E)$. Moreover, if $F$ is a $G^{\prime}$-module viewed as a $K^{\prime}$-module, the $K^{\prime}$-module $F$ is isomorphic to its contragredient module and so we have the equality

$$
\operatorname{dim} \operatorname{Hom}_{K^{\prime}}(F, E)=\operatorname{dim}(E \otimes F)^{K^{\prime}} .
$$

Throughout the remainder of this paper, for $1 \leq j, k \leq n$, we shall denote by $E_{j k}$ the element $\left(c_{l r}\right)$ of $M_{2 n}$ determined by $c_{l r}=\delta_{l j} \delta_{r k}$. We consider the Lie algebra $\mathfrak{g}=\mathfrak{s l}(2 n, \mathbb{C})$ of the group $G^{\prime}$ and its Cartan subalgebra $\mathfrak{h}$, which consists of all diagonal matrices of $\mathfrak{g}$. As in $\S 4$, let $\lambda_{j}$ be the linear form on $\mathfrak{h}$ which sends the diagonal matrix of $\mathfrak{h}$, with $a_{1}, \ldots, a_{2 n} \in \mathbb{C}$ as its diagonal entries, into $a_{j}$. We write $\alpha_{j}=\lambda_{j}-\lambda_{j+1}$, for $1 \leq j \leq 2 n-1$, and take $\left\{\alpha_{1}, \ldots, \alpha_{2 n-1}\right\}$ as a system of simple roots of $\mathfrak{g}$. If $\alpha$ is the root $\lambda_{j}-\lambda_{k}$, with $1 \leq j, k \leq 2 n-1$ and $j \neq k$, the root space $\mathfrak{g}_{\alpha}$ corresponding to $\alpha$ is generated by $E_{j k}$; as in $\S 4$, we also have the decomposition $(4.1)$ of $\mathfrak{g}$. The corresponding fundamental weights are

$$
\varpi_{j}=\lambda_{1}+\cdots+\lambda_{j}
$$

with $1 \leq j \leq 2 n-1$; we remark that $\varpi_{j}$ is the highest weight of the irreducible $G^{\prime}$-module $\bigwedge^{j} \mathbb{C}^{n}$.

The highest weight of an irreducible $G^{\prime}$-module is a linear form

$$
\varpi=a_{1} \varpi_{1}+\cdots+a_{n-1} \varpi_{n-1}
$$

on $\mathfrak{h}$, where $a_{1}, \ldots, a_{n-1} \geq 0$ are integers. The equivalence class of such an $G^{\prime}-$ module is determined by this weight. We identify the dual $\Gamma^{\prime}$ of $G^{\prime}$ with the set of all such linear forms on $\mathfrak{h}$. 
Consider the set $\Gamma_{0}^{\prime}$ of all elements $\varpi$ of $\Gamma^{\prime}$ which can be written in the form

$$
\varpi=\sum_{j=1}^{n-1} c_{j} \varpi_{2 j},
$$

where $c_{1}, \ldots, c_{n-1}$ are integers. In fact, the linear form (8.11) belongs to $\Gamma_{0}^{\prime}$ if and only if $a_{2 n-1}=0$ and $a_{2 j-1}=a_{2 j}$, for $1 \leq j \leq n-1$.

We consider an irreducible $G^{\prime}$-module $E(\varpi)$ corresponding to $\varpi \in \Gamma^{\prime}$. Let $N_{0}(\varpi)$ be the integer which is equal to 1 if $\varpi$ belongs to $\Gamma_{0}^{\prime}$ and 0 otherwise; according to a result due to Cartan (see also [6, p. 550] and Theorem 3 of [9]), we know that

$$
\operatorname{dim} E(\varpi)^{K^{\prime}}=N_{0}(\varpi)
$$

A partition $\pi=\left(\pi_{1}, \ldots, \pi_{2 n-1}\right)$ is an $(2 n-1)$-tuple of integers satisfying

$$
\pi_{1} \geq \pi_{2} \geq \cdots \geq \pi_{2 n-1} \geq 0
$$

We associate with an element $\varpi$ of $\Gamma^{\prime}$ given by (8.11) the partition

$$
\pi(\varpi)=\left(\pi_{1}, \ldots, \pi_{2 n-1}\right),
$$

where

$$
\pi_{j}=a_{1}+\cdots+a_{2 n-j},
$$

for $1 \leq j \leq n-1$; in fact, this partition uniquely determines the element $\varpi$ of $\Gamma^{\prime}$ and we shall write

$$
E(\pi(\varpi))=E(\varpi) .
$$

We denote by $P$ the set of all partitions and by $P_{0}$ its subset consisting of all partitions associated with elements of $\Gamma_{0}^{\prime}$.

Let $\varpi$ be an element of $\Gamma^{\prime}$; Pieri's formula (see [2, Proposition 15.25,(ii)]) tells us that the $G^{\prime}$-module $E(\varpi) \otimes \bigwedge^{2} \mathbb{C}^{n}$ admits a decomposition

$$
E(\varpi) \otimes \bigwedge^{2} \mathbb{C}^{n}=\bigoplus_{\eta \in \Sigma(\varpi)} E(\eta)
$$

into irreducible $G^{\prime}$-submodules, where $\Sigma(\varpi)$ is a certain subset of $P$. Each factor $E(\eta)$ appears in the sum on the right-hand side of (8.13) with multiplicity 1 . We denote by $N(\varpi)$ the cardinality of the subset $\Sigma_{0}(\varpi)=\Sigma(\varpi) \cap P_{0}$ of $\Sigma(\varpi)$. 
From the relations (8.10) and (8.12) and the decomposition (8.13), we infer that the integer

$$
\operatorname{dim} \operatorname{Hom}_{K^{\prime}}\left(\bigwedge^{2} \mathbb{C}^{n}, E(\varpi)\right)=\operatorname{dim}\left(E(\varpi) \otimes \bigwedge^{2} \mathbb{C}^{n}\right)^{K^{\prime}}
$$

is equal to $N(\varpi)$. Therefore the multiplicity $M(\varpi)$ of the irreducible $K^{\prime}$-module $\bigwedge_{0}^{2} \mathbb{C}^{n}$ in the decomposition of $E(\varpi)$ viewed as a $K^{\prime}$-module is equal to

$$
\operatorname{dim} \operatorname{Hom}_{K^{\prime}}\left(\bigwedge_{0}^{2} \mathbb{C}^{n}, E(\varpi)\right)=N(\varpi)-N_{0}(\varpi) .
$$

Thus we have

$$
M(\varpi)= \begin{cases}N(\varpi)-1 & \text { if } \varpi \text { belongs to } \Gamma_{0}^{\prime}, \\ N(\varpi) & \text { otherwise. }\end{cases}
$$

Suppose that $\varpi$ belongs to $\Gamma_{0}^{\prime}$ and that

$$
\varpi=c_{1} \varpi_{2}+c_{2} \varpi_{4}+\cdots+c_{n-1} \varpi_{2 n-2},
$$

where $c_{1}, \ldots, c_{n-1} \geq 0$ are integers, and that

$$
\pi(\varpi)=\left(\pi_{1}, \ldots, \pi_{2 n-2}, 0\right)
$$

is the partition associated with $\varpi$. For $1 \leq j \leq n-1$, consider the sequences

$$
\xi^{j}=\left(\xi_{1}^{j}, \ldots, \xi_{2 n-2}^{j}, 0\right),
$$

where $\xi_{2 j-1}^{j}=\xi_{2 j}^{j}=\pi_{2 j}+1$ and $\xi_{2 k-1}^{j}=\xi_{2 k}^{j}=\pi_{2 k}$, for $1 \leq k \leq n-1$, with $k \neq j$; we also consider the sequence

$$
\xi^{n}=\left(\pi_{1}-1, \ldots, \pi_{2 n-2}-1,0\right) .
$$

According to Pieri's formula, we easily see that $\Sigma_{0}(\varpi)$ is precisely the set of all partitions contained in $\left\{\xi^{1}, \ldots, \xi^{n}\right\}$. In fact, $\xi^{1}$ always belongs to $\Sigma_{0}(\varpi)$; moreover, for $2 \leq j \leq n-1$, the sequence $\xi^{j}$ is an element of $\Sigma_{0}(\varpi)$ if and only if $\pi_{2 j-2} \geq \pi_{2 j-1}+1$ (or equivalently if $c_{j-1} \geq 1$ ). On the other hand, the sequence $\xi^{n}$ belongs to $\Sigma_{0}(\varpi)$ if and only if $\pi_{2 n-2} \geq 1$ (or equivalently if $c_{n-1} \geq 1$ ).

We have just proved the second assertion of the following proposition; on the other hand, its first assertion is a direct consequence of the equalities (8.10) and (8.12).

Proposition 8.1. Let $G^{\prime}$ be the group $S L(2 n, \mathbb{C})$ and $K^{\prime}$ be the group $S p(n, \mathbb{C})$, with $n \geq 3$. Let $\varpi$ be an element of $\Gamma^{\prime}$. The multiplicity of the trivial $K^{\prime}$-module 
in the decomposition of the $G^{\prime}$-module $E(\varpi)$, viewed as a $K^{\prime}$-module, is equal to 1 if $\varpi$ belongs to $\Gamma_{0}^{\prime}$ and to 0 otherwise. If $\varpi$ is equal to

$$
c_{1} \varpi_{2}+c_{2} \varpi_{4}+\cdots+c_{n-1} \varpi_{2 n-2},
$$

the multiplicity $M(\varpi)$ of the $K^{\prime}$-module $\bigwedge_{0}^{2} \mathbb{C}^{n}$ in the decomposition of the $G^{\prime}$ module $E(\varpi)$, viewed as a $K^{\prime}$-module, is equal to the number of non-zero coeffcients $c_{j}$.

\section{The SYMmetric SPACE $S U(2 n) / S p(n)$}

Let $n$ be a given integer $\geq 3$. We consider the $2 n \times 2 n$ matrix

$$
J_{n}=\left(\begin{array}{cc}
0 & I_{n} \\
-I_{n} & 0
\end{array}\right) .
$$

Let $G$ be the group $S U(2 n)$ and let $K$ be the subgroup $S p(n)$ of $G$, which is equal to the set of fixed points of the involution $s$ of $G$ sending an element $A \in G$ into $J_{n} \bar{A} J_{n}^{-1}$. Then $(G, K)$ is a Riemannian symmetric pair. In the Cartan decomposition

$$
\mathfrak{g}_{0}=\mathfrak{k}_{0} \oplus \mathfrak{p}_{0}
$$

of the Lie algebra $\mathfrak{g}_{0}$ of $G$ corresponding to this involution, we know that $\mathfrak{k}_{0}$ is the Lie algebra of $K$ and that the $K$-submodule $\mathfrak{p}_{0}$ is the space of all $2 n \times 2 n$ matrices given by

$$
\mathfrak{p}_{0}=\left\{\left(\begin{array}{cc}
Z_{1} & Z_{2} \\
\bar{Z}_{2} & -\bar{Z}_{1}
\end{array}\right) \mid Z_{1} \in \mathfrak{s u}(n), Z_{2} \in \mathfrak{s o}(n, \mathbb{C})\right\} .
$$

We identify $\mathfrak{g}_{0}$ with the tangent space of $G$ at the identity element $e_{0}=I_{2 n}$ of $G$ and consider the Riemannian metric $g^{\prime}$ on $G$ defined in $\S 2$. For $1 \leq l \leq 2 n-1$, the element

$$
C_{l}=i\left(E_{l l}-E_{l+1, l+1}\right)
$$

of $M_{2 n}$ belongs to $\mathfrak{g}_{0}$. For $1 \leq l \leq n-1$, we verify that the element

$$
C_{l}^{\prime}=C_{l}+C_{n+l}
$$

of $\mathfrak{g}_{0}$ belongs to $\mathfrak{p}_{0}$; in fact, the vectors $\left\{C_{1}^{\prime}, \ldots, C_{n-1}^{\prime}\right\}$ form a basis for a maximal abelian subspace $\mathfrak{a}_{0}$ of $\mathfrak{p}_{0}$. For $1 \leq j \leq n$, we easily see that the element

$$
\tilde{C}_{j}^{\prime}=\tilde{C}_{j}+\tilde{C}_{n+j}
$$


of $\mathfrak{p}_{0}$ is also given by

$$
\tilde{C}_{j}^{\prime}=\frac{1}{n}\left(\sum_{k=j}^{n-1}(n-k) C_{k}^{\prime}-\sum_{k=1}^{j-1} k C_{k}^{\prime}\right) .
$$

By (2.1), we have

$$
g^{\prime}\left(C_{j}^{\prime}, C_{j}^{\prime}\right)=4, \quad g^{\prime}\left(C_{l}^{\prime}, C_{l+1}^{\prime}\right)=-2, \quad g^{\prime}\left(C_{k}^{\prime}, C_{q}^{\prime}\right)=0,
$$

for all $1 \leq j, k, q \leq n-1$ and $1 \leq l \leq n-2$, with $q \geq k+2$.

Let $p \geq 3$ be a given integer and $\varphi$ be an element of $T_{G, e_{0}}^{*}$. According to the relations (2.3)-(2.6), we easily see that

$$
\tilde{\sigma}_{p}^{\prime}(\varphi)\left(C_{j_{1}}^{\prime}, C_{j_{2}}^{\prime}, \ldots, C_{j_{p-1}}^{\prime}\right)=0,
$$

for $1 \leq j_{1}, j_{2}, \ldots, j_{p-1} \leq n-1$, with $j_{1}>j_{2}+1$, and that

$$
\tilde{\sigma}_{p}^{\prime}(\varphi)\left(C_{j}^{\prime}, \ldots, C_{j}^{\prime}\right)=\varphi\left(\tilde{C}_{j}^{\prime}\right)+(-1)^{p+1} \varphi\left(\tilde{C}_{j+1}^{\prime}\right),
$$

for all $1 \leq j \leq n-1$; moreover, for all $1 \leq j \leq n-2$ and $1 \leq k \leq p-1$, we have

$$
\tilde{\sigma}_{p}^{\prime}(\varphi)\left(C_{j}^{\prime}, \ldots, C_{j}^{\prime}, C_{j+1}^{\prime}, \ldots, C_{j+1}^{\prime}\right)=(-1)^{k} \varphi\left(\tilde{C}_{j+1}^{\prime}\right),
$$

if the tangent vector $C_{j}^{\prime}$ appears $k$ times in the left-hand side of this equation.

If $B$ denotes the Killing form of the Lie algebra $\mathfrak{g}_{0}$, the restriction of $-B$ to $\mathfrak{p}_{0}$ induces a $G$-invariant Riemannian metric $g_{0}$ on the homogeneous space $X=G / K$. Endowed with this metric $g_{0}$, the manifold $X$ is an irreducible symmetric space of type $A I I$ (see $\S 2$, Chapter $\mathrm{X}$ of [8]). We identify the $K$-module $\mathfrak{p}_{0}$ with the tangent space of $X$ at the coset $x_{0}$ of the identity element $e_{0}=I_{2 n}$ of $G$.

The restriction $\hat{q}_{p}$ of the $G$-invariant polynomial $Q_{p}$ on $\mathfrak{g}_{0}$ defined in $\S 2$ to $\mathfrak{p}_{0}$ is $K$-invariant and therefore gives rise to a $G$-invariant symmetric $p$-form $\sigma_{p}$ on $X$. It is well-known that the algebra of all $K$-invariant polynomials on $\mathfrak{g}_{0}$ is generated by the polynomials $\hat{q}_{p}$, with $2 \leq p \leq n$, and that these polynomials are algebraically independent. We know that the metric $g_{0}$ is equal to the symmetric 2 -form $4 n \cdot \sigma_{2}$ and that $\sigma_{3}$ is up to a constant the unique $G$-invariant symmetric 3 -form on $X$ (see $[4, \S 2]$ and $[6$, p. 560]).

Throughout the remainder of this paper, we consider the symmetric space $X=S U(2 n) / S p(n)$, with $n \geq 3$, endowed with the Riemannian metric $g=\sigma_{2}$. We consider the line bundle $\{g\}$ generated by the section $g$ of $S^{2} T^{*}$. 
For $p \geq 3$, we consider the $G$-equivariant monomorphism

$$
\tilde{\sigma}_{p}: T^{*} \rightarrow S^{p-1} T^{*}
$$

induced by the symmetric $p$-form $\sigma_{p}$. We shall write $\sigma=\sigma_{3}$ and $\tilde{\sigma}=\tilde{\sigma}_{3}$.

We consider the vector space $U=\mathbb{C}^{2 n}$ endowed with the standard nondegenerate skew-symmetric bilinear form $q$, the standard basis

$$
\left\{v_{1}, \ldots, v_{n}, w_{1}, \ldots, w_{n}\right\}
$$

of $U$ and the objects which we associated with $U$ and $q$ in $\S 8$. The isomorphism $q^{b}: U \rightarrow U^{*}$ induces an isomorphism

$$
q^{b}: \bigwedge^{2} U \rightarrow \bigwedge^{2} U^{*}
$$

of $S p(U)$-modules. We shall denote by $\bigwedge_{0}^{2} U$ the $S p(U)$-submodule of $\bigwedge^{2} U$ which isomorphic to $\bigwedge_{0}^{2} U^{*}$ under this isomorphism. We identify the $S L(U)$-modules $\operatorname{Hom}(U, U)$ and $\mathfrak{g l}(2 n, \mathbb{C})$ and we consider the monomorphism of $S p(U)$-modules

$$
\lambda: \bigwedge^{2} U \rightarrow \operatorname{Hom}(U, U)
$$

which is the restriction of the isomorphism $q^{\mathrm{b}} \otimes \mathrm{id}: \otimes^{2} U \rightarrow \operatorname{Hom}(U, U)$. Let $u$ be an element of $\bigwedge^{2} U$; we may write

$$
u=\sum_{j, k=1}^{n}\left(a_{j k} v_{j} \wedge v_{k}+b_{j k} w_{j} \wedge w_{k}+c_{j k} v_{j} \wedge w_{k}\right),
$$

where $a_{j k}, b_{j k}, c_{j k} \in \mathbb{C}$ satisfy $a_{j k}=-a_{k j}$ and $b_{j k}=-b_{k j}$. We consider the $n \times n$ matrices $A=\left(a_{j k}\right)$ and $B=\left(b_{j k}\right)$ belonging to $\mathfrak{s o}(n, \mathbb{C})$ and the $n \times n$ matrix $C=\left(c_{j k}\right)$. Then in terms of our basis of $U$, the element $\lambda(u)$ of $\operatorname{Hom}(U, U)$ is given by the $2 n \times 2 n$ matrix

$$
\left(\begin{array}{cc}
{ }^{t} C & 2 A \\
-2 B & C
\end{array}\right)
$$

We verify that the trace of $\lambda(u)$ vanishes if and only if $C$ belongs to $\mathfrak{s l}(n, \mathbb{C})$; thus $\bigwedge_{0}^{2} U$ is equal to $\lambda^{-1}(\mathfrak{s l}(2 n, \mathbb{C}))$. Let $W$ be the maximal real subspace $\lambda^{-1}(\mathfrak{s u}(n))$ of the complex vector space $\bigwedge_{0}^{2} U$. We see that $\lambda(u)$ belongs to $\mathfrak{s u}(2 n)$ if and only if $C \in \mathfrak{s u}(n)$ and $B=-\bar{A}$. Thus $\lambda$ induces an isomorphism

$$
\lambda: W \rightarrow \mathfrak{p}_{0}
$$


of $K$-modules and the image of $\bigwedge_{0}^{2} U$ under the monomorphism $\lambda$ is equal to the complexification $\mathfrak{p}$ of $\mathfrak{p}_{0}$. We consider the isomorphisms of $K$-modules

$$
\mathfrak{p}_{0} \rightarrow W, \quad \mathfrak{p} \rightarrow \bigwedge_{0}^{2} U
$$

which are equal to the inverses of the isomorphisms induced by $\lambda$. The restriction to $\bigwedge_{0}^{2} U^{*}$ of the dual of the morphism $\mathfrak{p} \rightarrow \bigwedge^{2} U$ is an isomorphism $\mu: \bigwedge_{0}^{2} U^{*} \rightarrow \mathfrak{p}^{*}$ of $K$-modules; we then consider the isomorphism

$$
\mu^{\prime}: S^{2}\left(\bigwedge_{0}^{2} U^{*}\right) \rightarrow S^{2} T_{\mathbb{C}, x_{0}}^{*}
$$

of $K$-modules induced by $\mu$. By means of $\mu^{\prime}$, the decomposition (8.9) give us a decomposition of the fiber of the vector bundle $S^{2} T_{\mathbb{C}}^{*}$ at $x_{0}$ into irreducible $K$-submodules. The fibers at $x_{0}$ of the $G$-invariant sub-bundles $\{g\}_{\mathbb{C}}$ and $\tilde{\sigma}\left(T_{\mathbb{C}}^{*}\right)$ of $S^{2} T_{\mathbb{C}}^{*}$ are irreducible $K$-submodules of $S^{2} T_{\mathbb{C}, x_{0}}^{*}$, which are isomorphic to $\mathbb{C}$ and $\bigwedge_{0}^{2} U^{*}$, respectively; the remarks made in $\S 8$ concerning the irreducible $S p(n)$-modules appearing in the decomposition (8.9) imply that the equalities

$$
\{g\}_{\mathbb{C}, x_{0}}=\mu^{\prime}(\{\phi(q)\}), \quad \tilde{\sigma}\left(T_{\mathbb{C}}^{*}\right)_{x_{0}}=\mu^{\prime}\left(\phi\left(\bigwedge_{0}^{2} U^{*}\right)\right)
$$

hold. Since a $K$-submodule $E^{0}$ of $S^{2} T_{\mathbb{C}, x_{0}}^{*}$ gives rise to a unique $G$-invariant sub-bundle $E$ of $S^{2} T_{\mathbb{C}}^{*}$ such that $E_{x_{0}}=E^{0}$, we therefore obtain the $G$-invariant decomposition

$$
S^{2} T_{\mathbb{C}}^{*}=\{g\}_{\mathbb{C}} \oplus E_{1} \oplus E_{2} \oplus \tilde{\sigma}\left(T_{\mathbb{C}}^{*}\right)
$$

of the bundle $S^{2} T_{\mathbb{C}}^{*}$, where $E_{1}$ and $E_{2}$ are the $G$-invariant sub-bundles of $S^{2} T_{\mathbb{C}}^{*}$ satisfying $E_{1, x_{0}}=\mu^{\prime}\left(\bigwedge_{0}^{4} U^{*}\right)$ and $E_{2, x_{0}}=\mu^{\prime}\left(B^{0}(U)\right)$. When $n=3$, we know that $E_{1}=\{0\}$. We denote by $S^{k} \mathfrak{p}$ the $k$-th symmetric product of $\mathfrak{p}$; since the $K$-modules $\mathfrak{p}$ and $\mathfrak{p}^{*}$ are isomorphic, from the preceding observations and the remarks made in $\S 8$ concerning the irreducible $S p(n)$-modules appearing in the decomposition (8.9), we obtain the following result:

Lemma 9.1. We have

$$
\operatorname{dim} \operatorname{Hom}_{K}\left(\mathfrak{p}, S^{2} \mathfrak{p}\right)=\operatorname{dim} \operatorname{Hom}_{K}\left(\mathfrak{p}, S^{2} T_{\mathbb{C}, x_{0}}^{*}\right)=1 .
$$

The lattice $\Lambda$ of $\mathbb{R}^{n-1}$ generated by the basis $\left\{\pi e_{j}^{\prime}\right\}_{1 \leq j \leq n-1}$ of $\mathbb{R}^{n-1}$ contains the lattice $\Lambda^{\prime}$ of $\mathbb{R}^{n-1}$ defined in $\S 2$. We consider the mapping

$$
\kappa^{\prime}: \mathbb{R}^{n-1} \rightarrow G
$$


which sends $\theta=\left(\theta_{1}, \ldots, \theta_{n-1}\right) \in \mathbb{R}^{n-1}$ into the diagonal matrix

$$
\kappa^{\prime}(\theta)=\operatorname{diag}\left(e^{i x_{1}}, \ldots, e^{i x_{n}}, e^{i x_{1}}, \ldots, e^{i x_{n}}\right)
$$

of $G$, where $x_{1}, \ldots, x_{n}$ are given by (2.11). We recall that the image $H$ of the mapping $\iota^{\prime}: \mathbb{R}^{2 n-1} \rightarrow G$ is a maximal torus of the group $G$; the image $A$ of $\kappa^{\prime}$ is a subgroup of $H$. Clearly, the Lie algebra of $A$ is equal to $\mathfrak{a}_{0}$. We denote by $\rho: G \rightarrow X$ the natural projection. Two elements $a$ and $b$ of $G$, which belong to the image of $\kappa^{\prime}$, have the same image in $X$ under $\rho$ if and only if there exists an element $\lambda \in \Lambda$ such that $a=b \kappa^{\prime}(\lambda)$. Thus there is an injective mapping

$$
\kappa: \mathbb{R}^{n-1} / \Lambda \rightarrow X
$$

such that the diagram

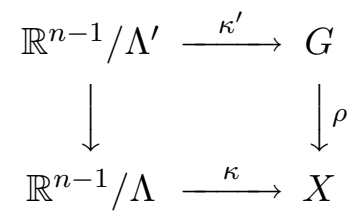

is commutative. We also denote by $\kappa$ the mapping $\rho \circ \kappa^{\prime}: \mathbb{R}^{n-1} \rightarrow X$. The subgroup $A^{\prime}=A \cap K$ of $A$ is equal to $\kappa^{\prime}(\Lambda)$ and the image $Z$ of the mappings $\kappa$ is a maximal flat totally geodesic torus of $X$, which is equal to $\rho(A)=A / A^{\prime}$. Clearly we have $\kappa(0)=\rho\left(e_{0}\right)=x_{0}$.

We consider the standard coordinate system $\left(\theta_{1}, \ldots, \theta_{n-1}\right)$ on $\mathbb{R}^{n-1}$ and endow this space with the Riemannian metric $2 \tilde{g}$, where $\tilde{g}$ is the metric on $\mathbb{R}^{n-1}$ defined in $\S 2$. For $1 \leq j \leq n-1$, we consider the vector field $\xi_{j}=\partial / \partial \theta_{j}$ on $\mathbb{R}^{n-1}$; the vector field $\zeta_{j}$ on $Z$, which is determined by

$$
\kappa_{*}\left(\xi_{j}(\theta)\right)=\zeta_{j}(\kappa(\theta)),
$$

for $\theta \in \mathbb{R}^{n-1}$, is invariant under the action of the group $A$ on $Z$. The vector field $\zeta_{j}^{\prime}+\zeta_{j+n}^{\prime}$ on $H$ is tangent to $A$; its restriction $\hat{\zeta}_{j}$ to $A$ is $\rho$-projectable and we have $\rho_{*} \hat{\zeta}_{j}=\zeta_{j}$. Since $\left\{C_{1}^{\prime}, \ldots, C_{n-1}^{\prime}\right\}$ is a frame for the submanifold $A$ of $G$, by $(9.1)$ and (9.6) we see that

$$
\kappa^{*} g=2 \tilde{g}
$$

hence the mapping $\kappa: \mathbb{R}^{n-1} / \Lambda \rightarrow Z$ is an isometric imbedding and, if $f$ is a function on $X$, we obtain the equality

$$
\int_{Z} f d Z=2^{(n-1) / 2} \sqrt{n} \int_{0}^{\pi} \cdots \int_{0}^{\pi} f(\kappa(\theta)) d \theta_{1} \ldots d \theta_{n-1},
$$


where $\theta=\left(\theta_{1}, \ldots, \theta_{n-1}\right) \in \mathbb{R}^{n-1}$. Moreover, $\left\{\zeta_{1}, \ldots, \zeta_{n-1}\right\}$ is a basis for the space of parallel vector fields on $Z$. When we identify $T_{x_{0}}$ with $\mathfrak{p}_{0}$, by $(2.12)$ we see that

$$
\zeta_{j}\left(x_{0}\right)=C_{j}^{\prime},
$$

and so $\hat{\zeta}_{j}\left(x_{0}\right)$ is equal to the vector $\zeta_{j}\left(x_{0}\right)$ of $\mathfrak{p}_{0}$ viewed as an element of $\mathfrak{g}_{0}$.

For $1 \leq j \leq n$, by (9.9) the parallel vector field

$$
\eta_{j}=\frac{1}{n}\left(\sum_{k=j}^{n-1}(n-k) \zeta_{k}-\sum_{k=1}^{j-1} k \zeta_{k}\right)
$$

on $Z$ satisfies $\eta_{j}\left(x_{0}\right)=\tilde{C}_{j}^{\prime}$; then we verify that

$$
\zeta_{j}=\eta_{j}-\eta_{j+1}
$$

for $1 \leq j \leq n-1$, and that

$$
\sum_{j=1}^{n} \eta_{j}=0
$$

Let $\varphi$ be an element of $T_{x_{0}}^{*}$; then there is a unique element $\zeta \in \mathfrak{p}_{0}$ such that $\varphi=g^{b}(\zeta)$. Since we have $g^{\prime}\left(\mathfrak{p}_{0}, \mathfrak{k}_{0}\right)=0$, the element $\rho^{*} \varphi$ of $T_{G, e_{0}}^{*}$ is equal to $g^{\prime \prime}\left(\zeta^{\prime}\right)$, where $\zeta^{\prime}$ is equal to the vector $\zeta$ viewed as an element of $\mathfrak{g}_{0}$. If $p \geq 3$ is a given integer, we therefore have the equalities

$$
\varphi\left(y_{1}\right)=\left(\rho^{*} \varphi\right)\left(y_{1}^{\prime}\right), \quad \tilde{\sigma}_{p}(\varphi)\left(y_{1}, \ldots, y_{p-1}\right)=\tilde{\sigma}_{p}^{\prime}\left(\rho^{*} \varphi\right)\left(y_{1}^{\prime}, \ldots, y_{p-1}^{\prime}\right),
$$

for all $y_{1}, \ldots, y_{p-1} \in \mathfrak{p}_{0}$, where $y_{j}^{\prime}$ is equal to the vector $y_{j}$ considered as an element of $\mathfrak{g}_{0}$. Then from the equalities (9.2)-(9.4), we obtain the relation

$$
\tilde{\sigma}_{p}(\varphi)\left(\zeta_{j_{1}}, \zeta_{j_{2}}, \ldots, \zeta_{j_{p-1}}\right)=0
$$

for $1 \leq j_{1}, j_{2}, \ldots, j_{p-1} \leq n-1$, with $j_{1}>j_{2}+1$, and the relations

$$
\tilde{\sigma}_{p}(\varphi)\left(\zeta_{j}, \ldots, \zeta_{j}\right)=\varphi\left(\eta_{j}\right)+(-1)^{p+1} \varphi\left(\eta_{j+1}\right),
$$

for all $1 \leq j \leq n-1$; moreover, for all $1 \leq j \leq n-2$ and $1 \leq k \leq p-1$, we have

$$
\tilde{\sigma}_{p}(\varphi)\left(\zeta_{j}, \ldots, \zeta_{j}, \zeta_{j+1}, \ldots, \zeta_{j+1}\right)=(-1)^{k} \varphi\left(\eta_{j+1}\right),
$$

if the vector field $\zeta_{j}$ appears $k$ times in the left-hand side of this equation. Because the vector fields $\zeta_{j}$ are invariant under the action of the group $A$, the relations (9.12)-(9.14) hold for all $\varphi \in C^{\infty}\left(T^{*}\right)$. Since $\left\{\zeta_{1}, \ldots, \zeta_{n-1}\right\}$ is a basis for the space of parallel vector fields on $Z$, and since all maximal flat totally geodesic 
tori of $X$ are conjugate under the action of $G$ on $X$, from the relations (9.10) and (9.12)-(9.14) we deduce the following result:

Lemma 9.2. Let $n, p \geq 3$ be given integers and let $X$ be the symmetric space $S U(2 n) / S p(n)$. A 1-form $\varphi$ on $X$ satisfies the Guillemin condition if and only if the symmetric $(p-1)$-form $\tilde{\sigma}_{p}(\varphi)$ on $X$ satisfies the Guillemin condition.

According to (9.11), (9.13) and (9.14), we easily see that an arbitrary element $h$ of the sub-bundle $\tilde{\sigma}\left(T^{*}\right)$ of $S^{2} T^{*}$ satisfies the relation

$$
\sum_{j=1}^{n-1} h\left(\zeta_{j}, \zeta_{j}\right)+\sum_{j=1}^{n-2} h\left(\zeta_{j}, \zeta_{j+1}\right)=0 .
$$

If $\varphi$ is a 1 -form on $X$, since the mapping $\kappa$ is totally geodesic, by (9.6) and the definition of the operator $D^{1}$ we have the equality

$$
2 \kappa^{*}\left(D^{1} \varphi\right)\left(\zeta_{j}, \zeta_{k}\right)=\xi_{j} \cdot\left\langle\xi_{k}, \kappa^{*} \varphi\right\rangle+\xi_{k} \cdot\left\langle\xi_{j}, \kappa^{*} \varphi\right\rangle
$$

of functions on $\mathbb{R}^{n-1}$, for $1 \leq j, k \leq n-1$.

\section{FunCtions on the Symmetric SPACE $S U(2 n) / S p(n)$}

We consider the group $G=S U(2 n)$, with $n \geq 3$, and its Lie algebra $\mathfrak{g}_{0}$. We also consider the Lie algebra $\mathfrak{h}_{0}$ of the maximal torus $H$ of $G$; the complexification $\mathfrak{h}$ of $\mathfrak{h}_{0}$ is equal to the Cartan subalgebra of the complexification $\mathfrak{g}$ of $\mathfrak{g}_{0}$ consisting of all diagonal matrices. We shall consider the objects associated with $\mathfrak{g}$ in $\S 4$ or $\S 8$, namely the decomposition (4.1) of $\mathfrak{g}$ and the fundamental weights $\varpi_{j}$, with $1 \leq j \leq 2 n-1$. We identify the dual $\Gamma$ of $G$ with the set of all linear forms

$$
\gamma=\gamma_{r_{1}, \ldots, r_{2 n-1}}=r_{1} \varpi_{1}+\cdots+r_{2 n-1} \varpi_{2 n-1},
$$

where $r_{1}, \ldots, r_{2 n-1}$ are non-negative integers.

We consider the $G$-module structure on $C^{\infty}(G)$ arising from the left action of $G$ on itself, the corresponding left regular representation $\pi$ of $G$ on $C^{\infty}(G)$ and the isotypic component $C_{\gamma}^{\infty}(G)$ of the $G$-module $C^{\infty}(G)$ corresponding to $\gamma \in \Gamma$.

As in $\S 3$, we consider the group $G$ as a real submanifold of the complex manifold $M_{2 n}$. We shall consider the left and right action of the group $G$ on the manifold $M_{2 n}$. The left action of $G$ on $M_{2 n}$ induces a morphism $\Phi$ from $\mathfrak{g}$ to the 
Lie algebra of complex vector fields on $M_{2 n}$, which are tangent to the submanifold $G$ of $M_{2 n}$.

For $1 \leq j, k \leq 2 n$, we denote by $z_{j k}$ the function on the space of matrices $M_{2 n}$ which sends a matrix of $M_{2 n}$ into its $(j, k)$-th entry. For $1 \leq j \leq 2 n$, we consider the $\mathbb{C}^{2 n}$-valued functions $Z_{j}$ on $M_{2 n}$ which sends a matrix of $M_{2 n}$ into its $j$-th row; then we have $Z_{j}=\left(z_{j 1}, \ldots, z_{j, 2 n}\right)$. For $1 \leq j, k \leq 2 n$, the complex vector field $\xi_{j k}$ on $M_{2 n}$ defined in $\S 3$ satisfies

$$
\xi_{j k} Z_{l}=\delta_{k l} Z_{j},
$$

for all $1 \leq l \leq 2 n$.

We consider the group $S p(n, \mathbb{C})$ of automorphisms of $\mathbb{C}^{2 n}$ which preserve the standard non-degenerate skew-symmetric bilinear form $q=\langle$,$\rangle on \mathbb{C}^{2 n}$. The subgroup $K=S p(n)$ of $G$ is equal to $S U(2 n) \cap S p(n, \mathbb{C})$, and it is easily seen that the transpose of an element of $K$ belongs to $S p(n, \mathbb{C})$; it follows that, for $1 \leq j, k \leq 2 n$, the complex-valued function $\left\langle Z_{j}, Z_{k}\right\rangle$ on $M_{2 n}$ is invariant under the right action of $K$ on the space $M_{2 n}$. Let $1 \leq k \leq n-1$ be a given integer; the function $A_{k}$ on $M_{2 n}$ defined by

$$
A_{k}=\left(\left\langle Z_{j}, Z_{l}\right\rangle\right)_{1 \leq j, l \leq 2 k}
$$

takes its values in the space of skew-symmetric matrices of $M_{2 k}$. Clearly the complex-valued function

$$
f_{k}=\operatorname{Pfaff}\left(A_{k}\right)
$$

on $M_{2 n}$, which assigns to a point $x$ of $M_{2 n}$ the Pfaffian of the skew-symmetric matrix $A_{k}(x)$, is also invariant under the right action of $K$. According to formula (8.2), the function $f_{k}$ is given by

$$
f_{k}=\frac{1}{k ! 2^{k}} \sum_{\tau \in \mathfrak{S}_{2 k}} \operatorname{sgn} \tau \cdot \prod_{j=1}^{k}\left\langle Z_{\tau(2 j-1)}, Z_{\tau(2 j)}\right\rangle=\frac{1}{k !} q^{k}\left(Z_{1}, \ldots, Z_{2 k}\right) ;
$$

in particular, we have $f_{1}=\left\langle Z_{1}, Z_{2}\right\rangle$ and

$$
f_{2}=\left\langle Z_{1}, Z_{2}\right\rangle\left\langle Z_{3}, Z_{4}\right\rangle-\left\langle Z_{1}, Z_{3}\right\rangle\left\langle Z_{2}, Z_{4}\right\rangle+\left\langle Z_{1}, Z_{4}\right\rangle\left\langle Z_{2}, Z_{3}\right\rangle
$$

(see [6, pp. 627-628]). We consider the element

$$
\phi=\sum_{j=1}^{n-1}\left(E_{2 j-1, j}+E_{2 j, n+j}\right)+E_{2 n-1, n}+(-1)^{n} E_{2 n, 2 n}
$$


of $S O(2 n)$; then if $B$ is an element of $M_{2 n}$ whose $j$-th row is the vector $B_{j}$ of $\mathbb{C}^{2 n}$, the $k$-th row $B_{k}^{\prime}$ of the matrix $B^{\prime}=\phi \cdot B$ is given by

$$
B_{2 j-1}^{\prime}=B_{j}, \quad B_{2 j}^{\prime}=B_{n+j},
$$

for $1 \leq j \leq n-1$. Hence if $\left\{\varepsilon_{1}, \ldots, \varepsilon_{2 n}\right\}$ is the standard basis of $\mathbb{C}^{2 n}$ and $\theta=\left(\theta_{1}, \ldots, \theta_{n-1}\right) \in \mathbb{R}^{n-1}$, we have

$$
Z_{2 j-1}\left(\phi \kappa^{\prime}(\theta)\right)=e^{i x_{j}} \varepsilon_{j}, \quad Z_{2 j}\left(\phi \kappa^{\prime}(\theta)\right)=e^{i x_{j}} \varepsilon_{n+j},
$$

for $1 \leq j \leq n-1$, where $x_{1}, \ldots, x_{n}$ are given by (2.13). For $1 \leq k \leq n-1$, the function $f_{k}^{\prime}=\phi^{*} f_{k}$ on $M_{2 n}$ is also invariant under the right action of $K$; from the previous relations, it follows that

$$
f_{k}^{\prime}\left(\kappa^{\prime}(\theta)\right)=e^{2 i \theta_{k}}
$$

Lemma 10.1. For $1 \leq k \leq n-1$ and $1 \leq j, l \leq 2 n$ be given integers. The equalities

$$
\xi_{j l} f_{k}=0
$$

hold on $M_{2 n}$ whenever $l>2 k$, and the equalities

$$
\xi_{j l} f_{k}=\delta_{j l} f_{k}
$$

hold on $M_{2 n}$ whenever $1 \leq j, l \leq 2 k$.

Proof. The first equalities are immediate. Suppose that $1 \leq j, l \leq 2 k$ and let $W_{1}, \ldots, W_{2 k}$ be the elements of $\mathbb{C}^{2 n}$ defined by

$$
W_{r}=\delta_{r l} Z_{j}+\left(1-\delta_{r l}\right) Z_{r},
$$

for $1 \leq r \leq 2 k$. Since $q^{k}$ is an element of $\bigwedge^{2 k} U^{*}$, according to (10.1) and (10.2) we see that

$$
k ! \xi_{j l} f_{k}=q^{k}\left(W_{1}, \ldots, W_{2 k}\right)=\delta_{j l} q^{k}\left(Z_{1}, \ldots, Z_{2 k}\right)=k ! \delta_{j l} f_{k} .
$$

Let $1 \leq k \leq n-1$ and $1 \leq j<l \leq 2 n$ be given integers; the preceding lemma implies that the equalities

$$
\Phi\left(C_{l}\right) f_{k}=-\varpi_{2 k}\left(C_{l}\right) f_{k}, \quad \Phi\left(E_{l j}\right) f_{k}=-\xi_{j l} f_{k}=0,
$$

hold on $M_{2 n}$. 
Throughout the remainder of this paper, by $f_{k}$ we shall always often mean the restriction of the function $f_{k}$ on $M_{2 n}$ to the submanifold $G$. From the relations (10.4) and (3.3), it follows that

$$
\pi(\xi) \bar{f}_{k}=\varpi_{2 k}(\xi) \bar{f}_{k}, \quad \pi(\eta) \bar{f}_{k}=0,
$$

for all $\xi \in \mathfrak{h}_{0}$ and $\eta \in \mathfrak{n}^{+}$. Thus the function $\bar{f}_{k}$ is a highest weight vector of the isotypic component $C_{\varpi_{2 k}}^{\infty}(G)$; moreover according to (4.3) and (4.6), we know that $f_{k}$ is an element of $C_{\varpi_{2(n-k)}}^{\infty}(G)$.

Let $r_{1}, \ldots, r_{n-1} \geq 0$ be given integers and $\gamma$ be the element

$$
\gamma_{r_{1}, \ldots, r_{n-1}}^{\prime}=\sum_{k=1}^{n-1} r_{k} \varpi_{2 k}
$$

of $\Gamma$; we consider the complex-valued function $f_{r_{1}, \ldots, r_{n-1}}$ on $G$ defined by

$$
f_{r_{1}, \ldots, r_{n-1}}=\prod_{k=1}^{n-1} f_{k}^{r_{k}}
$$

Since $\bar{f}_{k}$ is a highest weight vector of the $G$-module $C_{\varpi_{2 k}}^{\infty}(G)$, we know that $\bar{f}_{r_{1}, \ldots, r_{n-1}}$ is a highest weight vector of the $G$-module $C_{\gamma}^{\infty}(G)$.

If $\gamma$ is an element of $\Gamma$, we consider an irreducible $G$-module $E_{\gamma}$ corresponding to $\gamma$. We shall denote by $\Gamma_{0}$ the subset of $\Gamma$ consisting of all elements $\gamma_{r_{1}, \ldots, r_{n-1}}^{\prime}$ of $\Gamma$, where $r_{1}, \ldots, r_{n-1} \geq 0$ are integers. Since the group $G$ is a real form of the group $S L(2 n, \mathbb{C})$ and the subgroup $K$ is equal to $G \cap S p(n, \mathbb{C})$, from Proposition 8.1 we deduce the following result:

Proposition 10.2. Let $G$ be the group $S U(2 n)$ and $K$ be its subgroup $S p(n)$, with $n \geq 3$. The multiplicity of the trivial $K$-module in the decomposition of the $G$-module $E_{\gamma}$, viewed as a $K$-module, is equal to 1 if $\gamma$ belongs to $\Gamma_{0}$ and to 0 otherwise. If $r_{1}, \ldots, r_{n-1}$ are given integers $\geq 0$ and $\gamma$ is the element $\gamma_{r_{1}, \ldots, r_{n-1}}^{\prime}$ of $\Gamma_{0}$, the multiplicity of the $K$-module $\mathfrak{p}$ in the decomposition of the $G$-module $E_{\gamma}$, viewed as a $K$-module, is equal to the number of non-zero integers $r_{j}$.

We now consider the symmetric space $X=G / K$. For $\gamma \in \Gamma$, we recall that the multiplicity of the $G$-module $C_{\gamma}^{\infty}\left(S^{p} T_{\mathbb{C}}^{*}\right)$ is equal to the dimension of the weight subspace of the $G$-module $C_{\gamma}^{\infty}\left(S^{p} T_{\mathbb{C}}^{*}\right)$ corresponding to $\gamma$ (see $\S 2$, Chapter II of [3]). A linear form $\lambda$ on $\mathfrak{h}$ is a weight of the $G$-module $C_{\gamma}^{\infty}\left(S^{p} T_{\mathbb{C}}^{*}\right)$ if and only 
if $-\lambda$ is a weight of the complex conjugate $\overline{C_{\gamma}^{\infty}\left(S^{p} T_{\mathbb{C}}^{*}\right)}$ of the space $C_{\gamma}^{\infty}\left(S^{p} T_{\mathbb{C}}^{*}\right)$; therefore we have the equality

$$
C_{\bar{\gamma}}^{\infty}\left(S^{p} T_{\mathbb{C}}^{*}\right)=\overline{C_{\gamma}^{\infty}\left(S^{p} T_{\mathbb{C}}^{*}\right)}
$$

of $G$-modules.

If $E$ is a $G$-submodule of $C^{\infty}(G)$, we denote by $E^{K}$ the $G$-submodule of $E$ consisting of all functions of $E$ which are invariant under the right action of $K$ on $G$. The natural projection $\rho: G \rightarrow X$ induces an isomorphism

$$
\rho^{*}: C^{\infty}(X) \rightarrow C^{\infty}(G)^{K}
$$

of $G$-modules, which sends a function $f \in C^{\infty}(X)$ into the function $\rho^{*} f$ on $G$. If $\gamma$ is an element of $\Gamma$, this mapping $\rho^{*}$ induces a monomorphism

$$
\rho^{*}: C_{\gamma}^{\infty}(X) \rightarrow C_{\gamma}^{\infty}(G)^{K} .
$$

A function $f$ on $G$ which is invariant under the right action of $K$ on $G$ determines a function $\tilde{f}$ on $X$ satisfying $\rho^{*} \tilde{f}=f$.

For $1 \leq k \leq n-1$, since the function $f_{k}$ on $M_{n}$ is invariant under the right action of $K$ on $M_{n}$, its restriction to $G$ induces by passage to the quotient a function $\tilde{f}_{k}$ on $X$. The complex conjugate $\hat{f}_{k}$ of the function $\tilde{f}_{k}$ is equal to the function on $X$ induced by the function $\bar{f}_{k}$ on $G$. The restriction of the function $f_{k}^{\prime}$ to $G$ induces a function $\tilde{f}_{k}^{\prime}$ on $X$ which is equal to $\phi^{*} \tilde{f}_{k}$. If $r_{1}, \ldots, r_{n-1} \geq 0$ are integers, the function

$$
\tilde{f}_{r_{1}, \ldots, r_{n-1}}=\prod_{k=1}^{n-1} \tilde{f}_{k}^{r_{k}}
$$

is equal to the function on $X$ induced by the function $f_{r_{1}, \ldots, r_{n-1}}$ on $G$; its complex conjugate $\hat{f}_{r_{1}, \ldots, r_{n-1}}$ is equal to the function on $X$ induced by the function $\bar{f}_{r_{1}, \ldots, r_{n-1}}$ on $G$. If $r_{1}, \ldots, r_{n-1} \in \mathbb{Z}$, when at least one of the integers is $<0$, we set

$$
\tilde{f}_{r_{1}, \ldots, r_{n-1}}=0 .
$$

For $r_{1}, \ldots, r_{n-1} \in \mathbb{Z}$, we consider the function $\tilde{f}_{r_{1}, \ldots, r_{n-1}}^{\prime}=\phi^{*} \tilde{f}_{r_{1}, \ldots, r_{n-1}}$.

If $\gamma$ is an element of $\Gamma$, by the Frobenius reciprocity theorem the first assertion of Proposition 10.2 tells us that the isotypic component $C_{\gamma}^{\infty}(X)$ of $C^{\infty}(X)$ corresponding to $\gamma$ is irreducible if $\gamma$ belongs to $\Gamma_{0}$ and vanishes whenever $\gamma$ does not belong to $\Gamma_{0}$. 
If $r_{1}, \ldots, r_{n-1} \geq 0$ are given integers and $\gamma$ is the element $\gamma_{r_{1}, \ldots, r_{n-1}}^{\prime}$ of $\Gamma_{0}$, since $\bar{f}_{r_{1}, \ldots, r_{n-1}}$ is a highest weight vector of the $G$-module $C_{\gamma}^{\infty}(G)$, the function $\hat{f}_{r_{1}, \ldots, r_{n-1}}$ is a highest weight vector of the irreducible $G$-module $C_{\gamma}^{\infty}(X)$. In particular, for $1 \leq k \leq n-1$, we know that $\hat{f}_{k}$ is a highest weight vector of the irreducible $G$-module $C_{\varpi_{2 k}}^{\infty}(X)$.

If $\gamma \in \Gamma_{0}$, according to (4.6) or (10.6) we have the equality

$$
C_{\bar{\gamma}}^{\infty}(X)=\overline{C_{\gamma}^{\infty}(X)}
$$

of $G$-modules. Hence by (4.3), we see that $\tilde{f}_{k}$ is an element of $C_{\varpi_{2(n-k)}}^{\infty}(X)$, for $1 \leq k \leq n-1$. The element $\gamma=\gamma_{1,0, \ldots, 0,1}^{\prime}=\varpi_{2}+\varpi_{2 n-2}$ of $\Gamma_{0}$ satisfies $\bar{\gamma}=\gamma$; thus according to (10.7), the $G$-module $\mathcal{B}=C_{\gamma}^{\infty}(X)$ is invariant under complex conjugation, and hence is equal to the complexification of the $G$-submodule

$$
\mathcal{B}_{\mathbb{R}}=\{f \in \mathcal{B} \mid f=\bar{f}\}
$$

of $C_{\mathbb{R}}^{\infty}(X)$. Since the function $\hat{f}_{1} \cdot \hat{f}_{n-1}$ is a highest weight vector of $\mathcal{B}$, its complex conjugate $\tilde{f}_{1} \cdot \tilde{f}_{n-1}$ is also an element of $\mathcal{B}$.

Let $r_{1}, \ldots, r_{n-1} \geq 0$ be given integers and $\gamma$ be the element $\gamma_{r_{1}, \ldots, r_{n-1}}^{\prime}$ of $\Gamma_{0}$; by the Frobenius reciprocity theorem and the second assertion of Proposition 10.2, we see that the multiplicity of the isotypic component $C_{\gamma}^{\infty}\left(T_{\mathbb{C}}^{*}\right)$ of $C^{\infty}\left(T_{\mathbb{C}}^{*}\right)$ is equal to the number of non-zero integers $r_{1}, \ldots, r_{n-1}$. Since $\hat{f}_{k}$ is not a constant function, we know that $d \hat{f}_{k}$ is a highest weight vector of the $G$-module $C_{\varpi_{2 k}}^{\infty}\left(T_{\mathbb{C}}^{*}\right)$. Therefore the section $\hat{f}_{r_{1}, \ldots, r_{n-1}} d \hat{f}_{k}$ is a highest weight vector of the $G$-module $C_{\gamma^{\prime}}^{\infty}\left(T_{\mathbb{C}}^{*}\right)$, where

$$
\gamma^{\prime}=\gamma_{r_{1}, \ldots, r_{k-1}, r_{k}+1, r_{k+1}, \ldots, r_{n-1}}^{\prime}=\varpi_{2 k}+\gamma_{r_{1}, \ldots, r_{n-1}}^{\prime} .
$$

We consider the sections $\varphi_{1}, \ldots, \varphi_{n-1}$ of $T_{\mathbb{C}}^{*}$ defined by

$$
\varphi_{k}=\tilde{f}_{r_{1}, \ldots, r_{k-1}, r_{k}-1, r_{k+1}, \ldots, r_{n-1}} d \tilde{f}_{k},
$$

for $1 \leq k \leq n-1$. Note that $\varphi_{k}$ is non-zero if and only if the integer $r_{k}$ is non-zero. If $\varphi_{k}$ is non-zero, we have just seen that the complex conjugate $\bar{\varphi}_{k}$ of $\varphi_{k}$ is a highest weight vector of the $G$-module $C_{\gamma}^{\infty}\left(T_{\mathbb{C}}^{*}\right)$. In the next section, we shall verify that the non-zero elements of the family $\left\{\varphi_{1}, \ldots, \varphi_{n-1}\right\}$ are linearly independent. We know that the number of such elements of this family is equal to the number of non-zero integers $r_{k}$. On the other hand, we remarked above that the latter number is equal to the multiplicity of the isotypic component 
$C_{\gamma}^{\infty}\left(T_{\mathbb{C}}^{*}\right)$ and hence also to the dimension of the weight subspace $\mathcal{W}_{\gamma}$ of $C_{\gamma}^{\infty}\left(T_{\mathbb{C}}^{*}\right)$ corresponding to its highest weight $\gamma$. Therefore we have the following result:

Lemma 10.3. Let $r_{1}, \ldots, r_{n-1} \geq 0$ be given integers and let $\gamma$ be the element $\gamma_{r_{1}, \ldots, r_{n-1}}^{\prime}$ of $\Gamma$. Then the non-zero members of the family $\left\{\bar{\varphi}_{1}, \ldots, \bar{\varphi}_{n-1}\right\}$ associated with the integers $r_{1}, \ldots, r_{n-1}$ form a basis for the space $\mathcal{W}_{\gamma}$.

By Lemma 10.3, we know that the $G$-module $C_{\varpi_{2}}^{\infty}\left(T_{\mathbb{C}}^{*}\right)$ is irreducible and we have the equality

$$
C_{\varpi_{2}}^{\infty}\left(T_{\mathbb{C}}^{*}\right)=d C_{\varpi_{2}}^{\infty}(X)
$$

The space $U=\mathbb{C}^{2 n}$ is a $G$-module, and so the $k$-th exterior products $\bigwedge^{2} U$ and $\bigwedge^{2} U^{*}$ inherit structures of $G$-modules. In fact, the highest weight of the irreducible $G$-module $\bigwedge^{2} U$ is $\varpi_{2}$ and this $G$-module viewed as a $K$-module is isomorphic to $\bigwedge^{2} U^{*}$. If $q$ is the standard non-degenerate skew-symmetric bilinear form on $U$, we know that (8.4) is the decomposition of the $G$-module $\wedge^{2} U^{*}$ into irreducible $K$-modules; according to remarks made in $\S \S 8$ and 9 , we then see that

$$
\operatorname{Hom}_{K}\left(E_{1, x_{0}, \mathbb{C}}, \bigwedge^{2} U\right)=\operatorname{Hom}_{K}\left(E_{2, x_{0}, \mathbb{C}}, \bigwedge^{2} U\right)=\{0\} .
$$

Therefore from the Frobenius reciprocity theorem, we obtain the following result:

Lemma 10.4. We have

$$
C_{\varpi_{2}}^{\infty}\left(E_{1, \mathbb{C}}\right)=C_{\varpi_{2}}^{\infty}\left(E_{2, \mathbb{C}}\right)=\{0\} .
$$

From Lemma 10.4, the equality (10.8) and the decomposition (9.5), we obtain the decomposition

$$
C_{\varpi_{2}}^{\infty}\left(S^{2} T_{\mathbb{C}}^{*}\right)=C_{\varpi_{2}}^{\infty}(X) \cdot g \oplus \tilde{\sigma} d C_{\varpi_{2}}^{\infty}(X) .
$$

Thus the multiplicity of the isotypic component $C_{\varpi_{2}}^{\infty}\left(S^{2} T_{\mathbb{C}}^{*}\right)$ is equal to 2 and the weight subspace of this $G$-module corresponding to its highest weight $\varpi_{2}$ is generated by the sections $\hat{f}_{1} g$ and $\tilde{\sigma}\left(d \hat{f}_{1}\right)$.

\section{Isospectral DEFormations of $S U(2 n) / S p(n)$}

We pursue our study of the symmetric space $X=S U(2 n) / S p(n)$, with $n \geq 3$. This section is devoted to the proof of the following proposition: 
Proposition 11.1. We have

$$
D_{0} C^{\infty}(T) \cap \tilde{\sigma} d C_{\mathbb{R}}^{\infty}(X)=\tilde{\sigma} d \mathcal{B}_{\mathbb{R}}, \quad D_{0} C^{\infty}\left(T_{\mathbb{C}}\right) \cap \tilde{\sigma} d C^{\infty}(X)=\tilde{\sigma} d \mathcal{B} .
$$

We consider the orthogonal complement $\mathcal{F}$ of the finite-dimensional subspace $\mathcal{F}^{\prime}=\mathbb{R}(X) \oplus \mathcal{B}_{\mathbb{R}}$ of $C_{\mathbb{R}}^{\infty}(X)$. According to Lemma 9.2, we know that the mapping

$$
P_{\sigma}=P \tilde{\sigma} d: C_{\mathbb{R}}^{\infty}(X) \rightarrow I(X)
$$

is well-defined. Proposition 11.1 tells us that the kernel of $P_{\sigma}$ is the finitedimensional space $\mathcal{F}^{\prime}$ and that the mapping $P_{\sigma}: \mathcal{F} \rightarrow I(X)$ is injective.

Let $r_{1}, \ldots, r_{n-1} \geq 0$ be given integers which are not all zero. We consider the element $\gamma=\gamma_{r_{1}, \ldots, r_{n-1}}^{\prime}$ of $\Gamma_{0}$ and the subspace $\mathcal{V}_{\gamma}$ of $C^{\infty}\left(T_{\mathbb{C}}^{*}\right)$ generated by the 1 -forms $\left\{\varphi_{1}, \ldots, \varphi_{n-1}\right\}$, which we associated in $\S 10$ with the integers $r_{1}, \ldots, r_{n-1}$. According to Lemma 10.3, the complex conjugate of the space $\mathcal{V}_{\gamma}$ is equal to the highest weight subspace $\mathcal{W}_{\gamma}$ of $C_{\gamma}^{\infty}\left(T_{\mathbb{C}}^{*}\right)$. We consider the section

$$
\varphi=\sum_{k=1}^{n-1} a_{k} \varphi_{k}
$$

of $T_{\mathbb{C}}^{*}$, where $a_{1}, \ldots, a_{n-1}$ are given complex numbers, and the 1 -form

$$
\vartheta=d \tilde{f}_{r_{1}, \ldots, r_{n-1}}=\sum_{k=1}^{n-1} r_{k} \varphi_{k}
$$

on $X$, which is also an element of $\mathcal{V}_{\gamma}$. We also consider the 1 -forms $\varphi^{\prime}=\phi^{*} \varphi$ and $\vartheta^{\prime}=\phi^{*} \vartheta$ on $X$. For our proof of Proposition 11.1, we shall require the following result:

Lemma 11.2. Let $r_{1}, \ldots, r_{n-1} \geq 0$ be given integers which are not all zero, and let $a_{1}, \ldots, a_{n-1}$ be given complex numbers. Suppose that there is an element $c \in \mathbb{C}$ such that the 1-form

$$
\varphi=\sum_{k=1}^{n-1} a_{k} \varphi_{k}
$$

satisfies the relation

$$
D^{1} \varphi=c \tilde{\sigma}\left(d \tilde{f}_{r_{1}, \ldots, r_{n-1}}\right) .
$$

Then either the coefficient $c$ vanishes or we have

$$
r_{k}=0, \quad r_{1}=r_{n-2}=1, \quad \varphi=a\left(\tilde{f}_{n-1} d \tilde{f}_{1}-\tilde{f}_{1} d \tilde{f}_{n-1}\right), \quad c=-2 i a,
$$

for $2 \leq k \leq n-2$, where $a=a_{1}=-a_{n-1}$. 
We assume, without any loss of generality, that $a_{k}=0$ whenever $r_{k}=0$, for $1 \leq k \leq n-1$. We consider the function $\psi$ on $\mathbb{R}^{n-1}$ defined by

$$
\psi(\theta)=2 i e^{2 i\left(r_{1} \theta_{1}+\cdots+r_{n-1} \theta_{n-1}\right)},
$$

for $\theta=\left(\theta_{1}, \ldots, \theta_{n-1}\right) \in \mathbb{R}^{n-1}$; then we have

$$
\xi_{k} \cdot \psi=2 i r_{k} \psi
$$

for $1 \leq k \leq n-1$. By (10.3), we see that

$$
\kappa^{*} \tilde{f}_{r_{1}, \ldots, r_{n-1}}^{\prime}=\frac{1}{2 i} \psi, \quad\left\langle\xi_{j}, \kappa^{*} d \tilde{f}_{k}^{\prime}\right\rangle=2 i \delta_{j k} \kappa^{*} \tilde{f}_{k}^{\prime},
$$

for $1 \leq j, k \leq n-1$. Thus we have

$$
\left\langle\xi_{k}, \kappa^{*} \varphi^{\prime}\right\rangle=a_{k} \psi
$$

for $1 \leq k \leq n-1$. By formulas (9.16), (11.2) and (11.3), we obtain

$$
\kappa^{*}\left(D^{1} \varphi^{\prime}\right)\left(\zeta_{k}, \zeta_{l}\right)=i\left(a_{k} r_{l}+a_{l} r_{k}\right) \psi,
$$

for $1 \leq k, l \leq n-1$.

If the 1 -form $\varphi$ vanishes, then according to formula (11.4), with $k=l$, we see that $a_{k} r_{k}=0$, for all $1 \leq k \leq n-1$; hence in this case all the coefficients $a_{k}$ vanish. In other words, the non-zero members of the family $\left\{\varphi_{1}, \ldots, \varphi_{n-1}\right\}$ are linearly independent. This fact entered into the proof of Lemma 10.3.

According to (9.6) and (11.3), we have

$$
\kappa^{*} \vartheta^{\prime}\left(\zeta_{k}\right)=\left\langle\xi_{k}, \kappa^{*} \vartheta^{\prime}\right\rangle=r_{k} \psi
$$

for $1 \leq k \leq n-1$; thus we obtain

$$
\kappa^{*} \vartheta^{\prime}\left(\eta_{j}\right)=d_{j} \psi
$$

for $1 \leq j \leq n$, where the number $d_{j}$ is given by formula (8.1) of [5]. We also consider the symmetric 2 -form $h=\tilde{\sigma}\left(\vartheta^{\prime}\right)$. By (9.12), we have

$$
h\left(\zeta_{j}, \zeta_{k}\right)=0,
$$

for $1 \leq j, k \leq n-1$, whenever $k \geq j+2$. Also by (9.13) and (9.14), we see that

$$
h\left(\zeta_{j}, \zeta_{j}\right)=\vartheta^{\prime}\left(\eta_{j}+\eta_{j+1}\right), \quad h\left(\zeta_{k}, \zeta_{k+1}\right)=-\vartheta^{\prime}\left(\eta_{k+1}\right),
$$

for all $1 \leq j \leq n-1$ and $1 \leq k \leq n-2$. 
We now begin the proof of Lemma 11.2. Let us suppose that there exists an element $c \in \mathbb{C}$ such that the equality (11.1) holds; this implies that

$$
D^{1} \varphi^{\prime}=c \tilde{\sigma}\left(d \tilde{f}_{r_{1}, \ldots, r_{n-1}}^{\prime}\right) .
$$

Since the function $\psi$ is everywhere non-vanishing, from the equalities (11.4) and (11.6)-(11.8), we infer that the coefficients $c$ and $a_{1}, \ldots, a_{n-1}$ satisfy the relations (8.3)-(8.5) of [5]. Hence by Proposition 8.1 of [5], we infer that the coefficient $c$ vanishes unless the integers $r_{j}$ and the coefficients $a_{j}$ vanish, for all $2 \leq j \leq n-2$, and the relations

$$
r_{1}=r_{n-1} \geq 1, \quad a_{1}=-a_{n-1}, \quad c=2 i a_{1}
$$

hold. Thus if we consider the element

$$
\beta=\tilde{f}_{n-1} d \tilde{f}_{1}-\tilde{f}_{1} d \tilde{f}_{n-1}
$$

of $C^{\infty}\left(T_{\mathbb{C}}^{*}\right)$, we know that the coefficient $c$ vanishes unless $\varphi$ is a multiple of the 1-form $\left(\tilde{f}_{1} \cdot \tilde{f}_{n-1}\right)^{r} \beta$ and $\vartheta=d\left(\tilde{f}_{1} \cdot \tilde{f}_{n-1}\right)^{r+1}$, where $r=r_{1}-1 \geq 0$.

By (11.4), (11.6) and (11.8), we see that the relations

$$
\begin{aligned}
\kappa^{*}\left(\operatorname{Hess} \tilde{f}_{1}^{\prime}\right)\left(\zeta_{1}, \zeta_{1}\right) & =-4, \quad \kappa^{*}\left(\operatorname{Hess} \tilde{f}_{1}^{\prime}\right)\left(\zeta_{1}, \zeta_{2}\right)=0, \\
\kappa^{*} \tilde{\sigma}\left(d \tilde{f}_{1}^{\prime}\right)\left(\zeta_{1}, \zeta_{1}\right) & =\kappa^{*}\left\langle\eta_{1}+\eta_{2}, d \tilde{f}_{1}^{\prime}\right\rangle=\frac{2 i(n-2)}{n}, \\
\kappa^{*} \tilde{\sigma}\left(d \tilde{f}_{1}^{\prime}\right)\left(\zeta_{1}, \zeta_{2}\right) & =-\kappa^{*}\left\langle\eta_{2}, d \tilde{f}_{1}^{\prime}\right\rangle=\frac{2 i}{n}
\end{aligned}
$$

hold at the point 0 of $\mathbb{R}^{n-1}$.

Lemma 11.3. (i) We have the relations

$$
\begin{aligned}
\text { Hess } \tilde{f}_{1} & =-\frac{2}{n} \tilde{f}_{1} g+2 i \tilde{\sigma}\left(d \tilde{f}_{1}\right), \\
\text { Hess } \tilde{f}_{n-1} & =-\frac{2}{n} \tilde{f}_{n-1} g-2 i \tilde{\sigma}\left(d \tilde{f}_{n-1}\right), \\
D^{1} \beta & =2 i \tilde{\sigma}\left(d\left(\tilde{f}_{1} \cdot \tilde{f}_{n-1}\right)\right) .
\end{aligned}
$$

(ii) For $\gamma=\gamma_{1,0, \ldots, 0,1}^{\prime}$, we have the inclusion

$$
\tilde{\sigma} d C_{\gamma}^{\infty}(X) \subset D^{1} C^{\infty}\left(T_{\mathbb{C}}^{*}\right)
$$


Proof. Since the differential operator Hess is homogeneous and the $G$-module $C_{\varpi_{2}}^{\infty}(X)$ is irreducible, from the decomposition (10.9) we obtain the existence of constants $a, b \in \mathbb{C}$ such that

$$
\text { Hess } f=a f g+b \tilde{\sigma}(d f),
$$

for all $f \in C_{\varpi_{2}}^{\infty}(X)$. Since the complex conjugate $\hat{f}_{1}$ of $\tilde{f}_{1}$ is an element of $C_{\varpi_{2}}^{\infty}(X)$, from the relations (9.1), (9.9) and (11.9) we deduce that $a=-2 / n$ and $b=-2 i$, and so we obtain formula (11.10). As $\tilde{f}_{n-1}$ is an element of $C_{\varpi_{2}}^{\infty}(X)$, we have also verified the identity (11.11). By (1.3), we have

$$
D^{1} \beta=\tilde{f}_{n-1} \text { Hess } \tilde{f}_{1}-\tilde{f}_{1} \operatorname{Hess} \tilde{f}_{n-1} ;
$$

the relation (11.12) is now a direct consequence of (11.10) and (11.11). Next the equality (11.12) implies that the symmetric 2 -form $\tilde{\sigma}\left(d\left(\hat{f}_{1} \cdot \hat{f}_{n-1}\right)\right)$ belongs to $D^{1} C^{\infty}\left(T_{\mathbb{C}}^{*}\right)$. We know that the function $\hat{f}_{1} \cdot \hat{f}_{n-1}$ is a highest weight vector of the irreducible $G$-module $C_{\gamma}^{\infty}(X)$, where $\gamma=\varpi_{2}+\varpi_{2 n-2}$, and so we obtain the inclusion (11.13).

We denote by $I_{n-2}$ the identity matrix of order $n-2$. For $\alpha \in \mathbb{R}$, we consider the element

$$
R_{\alpha}=\left(\begin{array}{cc}
\cos \alpha-\sin \alpha \\
\sin \alpha & \cos \alpha
\end{array}\right)
$$

of $S O(2)$ and the element $R_{\alpha}^{\prime}=\left(R_{\alpha}, I_{n-2}\right)$ of the subgroup $S O(2) \times S O(n-2)$ of $S O(n)$. We know that the element $\phi_{\alpha}=\left(R_{\alpha}^{\prime}, R_{\alpha}^{\prime}\right)$ of $S O(n) \times S O(n)$ belongs to $K=S p(n)$; thus we have $\phi_{\alpha}\left(x_{0}\right)=x_{0}$. We consider the functions $\left\{f_{j k, \alpha}\right\}_{1 \leq j, k \leq 2 n-2}$ on $\mathbb{R}^{n-1}$ determined by

$$
\begin{gathered}
f_{12, \alpha}=\cos ^{2} \alpha \cdot e^{2 i x_{1}}+\sin ^{2} \alpha \cdot e^{2 i x_{2}}, \quad f_{34, \alpha}=\sin ^{2} \alpha \cdot e^{2 i x_{1}}+\cos ^{2} \alpha \cdot e^{2 i x_{2}}, \\
f_{14, \alpha}=f_{32, \alpha}=\cos \alpha \cdot \sin \alpha \cdot\left(e^{2 i x_{1}}-e^{2 i x_{2}}\right), \\
f_{j k, \alpha}=-f_{k j, \alpha}, \quad f_{2 l-1,2 l, \alpha}=e^{2 i x_{l}},
\end{gathered}
$$

for $1 \leq j, k \leq 2 n-2$ and $3 \leq l \leq n-1$, where $\theta=\left(\theta_{1}, \ldots, \theta_{n-1}\right) \in \mathbb{R}^{n-1}$ and $x_{1}, \ldots, x_{n}$ are given by (2.13), and $f_{r s, \alpha}=0$, for $1 \leq r<s \leq 2 n-2$, whenever $(r, s)$ is not equal to one of the pairs

$$
(1,2), \quad(2,3), \quad(1,4), \quad(3,4), \quad(2 l-1,2 l),
$$

with $3 \leq l \leq n-1$. We also consider the function $\check{f}_{\alpha}$ on $\mathbb{R}^{n-1}$ defined by

$$
\check{f}_{\alpha}(\theta)=\left(f_{12, \alpha} f_{34, \alpha}-f_{14, \alpha}^{2}\right)(\theta) \cdot e^{2 i\left(\theta_{n-1}-\theta_{2}\right)},
$$


for $\theta=\left(\theta_{1}, \ldots, \theta_{n-1}\right)$. Clearly the equalities

$$
d f_{12, \alpha}=2 i\left(\cos ^{2} \alpha \cdot d \theta_{1}+\sin ^{2} \alpha \cdot\left(d \theta_{2}-d \theta_{1}\right)\right), \quad d \check{f}_{\alpha}=2 i d \theta_{n-1}
$$

hold at the point $0 \in \mathbb{R}^{n-1}$. We easily verify that

$$
\kappa^{*} \phi_{\alpha}^{*} \phi^{*}\left\langle Z_{j}, Z_{k}\right\rangle=f_{j k, \alpha},
$$

for all $1 \leq j, k \leq n-1$; it follows that

$$
\kappa^{*} \phi_{\alpha}^{*} \tilde{f}_{1}^{\prime}=f_{12, \alpha}, \quad \kappa^{*} \phi_{\alpha}^{*} \tilde{f}_{n-1}^{\prime}=\check{f}_{\alpha},
$$

and hence that

$$
\left(\phi_{\alpha}^{*} \tilde{f}_{1}^{\prime}\right)\left(x_{0}\right)=\left(\phi_{\alpha}^{*} \tilde{f}_{n-1}^{\prime}\right)\left(x_{0}\right)=1 .
$$

Thus according to (9.6), (11.14) and (11.15), the symmetric 2-form

$$
h_{\alpha}=\phi_{\alpha}^{*}\left(d \tilde{f}_{1}^{\prime} \cdot d \tilde{f}_{1}^{\prime}-d \tilde{f}_{n-1}^{\prime} \cdot d \tilde{f}_{n-1}^{\prime}\right)
$$

on $X$ satisfies

$$
\left(\sum_{j=1}^{n-1} h_{\alpha}\left(\zeta_{j}, \zeta_{j}\right)+\sum_{j=1}^{n-2} h_{\alpha}\left(\zeta_{j}, \zeta_{j+1}\right)\right)\left(x_{0}\right)=24 \cos ^{2} \alpha \cdot \sin ^{2} \alpha .
$$

Clearly the function of $\alpha$ appearing on right-hand side of equation (11.17) is non-zero.

Let $r \geq 1$ be a given integer. We consider the function $f=\tilde{f}_{1}^{\prime} \cdot \tilde{f}_{n-1}^{\prime}$, the 1 -form $\beta^{\prime}=\phi^{*} \beta$ and the symmetric 2 -form

$$
h_{\alpha}^{\prime}=\phi_{\alpha}^{*}\left(f^{r-1} d f \cdot \beta^{\prime}\right)
$$

on $X$, with $\alpha \in \mathbb{R}$. Since we have

$$
d f \cdot \beta^{\prime}=\tilde{f}_{n-1}^{\prime 2} d \tilde{f}_{1}^{\prime} \cdot d \tilde{f}_{1}^{\prime}-\tilde{f}_{1}^{\prime 2} d \tilde{f}_{n-1}^{\prime} \cdot d \tilde{f}_{n-1}^{\prime},
$$

by (11.16) we see that the equality

$$
h_{\alpha}^{\prime}=h_{\alpha}
$$

holds at $x_{0}$.

Lemma 11.4. If $r \geq 1$ is a given integer, the symmetric 2 -form $D^{1}\left(\left(\tilde{f}_{1} \cdot \tilde{f}_{n-1}\right)^{r} \beta\right)$ is not a section of $\tilde{\sigma}\left(T_{\mathbb{C}}^{*}\right)$. 
Proof. Suppose that the assertion of the lemma is not true for an integer $r \geq 1$; then $D^{1}\left(f^{r} \beta^{\prime}\right)$ is a section of $\tilde{\sigma}\left(T_{\mathbb{C}}^{*}\right)$. According to formulas (11.12) and (1.3), we see that $f^{r-1} d f \cdot \beta^{\prime}$ is also a section of $\tilde{\sigma}\left(T_{\mathbb{C}}^{*}\right)$. Thus for all $\alpha \in \mathbb{R}$, the symmetric 2 -form $h_{\alpha}^{\prime}$ defined by (11.18) satisfies the relation (9.15). The equalities (11.17) and (11.19) now lead us to a contradiction.

Finally, in order to finish the proof of Lemma 11.2, we suppose that $\varphi$ is a multiple of the 1 -form $\left(\tilde{f}_{1} \cdot \tilde{f}_{n-1}\right)^{r} \beta$ and that $\vartheta=d\left(\tilde{f}_{1} \cdot \tilde{f}_{n-1}\right)^{r+1}$, where $r=r_{1}-1 \geq 0$. When $r \geq 1$, Lemma 11.4 tells us that $\varphi=0$; because the form $\tilde{\sigma}(\vartheta)$ is non-zero, the coefficient $c$ must also vanish in this case, and so we have completed the proof of Lemma 11.2.

In order to prove Proposition 11.1, by formula (1.1) it suffices to show that

$$
D^{1} C^{\infty}\left(T_{\mathbb{C}}^{*}\right) \cap \tilde{\sigma} d C^{\infty}(X)=\tilde{\sigma} d \mathcal{B} .
$$

Since the differential operators $D^{1}$ and $\tilde{\sigma} d$ are homogeneous, according to Proposition 2.1 of [3] and Lemma 11.3,(ii) the preceding equality holds if and only if

$$
D^{1} C_{\gamma}^{\infty}\left(T_{\mathbb{C}}^{*}\right) \cap \tilde{\sigma} d C_{\gamma}^{\infty}(X)=\{0\},
$$

for all $\gamma \in \Gamma$, with $\gamma \neq \gamma_{1,0, \ldots, 0,1}^{\prime}$. We now proceed to verify that (11.20) holds and, in the process, complete the proof of Proposition 11.1.

If $\gamma \in \Gamma$ is equal to 0 or does not belong to $\Gamma_{0}$, we know that

$$
d C_{\gamma}^{\infty}(X)=\{0\}
$$

and so the equality (11.20) holds. Now let $r_{1}, \ldots, r_{n-1} \geq 0$ be given integers, which are not all zero and satisfy $\left(r_{1}, \ldots, r_{n-1}\right) \neq(1,0, \ldots, 0,1)$, and consider the element $\gamma=\gamma_{r_{1}, \ldots, r_{n-1}}^{\prime}$ of $\Gamma_{0}$. Suppose that the equality (11.20) does not hold for this element $\gamma$. Since the function $f=\hat{f}_{r_{1}, \ldots, r_{n-1}}$ is a highest weight vector of the irreducible $G$-module $C_{\gamma}^{\infty}(X)$, the inclusion

$$
\tilde{\sigma} d C_{\gamma}^{\infty}(X) \subset D^{1} C_{\gamma}^{\infty}\left(T_{\mathbb{C}}^{*}\right)
$$

holds, and so there exists an element $\psi$ of $\mathcal{W}_{\gamma}$ such that

$$
D^{1} \psi=\tilde{\sigma}(d f) .
$$


Then the element $\varphi=\bar{\psi}$ of $\mathcal{V}_{\gamma}$ satisfies the relation (11.1), with $c=1$. Lemma 11.2 now leads us to a contradiction. Therefore the equality (11.20) holds for all $\gamma \in \Gamma$, and so we have proved Proposition 11.1.

\section{The RedUCED SPACE OF $S U(2 n) / S p(n)$}

Let $n \geq 3$ be a given integer. The center of $G=S U(2 n)$ is the cyclic subgroup $S$ of order $2 n$ given by

$$
S=\left\{e^{i k \pi / n} I_{2 n} \mid 0 \leq k \leq 2 n-1\right\} .
$$

If $s$ is the involution of $G$ considered in $\S 8$ sending an element $A \in G$ into $J_{n} \bar{A} J_{n}^{-1}$, the subgroup

$$
K_{S}=\left\{A \in G \mid A^{-1} s(A) \in S\right\}
$$

of $G$ contains the subgroup $K=S p(n)$ and we easily verify that

$$
K_{S}=\left\{A \in G \mid A=e^{i k \pi / n} B \text {, with } B \in S p(n) \text { and } k \in \mathbb{Z}\right\} .
$$

According to Corollary 9.3, Chapter VII of [8] (see also [5, §1]), we know that $Y=G / K_{S}$ is a symmetric space of compact type, which is the reduced space of $X=G / K$.

We consider the generator $A_{0}=e^{i \pi / n} I_{2 n}$ of the center $S$; we easily see that $A_{0}^{k}$ belongs to $K$ if and only if $k \equiv 0 \bmod n$. Thus we have

$$
K_{S}=K \cdot S=\bigcup_{0 \leq k \leq n-1} K \cdot A_{0}^{k}
$$

and the isometry $\tau$ of $X$ induced by the action of the element $A_{0}$ of $G$ on $X$ generates a cyclic group $\Sigma$ of isometries of $X$ of order $n$. Clearly the group $\Sigma$ acts on freely on $X$ and its action commutes with the action of $G$. The quotient of $X$ by $\Sigma$ is the symmetric space $Y=G / K_{S}$ and the natural projection $\varpi: X \rightarrow Y$ is a $n$-fold covering; moreover, the action of the group $G$ on $X$ passes to the quotient $Y$.

We consider the $G$-submodule $C^{\infty}(X)^{\Sigma}$ of $C^{\infty}(X)$ consisting of all $\Sigma$-invariant (or equivalently $\tau$-invariant) functions on $X$. The action of $G$ on $Y$ induces a $G$-module structure on the space $C^{\infty}(Y)$, and the projection $\varpi$ induces an isomorphism

$$
\varpi^{*}: C^{\infty}(Y) \rightarrow C^{\infty}(X)^{\Sigma}
$$


of $G$-modules. If $\gamma$ is an element of $\Gamma$, we denote by $C_{\gamma}^{\infty}(Y)$ the isotypic component of the $G$-module $C^{\infty}(Y)$ corresponding to $\gamma$ and we write

$$
C_{\gamma}^{\infty}(X)^{\Sigma}=C^{\infty}(X)^{\Sigma} \cap C_{\gamma}^{\infty}(X) .
$$

Then the isomorphism $\varpi$ induces an isomorphism of $G$-modules

$$
\varpi^{*}: C_{\gamma}^{\infty}(Y) \rightarrow C_{\gamma}^{\infty}(X)^{\Sigma}
$$

For $p \geq 2$, we know that

$$
\tau^{*} \sigma_{p}=\sigma_{p}
$$

Thus the symmetric $p$-form $\sigma_{p}$ induces an $G$-invariant symmetric $p$-form $\sigma_{Y, p}$ on $Y$ such that

$$
\sigma_{p}=\varpi^{*} \sigma_{Y, p}
$$

We shall always consider the symmetric space $Y=G / K_{S}$ endowed with the $G$-invariant Riemannian metric $g_{Y}=\sigma_{Y, 2}$. For $p \geq 3$, we consider the monomorphism of vector bundles

$$
\tilde{\sigma}_{Y, p}: T_{Y}^{*} \rightarrow S^{p-1} T_{Y}^{*}
$$

induced by the symmetric $p$-form $\sigma_{Y, p}$. We write $\sigma_{Y}=\sigma_{Y, 3}$ and $\tilde{\sigma}_{Y}=\tilde{\sigma}_{Y, 3}$. If $\varphi$ is a 1 -form on $Y$, we have

$$
\varpi^{*} \tilde{\sigma}_{Y, p}(\varphi)=\tilde{\sigma}_{p}\left(\varpi^{*} \varphi\right)
$$

According to Lemma 2.1 of [4] and Lemma 9.2, we see that a 1-form $\varphi$ on $Y$ satisfies the Guillemin condition if and only if the symmetric $(p-1)$-form $\tilde{\sigma}_{Y, p}(\varphi)$ on $Y$ satisfies the Guillemin condition.

From the definition of $f_{k}$, we directly see that

$$
\tau^{*} \tilde{f}_{k}=e^{2 i k \pi / n} \tilde{f}_{k},
$$

for all $1 \leq k \leq n-1$. Let $r_{1}, \ldots, r_{n-1} \geq 0$ be given integers and consider the element $\gamma=\gamma_{r_{1}, \ldots, r_{n-1}}^{\prime}$ of $\Gamma_{0}$. According to (12.3), the function $\tilde{f}_{r_{1}, \ldots, r_{n-1}}$ on $X$ is invariant under the isometry $\tau$ if and only if the relation (7.1) holds. Since the complex conjugate $\hat{f}_{r_{1}, \ldots, r_{n-1}}$ of the function $\tilde{f}_{r_{1}, \ldots, r_{n-1}}$ belongs to the irreducible $G$-module $C_{\gamma}^{\infty}(X)$, we infer that $C_{\gamma}^{\infty}(X)$ is a $G$-submodule of $C^{\infty}(X)^{\Sigma}$ if and only if the relation (7.1) holds. For $1 \leq j \leq n-1$, a section $\varphi_{j}$ of $T_{\mathbb{C}}^{*}$ associated with the integers $r_{1}, \ldots, r_{n-1}$ is $\Sigma$-invariant if and only if the integers $r_{1}, \ldots, r_{n-1}$ satisfy the relation (7.1). 
We denote by $\Gamma_{1}$ the subset of $\Gamma_{0}$ consisting of all elements $\gamma_{r_{1}, \ldots, r_{n-1}}^{\prime}$ of $\Gamma_{0}$, where $r_{1}, \ldots, r_{n-1} \geq 0$ are integers satisfying the relation (7.1). Then by Proposition 2.1 of [3], we have the following result:

Lemma 12.1. (i) Let $r_{1}, \ldots, r_{n-1} \geq 0$ be given integers. The function $\tilde{f}_{r_{1}, \ldots, r_{n-1}}$ on the symmetric space $X=G / K$ is induced by a function on the reduced space $Y=G / K_{S}$ of $X$ if and only if the relation (7.1) holds.

(ii) The $G$-module $\bigoplus_{\gamma \in \Gamma_{1}} C_{\gamma}^{\infty}(X)$ is a dense submodule of $C^{\infty}(X)^{\Sigma}$ and the G-module $\bigoplus_{\gamma \in \Gamma_{1}} C_{\gamma}^{\infty}(Y)$ is a dense submodule of $C^{\infty}(Y)$.

We consider the element $\gamma=\gamma_{1,0, \ldots, 0,1}^{\prime}$ of $\Gamma_{1}$; we know that

$$
C_{\gamma}^{\infty}(X)^{\Sigma}=\mathcal{B}
$$

Therefore $\mathcal{B}_{Y}=C_{\gamma}^{\infty}(Y)$ is an irreducible $G$-module isomorphic to $\mathcal{B}$ and is invariant under conjugation; thus $\mathcal{B}_{Y}$ is equal to the complexification of the subspace

$$
\mathcal{B}_{Y, \mathbb{R}}=\left\{f \in \mathcal{B}_{Y} \mid f=\bar{f}\right\}
$$

of $C_{\mathbb{R}}^{\infty}(Y)$ and the mapping $\varpi$ induces an isomorphism $\varpi^{*}: \mathcal{B}_{Y, \mathbb{R}} \rightarrow \mathcal{B}_{\mathbb{R}}$.

If $P$ denotes the orthogonal projection corresponding to the decomposition (1.5) on the space $Y$, according to Lemma 1.1 of [4] and Lemma 9.2 the mapping

$$
P_{\sigma_{Y}}=P \tilde{\sigma}_{Y} d: C_{\mathbb{R}}^{\infty}(Y) \rightarrow I(Y)
$$

is well-defined. We denote by $\mathcal{F}_{Y}$ the orthogonal complement of the finitedimensional space $\mathcal{F}_{Y}^{\prime}=\mathbb{R}(Y) \oplus \mathcal{B}_{Y}$ in $C_{\mathbb{R}}^{\infty}(Y)$. From Proposition 1.2 of [4] and Proposition 11.1, we obtain:

Theorem 12.2. The reduced symmetric space $Y=G / K_{S}$ is not rigid in the sense of Guillemin. If $f$ is a non-zero element of $\mathcal{F}_{Y}$, then the symmetric 2-form $\tilde{\sigma}_{Y}(d f)$ on $Y$ satisfies the Guillemin condition and is not a Lie derivative of the metric. Moreover, the relation

$$
D_{0} C^{\infty}\left(T_{Y}\right) \cap \tilde{\sigma}_{Y} d C_{\mathbb{R}}^{\infty}(Y)=\tilde{\sigma}_{Y} d \mathcal{B}_{Y}
$$

holds and the kernel of the mapping (12.4) is the finite-dimensional space

$$
\mathcal{F}_{Y}^{\prime}=\mathbb{R}(Y) \oplus \mathcal{B}_{Y, \mathbb{R}} .
$$




\section{REFERENCES}

[1] N. Bourbaki, Élements de Mathématique, Livre II, Algèbre, Chapitre 3, Algèbre Multilinéaire, Hermann, Paris, 1958.

[2] W. Fulton and J. Harris, "Representation theory: a first course," Graduate Texts in Math., Vol. 129, Springer-Verlag, New York, Berlin, Heidelberg, 1991.

[3] J. Gasqui and H. Goldschmidt, "Radon transforms and the rigidity of the Grassmannians," Ann. of Math. Studies, No. 156, Princeton University Press, Princeton, NJ, Oxford, 2004.

[4] Infinitesimal isospectral deformations of the Grassmannian of 3-planes in $\mathbb{R}^{6}$, Mém. Soc. Math. Fr. (N.S.), 109 (2007).

[5] _ Infinitesimal isospectral deformations of the Lagrangian Grassmannians, Ann. Inst. Fourier (Grenoble), 57 (2007), 2143-2182.

[6] R. Goodman and N. Wallach, "Representations and invariants of the classical groups," Cambridge University Press, Cambridge, 1998.

[7] V. Guillemin, On micro-local aspects of analysis on compact symmetric spaces, in "Seminar on micro-local analysis," by V. Guillemin, M. Kashiwara and T. Kawai, Ann. of Math. Studies, No. 93, Princeton University Press, University of Tokyo Press, Princeton, NJ, 1979, 79-111.

[8] S. Helgason, "Differential geometry, Lie groups, and symmetric spaces," Academic Press, Orlando, FL, 1978.

[9] T. Vust, Opération de groupes réductifs dans un type de cônes homogènes, Bull. Soc. Math. France, 102 (1974), 317-333.

Jacques Gasqui

Institut Fourier

Université Joseph Fourier, 100 rue des Maths,

BP 116,

38402 Saint-Martin d'Hères, France

E-mail: jacques.gasqui@ujf-grenoble.fr

Hubert Goldschmidt

Department of Mathematics

Columbia University, MC 4406, 2990 Broadway,

New York, NY 10027, USA

E-mail: hg@math.columbia.edu 